\title{
Reductions of Topologically Massive Gravity II: First Order Realizations of Second Order Lagrangians
}

\author{
Filiz Çağatay Uçgun ${ }^{1}$, Oğul Esen ${ }^{2}$ and Hasan Gümral ${ }^{3}$
}

\author{
${ }^{1}$ Department of Software Engineering, Maltepe University, 34857 Maltepe, İstanbul, Turkey, filizcagatayuc- \\ gun@maltepe.edu.tr \\ ${ }^{2}$ Department of Mathematics, Gebze Technical University, 41400 Gebze, Kocaeli, Turkey, oesen@gtu.edu.tr \\ ${ }^{3}$ Department of Mathematics, Yeditepe University, 34755 Ataşehir, İstanbul, Turkey, hgumral@yeditepe.edu.tr
}

Abstract: Second order degenerate Clèment and Sarığlu-Tekin Lagrangians are casted into forms of various first order Lagrangians. Hamiltonian analysis of these equivalent formalisms are performed by means of DiracBergmann constraint algorithm.

Key words: Second order degenerate Lagrangians, the Dirac-Bergmann algorithm, Sarıoglu-Tekin Lagrangian, Clément Lagrangian.

AMS2010: 70H45, 70H50, 70H05, 83E05.

\section{Contents}

1 Introduction $\quad 2$

2 Fundamentals 2

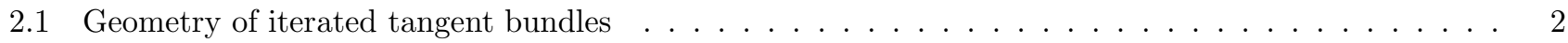

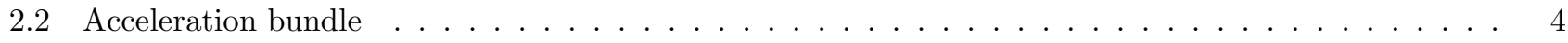

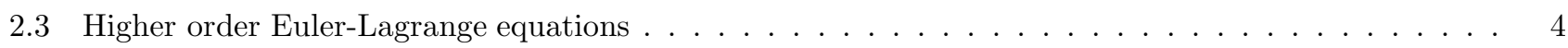

2.4 The Legendre transformation and the Dirac-Bergmann algorithm . . . . . . . . . . . . . . . 5

3 Reduction of the second order Lagrangian theories to the first order ones 6

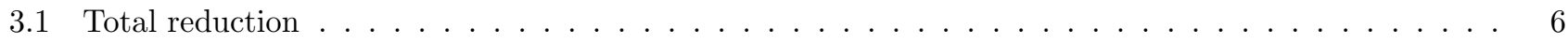

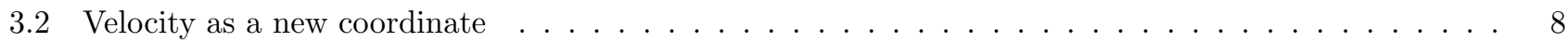

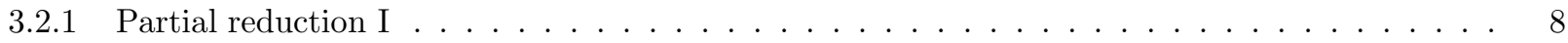

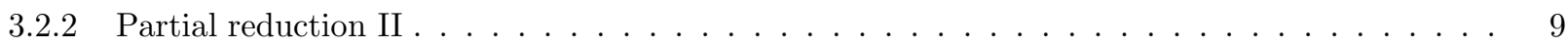

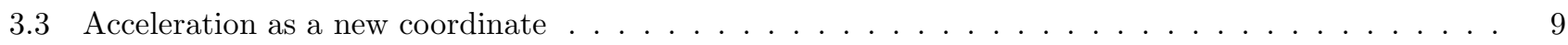

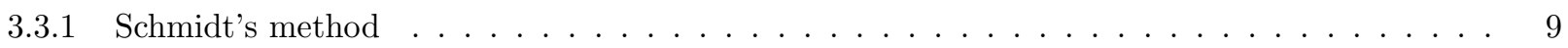

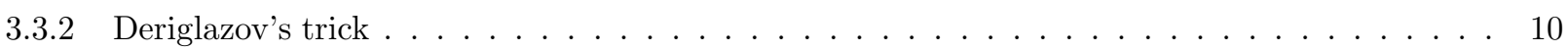

4 Clèment Lagrangian $\quad 10$

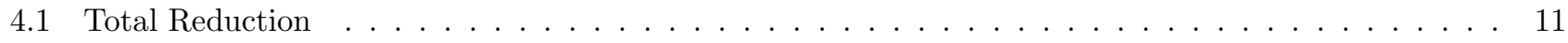

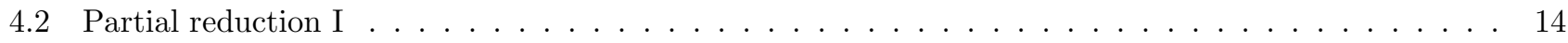

4.2 .1 Unconstrained Variational Formalism . . . . . . . . . . . . . . . . 18

4.3 Partial reduction II . . . . . . . . . . . . . . . . . . . . . . . . . . . . . . . . . . . . 20

4.4 Deriglazov's trick / Schmidt's method . . . . . . . . . . . . . . . . . . . . . 21

5 Sarığlu-Tekin Lagrangian $\quad 24$

5.1 Total Reduction . . . . . . . . . . . . . . . . . . . . . . . . 24

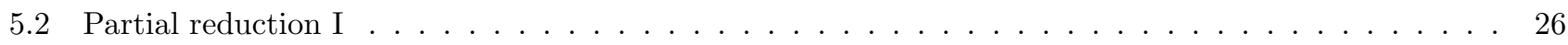

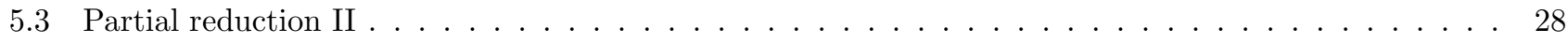

5.4 Deriglazov's trick / Schmidt's method . . . . . . . . . . . . . . . . . . . 30

6 Conclusions 31

7 Acknowledgment $\quad[31$ 


\section{Introduction}

A particularly interesting class of degenerate second order Lagrangians arises in the theory of topologically massive gravity [25, 26], namely Clément, and Sarıŏlu-Tekin Lagrangians. Clément introduced

$$
L^{C}=-\frac{m}{2} \zeta \dot{\mathbf{x}}^{2}-\frac{2 m \Lambda}{\zeta}+\frac{\zeta^{2}}{2 \mu m} \mathbf{x} \cdot(\dot{\mathbf{x}} \times \ddot{\mathbf{x}})
$$

while searching particle like solutions of the topologically massive gravity consisting of actions for cosmological gravity and Chern-Simons term [13, 14, 15. The Clément Lagrangian (1) is depending on positions $\mathbf{x}$, velocities $\dot{\mathbf{x}}$ and accelerations $\ddot{\mathbf{x}}$. Here, the inner product is defined by the Lorentzian metric, $\zeta=\zeta(t)$ is a function which allows arbitrary reparametrization of the variable $t$ whereas $\Lambda$ and $1 / 2 m$ are the cosmological and Einstein gravitational constants, respectively. Sarı̆̆lu-Tekin Lagrangian

$$
L^{S T}=\frac{1}{2}\left[a\left(\dot{\mathbf{x}}^{2}+\dot{\mathbf{y}}^{2}\right)+\frac{2}{\mu} \dot{\mathbf{y}} \cdot \ddot{\mathbf{x}}-m^{2}\left(\mathbf{y}^{2}+\mathbf{x}^{2}\right)\right],
$$

proposed in [64] while studying actions consisting of Einstein-Hilbert, Chern-Simons and Pauli-Fierz terms. Here, $a, \mu, m$ are scalars, and $\mathbf{x}, \mathbf{y}$ are the position three-vectors.

The Legendre transformations of higher order Lagrangian formalisms can be achieved by defining Ostrogradski momenta [56. If the Lagrangian is non-degenerate in the sense of Ostrogradski, then the Legendre transformation is immediate. If it is degenerate, then one additionally needs to employ the Dirac-Bergmann algorithm [6, 27, 28, 72 , to arrive at a proper submanifold of momentum phase space in order to have a well-defined Hamilton's equations.

In our previous work [8], we obtained Hamiltonian formalisms of both the Clément Lagrangian (11) and the Sarığlu-Tekin Lagrangian (2) by writing Ostrogradski momenta, and applying Dirac analysis on associated momentum phase spaces. In the present paper, we shall continue to the Hamiltonian analysis of Clément (1) and Sarığlu-Tekin (2) Lagrangians by recasting the second order Lagrangians into first order forms on some iterated tangent bundles. Such reduction procedures are far from being unique and are mainly achieved by enlarging the configuration space with introductions of new coordinates and Lagrange multipliers. In reduction procedures, the reduced first order Lagrangian would be degenerate because of presence of the Lagrange multipliers even if the second order one is not. Hence, in this work, while presenting the Hamiltonian formalizations of the Clément and Sarığlu-Tekin Lagrangians, we shall be facing with two kinds of degeneracies. The first one is due to the functional structures of the Lagrangians, that is degeneracies in the sense of Ostrogradski. The second one is due to the degeneracies evolving in the reduction procedures.

This paper is organized into four main sections. In the following section, some preliminary aspects, such as the higher order Euler-Lagrange equations, the Legendre transformation, and the Dirac-Bergmann algorithm, will be exhibited for the sake of completeness. In the third section, we shall summarize three possible ways to recast a second order Lagrangian function into a first order form. In total reduction, this is achieved by considering both velocity and acceleration variables as independent coordinates. In this case, Lagrange multipliers will turn out to be Ostrogradski's momenta. In the second way, that we call partial reductions, only velocity variable is defined to be an independent variable. In the third way, only acceleration variable is considered as a new coordinate. This last kind of reduction considered in different contexts as the Schmidt method [2, 67, or the Deriglazov's trick [23. We shall prove that these two methods are the same for second order theories in the realm of acceleration bundle 29 . Novelty of this work can be found in the the fourth, and the fifth sections which are reserved for applications of techniques presented in the third section to the Clément (11) and the Sarığlu-Tekin (2) Lagrangians, respectively. For each case, reduced Lagrangian functions, and Legendre transformations are explicitly presented. Accordingly, total Hamiltonian functions, applications of the Dirac-Bergmann algorithm to presymplectic structures, and Dirac brackets are exhibited.

\section{Fundamentals}

\subsection{Geometry of iterated tangent bundles}

Let $Q$ be a manifold. Consider the set $C_{q}(Q)$ of smooth curves passing through a point $q$ in $Q$. Two curves $\gamma$ and $\gamma^{\prime}$ are called $k$-equivalent and denoted by $\gamma \sim_{q}^{k} \gamma^{\prime}$, if they agree up to their $k$-th order derivatives at $q$ that is if

$$
D^{r}(f \circ \gamma)(0)=D^{r}\left(f \circ \gamma^{\prime}\right)(0), \quad r=0,1,2, \ldots, k,
$$


for all real valued functions $f$ defined on $Q$ [47. Here, we assumed that $\gamma(0)=\gamma^{\prime}(0)=q$. Under the equivalence relationship $\sim_{q}^{k}$, an equivalence class, denoted by $\mathfrak{t}^{k} \gamma(0)$, is called a $k$-th order tangent vector at $q$. The set of all equivalence classes of curves, that is the set of all $k$-th order tangent vectors at $q$ defines $k$-th order tangent space $T_{q}^{k} Q$. The union

$$
T^{k} Q=\bigsqcup_{q \in Q} T_{q}^{k} Q
$$

of all $k$-th order tangent spaces is $k$-th order tangent bundle of $Q$.

There exists a projection from the $k$-th order tangent bundle $T^{k} Q$ to the manifold $Q$ defined as

$$
\tau_{Q}^{k}: T^{k} Q \longrightarrow Q: \mathfrak{t}^{k} \gamma(0) \longrightarrow \gamma(0) .
$$

All possible triples

$$
\left(T^{k} Q, \tau_{Q}^{k}, Q\right)
$$

are fiber bundles with total space $T^{k} Q$, the projection $\tau_{Q}^{k}$, and the base space $Q$. If $k=1$ then we arrive at the tangent bundle $\left(T Q, \tau_{Q}, Q\right)$. $T Q$ admits a vector bundle structure on $Q$ but in general $T^{k} Q$ does not necessarily admit a vector bundle structure on $Q$ for $k \geq 2$ [73].

Assume that $Q$ is an $n$-dimensional manifold with local coordinates $(\mathbf{q})=\left(q^{1}, \ldots, q^{n}\right)$, then the first order tangent bundle $T Q$ is a $2 n$-dimensional manifold with induced coordinates

$$
(\mathbf{q}, \dot{\mathbf{q}}): T Q \longrightarrow \mathbb{R}^{2 n}: \mathfrak{t} \gamma(0) \longrightarrow(\mathbf{q} \circ \gamma(0), D(\mathbf{q} \circ \gamma)(0)) .
$$

The $k$-th order tangent bundle $T^{k} Q$ is a $[(k+1) n]$-dimensional manifold with induced coordinates

$$
\left(\mathbf{q}, \dot{\mathbf{q}}, \ldots, \mathbf{q}^{(k)}\right): T^{k} Q \longrightarrow \mathbb{R}^{(k+1) n}: \mathfrak{t}^{k} \gamma(0) \longrightarrow\left(\mathbf{q} \circ \gamma(0), D(\mathbf{q} \circ \gamma)(0), \ldots, D^{k}(\mathbf{q} \circ \gamma)\right) .
$$

There are two possible ways to write the iterated bundle $T T Q$ as a vector bundle over the tangent bundle $T Q$. The geometry of this double vector bundle structure [37. can be summarized in the following commutative diagram, called as tangent rhombic,

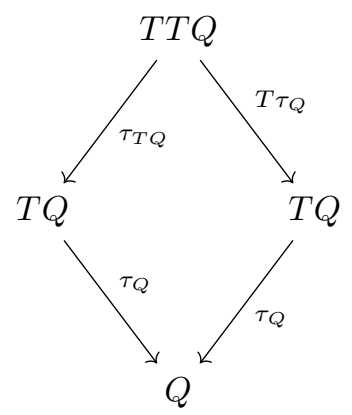

where $\tau_{T Q}$ is the tangent bundle projection whereas $T \tau_{Q}$ is the tangent lift of $\tau_{Q}$. In terms of a local coordinate system $(\mathbf{q}, \mathbf{v}, \dot{\mathbf{q}}, \dot{\mathbf{v}})$ on $T T Q$, these projections are computed to be

$$
\begin{aligned}
\tau_{T Q}: T T Q \longrightarrow T Q:(\mathbf{q}, \mathbf{v}, \dot{\mathbf{q}}, \dot{\mathbf{v}}) & \mapsto(\mathbf{q}, \mathbf{v}), \\
T \tau_{Q}: T T Q \longrightarrow T Q: & (\mathbf{q}, \mathbf{v}, \dot{\mathbf{q}}, \dot{\mathbf{v}}) \mapsto(\mathbf{q}, \dot{\mathbf{q}}) .
\end{aligned}
$$

In terms of these projections, we present the embedding of the second order tangent bundle $T^{2} Q$ into $T T Q$ as follows

$$
T^{2} Q=\left\{\mathbf{z} \in T T Q: \tau_{T Q}(\mathbf{z})=T \tau_{Q}(\mathbf{z})\right\}
$$

which, in a local frame $(\mathbf{q}, \dot{\mathbf{q}}, \mathbf{v}, \dot{\mathbf{v}})$ on $T T Q$, reads

$$
T^{2} Q=\{(\mathbf{q}, \mathbf{v}, \dot{\mathbf{q}}, \dot{\mathbf{v}}) \in T T Q: \dot{\mathbf{q}}-\mathbf{v}=\mathbf{0}\} .
$$

Existence of this embedding leads us to write a second order Lagrangian defined on $T^{2} Q$ as a first order Lagrangian function defined on $T T Q$ along with the constraint $\dot{\mathbf{q}}-\mathbf{v}=\mathbf{0}$. Such a first order realization of a second order Lagrangian function fails to be unique due to the existence of the double vector bundle structure of $T T Q$ pictured in (6). We discuss this duality in the framework of Lagrangian dynamics by labeling them as partial reduction I and partial reduction II in Subsections (3.2.1) and (3.2.2), respectively. They will provide two possible ways to define velocity as a new coordinate in a second order framework. 


\subsection{Acceleration bundle}

It is interesting to note a submanifold of $T T Q$ defined by

$$
A Q=\left\{\mathbf{z} \in T T Q: \tau_{T Q}(\mathbf{z})=T \tau_{Q}(\mathbf{z})=\mathbf{0}\right\}
$$

We call $A Q$ as acceleration bundle [29. Comparing the definitions of $T^{2} Q$ and $A Q$ given in (9) and (11), respectively, one immediately observes that $A Q$ is also a submanifold of $T^{2} Q$. Accordingly, $A Q$ can be identified with the intersection

$$
A Q=V T Q \cap T^{2} Q
$$

where $V T Q$ is the vertical bundle consisting of vectors on $T Q$ projecting to the zero vectors on $Q$ via the mapping $T \tau_{Q}$.

Here is a direct way to define $A Q$ without referring to $T T Q$. Consider the set $C_{q}(Q)$ of smooth curves passing through the point $q$ in $Q$. We define a subset $K_{q}(Q)$ of $C_{q}(Q)$ by considering only the curves whose first derivatives are vanishing at $q$. More formally,

$$
K_{q}(Q)=\left\{\gamma \in C_{q}(Q): D(f \circ \gamma)(0)=0, \quad \forall f: Q \mapsto \mathbb{R}\right\}
$$

It is worthless to say that since vanishing of the first derivative is asked only at a single point, the curve $\gamma$ in $K_{q}(Q)$ does not have to be a constant curve. We are now defining an equivalence relation on $K_{q}(Q)$. We call two curves $\gamma$ and $\gamma^{\prime}$ in $K_{q}(Q)$ equivalent if the second derivatives of $\gamma$ and $\gamma^{\prime}$ are equal at $q$, that is if

$$
D^{2}(f \circ \gamma)(0)=D^{2}\left(f \circ \gamma^{\prime}\right)(0)
$$

for all real valued functions $f$ on $Q$. Here, it is assumed that $\gamma(0)=\gamma^{\prime}(0)=q$. An equivalence class is denoted by $\mathfrak{a} \gamma(0)$. The set of all equivalence classes in $K_{q}(Q)$ is called acceleration space $A_{q} Q$ at $q \in Q$. If $Q$ is an $n$-dimensional manifold with local coordinates $(\mathbf{q})$, then union of all acceleration spaces

$$
A Q=\bigsqcup_{q \in Q} A_{q} Q
$$

is a $2 n$-dimensional manifold with induced local coordinates

$$
(\mathbf{q}, \mathbf{a}): A Q \longrightarrow \mathbb{R}^{2 n}: \mathfrak{a} \gamma(0) \longrightarrow\left(\mathbf{q} \circ \gamma(0), D^{2}(\mathbf{q} \circ \gamma)(0)\right)
$$

Assume that $(\mathbf{q})$ and $(\mathbf{x})$ be two compatible charts around a point $q$ in $Q$. Then, the induced local charts on $A Q$, given by $(\mathbf{q}, \mathbf{a})$ and $(\mathbf{x}, \mathbf{b})$, are also compatible. Transformations relating these two local pictures are computed to be

$$
\mathbf{x}=\mathbf{x}(\mathbf{q}), \quad \mathbf{b}=\left(\mathbf{a} \cdot \frac{\partial}{\partial \mathbf{q}}\right) \mathbf{x}
$$

These coordinate transformations suggest a vector bundle structure of $A Q$ over $Q$ with projection

$$
\alpha_{Q}: A Q \rightarrow Q: \mathfrak{a} \gamma(0) \longrightarrow \gamma(0)
$$

Acceleration bundle geometry will lead us to introduce an alternative reduction procedure to write a second order Lagrangian function as a first order Lagrangian function by labeling the acceleration as a new coordinate instead of the velocity. Accordingly, in Subsection (3.3), we shall elaborate the geometries of both Schmidt's method and Deriglazov's trick in the realm of $A Q$.

\subsection{Higher order Euler-Lagrange equations}

A Lagrangian function $L$ is a real valued function on $T Q$, and generates the Euler-Lagrange equations

$$
\frac{\partial L}{\partial \mathbf{q}}-\frac{d}{d t} \frac{\partial L}{\partial \dot{\mathbf{q}}}=\mathbf{0} .
$$


Second order tangent bundle $T^{2} Q$ of $Q$ is $3 n$-dimensional manifold with local coordinates $(\mathbf{q} ; \dot{\mathbf{q}} ; \ddot{\mathbf{q}})$. A second order Lagrangian function $L=L(\mathbf{q} ; \dot{\mathbf{q}} ; \ddot{\mathbf{q}})$ is a real-valued function on $T^{2} Q$. The second order Euler-Lagrange equations are

$$
\frac{\partial L}{\partial \mathbf{q}}-\frac{d}{d t} \frac{\partial L}{\partial \dot{\mathbf{q}}}+\frac{d^{2}}{d t^{2}} \frac{\partial L}{\partial \ddot{\mathbf{q}}}=\mathbf{0}
$$

Notice that, the set (18) of second order Euler-Lagrange equations consists of the fourth order differential equations only if one of the components of $\partial L / \partial \ddot{\mathbf{q}}$ depends on the acceleration $\ddot{\mathbf{q}}$. While presenting Schmidt's method in Subsection (3.3.1), we shall need the Euler-Lagrange equations for the third order Lagrangians. Accordingly, we record here the third order Euler-Lagrange equations

$$
\frac{\partial L}{\partial \mathbf{q}}-\frac{d}{d t} \frac{\partial L}{\partial \dot{\mathbf{q}}}+\frac{d^{2}}{d t^{2}} \frac{\partial L}{\partial \ddot{\mathbf{q}}}-\frac{d^{3}}{d t^{3}} \frac{\partial L}{\partial \mathbf{q}^{(3)}}=\mathbf{0} .
$$

generated by a third order Lagrangian function $L=L\left(\mathbf{q} ; \dot{\mathbf{q}} ; \ddot{\mathbf{q}} ; \mathbf{q}^{(3)}\right)$.

\subsection{The Legendre transformation and the Dirac-Bergmann algorithm}

Hamiltonian representation of a physical system is defined on the cotangent bundle $T^{*} Q$ of the configuration manifold $Q$ [1]. Physically, $T^{*} Q$ corresponds to the momentum-phase space of a physical system. $T^{*} Q$ carries a canonical symplectic two-form $\Omega_{Q}$, hence a non-degenerate Poisson structure $\{\bullet, \bullet\}$. Dynamics of an observable $f$ governed by a Hamiltonian function $H$ is determined by the Hamilton's equations

$$
\dot{f}=\{f, H\} .
$$

If we equip $T^{*} Q$ with the Darboux' coordinates $(\mathbf{q}, \mathbf{p})$ then the canonical Poisson bracket relations can be determined by

$$
\{\mathbf{p}, \mathbf{q}\}=\mathbb{I},
$$

and the rest of all possible brackets are identically zero. Here, $\mathbb{I}$ is the identity matrix. In this coordinate frame, the Hamilton's equations turn out to be

$$
\dot{\mathbf{q}}=\frac{\partial H}{\partial \mathbf{p}}, \quad \dot{\mathbf{p}}=-\frac{\partial H}{\partial \mathbf{q}} .
$$

For Hamiltonian formulation of the first order Euler-Lagrange equations (17), one needs to define a passage from the tangent bundle $T Q$ to the cotangent bundle $T^{*} Q$. This is achieved by means of the fiber derivative of a Lagrangian function $L$ that is

$$
\mathbb{F} L: T Q \rightarrow T^{*} Q:(\mathbf{q}, \dot{\mathbf{q}}) \rightarrow\left(\mathbf{q}, \frac{\partial L}{\partial \dot{\mathbf{q}}}\right) .
$$

It is evident that, in order to make the transformation (22) invertible, one needs to employ a non-degeneracy condition, called the Hessian condition,

$$
\operatorname{det} \frac{\partial^{2} L}{\partial \dot{\mathbf{q}}^{2}} \neq 0
$$

In this case, the velocities $\dot{\mathbf{q}}$ can uniquely be written as functions of position and momenta, and the Hamiltonian function on $T^{*} Q$ is defined to be

$$
H(\mathbf{q}, \mathbf{p})=\mathbf{p} \cdot \dot{\mathbf{q}}(\mathbf{q}, \mathbf{p})-L(\mathbf{q}, \dot{\mathbf{q}}(\mathbf{q}, \mathbf{p})) .
$$

Notice that, the Hamilton's equations (21) generated by the Hamiltonian function presented in (24) equal to the first order Euler-Lagrange equations (17).

If the Hessian condition (23) is not satisfied then one cannot solve the velocities $\dot{\mathbf{q}}$ in terms of momenta p. Instead, one arrives at an immersed submanifold, called primary constraint submanifold, $C$ of $T^{*} Q$. We are assuming that $C$ can be defined as a set of constraint functions, called primary constraints, $\Phi_{a} \approx 0$ on $T^{*} Q$. Here $a$ is ranging from 1 to the codimension $r$ of $C$. The equalities in the primary constraints are weak in the sense that they will be ignored during set up of Dirac formalism, and will actually vanish in any solutions to equations of motion. The dynamics on primary constraint submanifold is not well-defined by the canonical Hamiltonian function (24), it is rather governed by the total Hamiltonian

$$
H_{T}=H+u^{a} \Phi_{a}
$$


which contains linear combinations of the primary constraints with Lagrange multipliers $u^{a}$. The requirement that the solutions of Euler-Lagrange equations remain on the constraint submanifold is described by the weak equality

$$
\dot{\Phi}_{b}=\left\{\Phi_{b}, H_{T}\right\}=\left\{\Phi_{b}, H\right\}+u^{a}\left\{\Phi_{b}, \Phi_{a}\right\} \approx 0 .
$$

These consistency conditions may lead to determination of Lagrange multipliers if the left hand sides contain $u^{a}$. In this case, one solves for $u^{a}$ through the set of linear equations

$$
\left\{\Phi_{b}, \Phi_{a}\right\} u^{a}=-\left\{\Phi_{b}, H\right\}
$$

for which the solution set, namely, number of multipliers that can be solved is characterized by the rank of the skew-symmetric matrix $\left\{\Phi_{b}, \Phi_{a}\right\}$ of Poisson brackets. Obviously, if the number of primary constraints is odd then Lagrange multipliers cannot be solved completely and one aspects more constraints to determine $H_{T}$ in terms of phase space variables. This secondary constraints follow if the left hand sides does not contain $u^{a}$ or, $n-r$ is odd. Repeating this process, one enlarges the primary constraint set with the new (secondary, tertiary, etc.) constraints, redefines $H_{T}$ by introducing new Lagrange multipliers for new constraints and, repeats the consistency computations. Iterated applications of consistency computations lead to a complete set of constraints $\Phi_{\alpha}: \alpha=1, \ldots, k$. Let

$$
\mathcal{M}_{\alpha \beta}=\left\{\Phi_{\alpha}, \Phi_{\beta}\right\}
$$

be the matrix of Poisson brackets of constraints modulo all constraints. If $\operatorname{rank}\left(M_{\alpha \beta}\right)=s$, then $\operatorname{ker}\left(M_{\alpha \beta}\right)$ is $(k-s)$-dimensional. A basis for the kernel can be constructed from linear combinations $\psi_{\alpha}$ of $\Phi_{\alpha}$ satisfying

$$
\left\{\psi_{\alpha}, \psi_{\beta}\right\} \approx 0, \quad \alpha, \beta=1, \ldots, k-s
$$

and are called as the first class constraints. Note that the number of Lagrange multipliers which can be solved is also determined by the matrix of all constraints. Let $\chi_{\alpha}: \alpha=1, \ldots, s$ be the second class constraints whose Poisson brackets does not vanish (modulo constraints). Define the $s \times s-$ matrix

$$
C_{\alpha \beta}=\left\{\chi_{\alpha}, \chi_{\beta}\right\}, \quad \alpha, \beta=1, \ldots, s
$$

which is invertible by construction. Define the Dirac bracket

$$
\{f, g\}_{D B}=\{f, g\}-\left\{f, \chi_{\alpha}\right\}\left(C^{-1}\right)^{\alpha \beta}\left\{\chi_{\beta}, g\right\}
$$

[72. Note that, since $\left\{f, \chi_{\alpha}\right\}_{D B}=0$ for arbitrary function $f$, second class constraints can be set to zero either before or after evaluation of Dirac bracket. The initial $2 n$ dimensional Hamiltonian system with $(k-s)$ number of first class and $s$ number of second class constraints reduces to $2 n-2(k-s)-s=2 n-2 k+s$ dimensional

Hamiltonian system equipped with the Dirac bracket and with the total Hamiltonian function. The final bracket eliminates the second class constraints from the set of all constraints leaving a complete set of first class constraints. First class constraints form a closed local symmetry algebra for the system. Computing

$$
\left\{\psi_{\alpha}, H\right\}=c_{\alpha}^{\beta} \psi_{\beta}, \quad\left\{\psi_{\alpha}, \psi_{\beta}\right\}=c_{\alpha \beta}^{\gamma} \psi_{\gamma}
$$

one finds the structure constants of this algebra [27, 28].

\section{Reduction of the second order Lagrangian theories to the first order ones}

\subsection{Total reduction}

Given a second order Lagrangian function

$$
L=L(\mathbf{q} ; \dot{\mathbf{q}} ; \ddot{\mathbf{q}})
$$

on the second order tangent bundle $T^{2} Q$, define the $3 n$-dimensional configuration space $M=T^{2} Q$ with coordinates

$$
\mathbf{q}_{(1)}=\mathbf{q}, \quad \mathbf{q}_{(2)}=\dot{\mathbf{q}}, \quad \mathbf{q}_{(3)}=\ddot{\mathbf{q}},
$$


[36, 54. In order not to forget the constitutional relationships in the coordinates of $M$, we impose the sets of constraints

$$
\dot{\mathbf{q}}_{(1)}-\mathbf{q}_{(2)}=0, \quad \dot{\mathbf{q}}_{(2)}-\mathbf{q}_{(3)}=0
$$

on the tangent bundle $T M$ equipped with coordinates

$$
\left(\mathbf{q}_{(1)}, \mathbf{q}_{(2)}, \mathbf{q}_{(3)} ; \dot{\mathbf{q}}_{(1)}, \dot{\mathbf{q}}_{(2)}, \dot{\mathbf{q}}_{(3)}\right) \in T M
$$

We define the first order Lagrangian function

$$
L_{C}=L\left(\mathbf{q}_{(1)}, \mathbf{q}_{(2)}, \mathbf{q}_{(3)}\right)+\lambda \cdot\left(\dot{\mathbf{q}}_{(1)}-\mathbf{q}_{(2)}\right)+\beta \cdot\left(\dot{\mathbf{q}}_{(2)}-\mathbf{q}_{(3)}\right),
$$

where $\lambda \in \mathbb{R}^{n}$ and $\beta \in \mathbb{R}^{n}$ are Lagrange multipliers. We consider $L_{C}$ as a Lagrangian function on the extented space $T\left(M \times \mathbb{R}^{n} \times \mathbb{R}^{n}\right)$ by letting $\lambda$ and $\beta$ be variables as well. For the first order Lagrangian function $L_{C}$ in (31), the first order Euler-Lagrange equations (17) obtained by variations of $\mathbf{q}_{(1)}, \mathbf{q}_{(2)}, \mathbf{q}_{(3)}, \lambda$ and $\beta$ are

$$
\frac{\partial L}{\partial \mathbf{q}_{(1)}}-\dot{\lambda}=\mathbf{0}, \quad \frac{\partial L}{\partial \mathbf{q}_{(2)}}-\lambda-\dot{\beta}=\mathbf{0}, \quad \frac{\partial L}{\partial \mathbf{q}_{(3)}}-\beta=\mathbf{0}, \quad \dot{\mathbf{q}}_{(1)}-\mathbf{q}_{(2)}=\mathbf{0}, \quad \dot{\mathbf{q}}_{(2)}-\mathbf{q}_{(3)}=\mathbf{0} .
$$

Here, the last two equations are the constraints presented in (30) whereas the second and third equations define the Lagrange multipliers

$$
\lambda=\frac{\partial L}{\partial \mathbf{q}_{(2)}}-\frac{d}{d t} \frac{\partial L}{\partial \mathbf{q}_{(3)}}, \quad \beta=\frac{\partial L}{\partial \mathbf{q}_{(3)}} .
$$

This shows that, the Lagrange multipliers $\lambda$ and $\beta$ cannot be indepedent variables, instead they are determined by the Lagrangian function $L$. By substituting the definitions of $\lambda$ and $\beta$ into the constraint Lagrangian $L_{C}$ in (31) we arrive at a Lagrangian function

$$
L_{U}=L\left(\mathbf{q}_{(1)}, \mathbf{q}_{(2)}, \mathbf{q}_{(3)}\right)+\left(\frac{\partial L}{\partial \mathbf{q}_{(2)}}-\frac{d}{d t} \frac{\partial L}{\partial \mathbf{q}_{(3)}}\right) \cdot\left(\dot{\mathbf{q}}_{(1)}-\mathbf{q}_{(2)}\right)+\left(\frac{\partial L}{\partial \mathbf{q}_{(3)}}\right) \cdot\left(\dot{\mathbf{q}}_{(2)}-\mathbf{q}_{(3)}\right)
$$

defined precisely on the tangent bundle $T M$. We shall call $L_{U}$ as unconstraint Lagrangian function. Note that, by pulling the constraints in (32) back to $T^{2} Q$ we arrive at that

$$
\lambda=\frac{\partial L}{\partial \dot{\mathbf{q}}}-\frac{d}{d t} \frac{\partial L}{\partial \ddot{\mathbf{q}}} \quad \text { and } \quad \beta=\frac{\partial L}{\partial \ddot{\mathbf{q}}} .
$$

It is a matter of direct calculation to show that the first order Euler-Lagrange dynamics generated by both the constraint $L_{C}$ and the unconstraint $L_{U}$ Lagrangian functions coincide with the second order Euler-Lagrange equation (18).

For the Hamilton formalism, from the definitions of conjugate momenta for the first order Lagrangian (31), the primary constraints are defined as

$$
\boldsymbol{\Phi}^{(1)}=\mathbf{p}^{(1)}-\boldsymbol{\lambda}, \quad \boldsymbol{\Phi}^{(2)}=\mathbf{p}^{(2)}-\boldsymbol{\beta}, \quad \boldsymbol{\Phi}^{(3)}=\mathbf{p}^{(3)}, \quad \boldsymbol{\Phi}^{(\lambda)}=\mathbf{p}^{(\lambda)}, \quad \boldsymbol{\Phi}^{(\beta)}=\mathbf{p}^{(\beta)} .
$$

In this case, the total Hamiltonian function becomes

$$
H_{T}=H+\boldsymbol{\Phi}^{(1)} \cdot \mathbf{u}_{(1)}+\boldsymbol{\Phi}^{(2)} \cdot \mathbf{u}_{(2)}+\boldsymbol{\Phi}^{(3)} \cdot \mathbf{u}_{(3)}+\boldsymbol{\Phi}^{(\lambda)} \cdot \mathbf{u}_{\lambda}+\boldsymbol{\Phi}^{(\beta)} \cdot \mathbf{u}_{\beta}
$$

where the canonical Hamiltonian is

$$
H=-L\left(\mathbf{q}_{(1)}, \mathbf{q}_{(2)}, \mathbf{q}_{(3)}\right)+\boldsymbol{\lambda} \cdot \mathbf{q}_{(2)}+\boldsymbol{\beta} \cdot \mathbf{q}_{(3)} .
$$

Note that, the Hamilton function for the present case is only depending on $\mathbf{q}_{(1)}, \mathbf{q}_{(2)}, \mathbf{q}_{(3)}, \boldsymbol{\lambda}$ and $\boldsymbol{\beta}$. 


\subsection{Velocity as a new coordinate}

\subsubsection{Partial reduction I}

Define a $2 n$-dimensional configuration space $N=T Q$ with coordinates

$$
\mathbf{q}_{(1)}=\mathbf{q}, \quad \mathbf{q}_{(2)}=\dot{\mathbf{q}} .
$$

We are imposing the set of constraints $\dot{\mathbf{q}}_{(1)}-\mathbf{q}_{(2)}=0$ in the local frame

$$
\left(\mathbf{q}_{(1)}, \mathbf{q}_{(2)} ; \dot{\mathbf{q}}_{(1)}, \dot{\mathbf{q}}_{(2)}\right) \in T N .
$$

$T N$ can be identified with $4 n$-dimensional iterated tangent bundle $T T Q$. An element of $T N=T T Q$ satisfying the constraint $\dot{\mathbf{q}}_{(1)}-\mathbf{q}_{(2)}=0$ is called a second order vector field [1]. Compare this with the embedding in (9). Define a Lagrangian function

$$
L_{C_{1}}=L\left(\mathbf{q}_{(1)}, \mathbf{q}_{(2)}, \dot{\mathbf{q}}_{(2)}\right)+\boldsymbol{\lambda}_{1} \cdot\left(\dot{\mathbf{q}}_{(1)}-\mathbf{q}_{(2)}\right)
$$

on the space $T\left(T Q \times \mathbb{R}^{n}\right)$ with $\boldsymbol{\lambda}_{1}$ being Lagrange multipliers. The first order Euler-Lagrange equations (17) for $L_{C_{1}}$ turn out to be

$$
\frac{\partial L}{\partial \mathbf{q}_{(1)}}-\dot{\boldsymbol{\lambda}}_{1}=\mathbf{0}, \quad \frac{\partial L}{\partial \mathbf{q}_{(2)}}-\frac{d}{d t} \frac{\partial L}{\partial \dot{\mathbf{q}}_{(2)}}=\boldsymbol{\lambda}_{1}, \quad \dot{\mathbf{q}}_{(1)}-\mathbf{q}_{(2)}=\mathbf{0} .
$$

A direct calculation proves that the system (37) is equivalent to the second order Euler-Lagrange equations (18).

Let $T^{*}\left(T Q \times \mathbb{R}^{n}\right)$ be endowed with coordinates $\left(\mathbf{q}_{(1)}, \mathbf{q}_{(2)}, \boldsymbol{\lambda}_{1} ; \mathbf{p}^{(1)}, \mathbf{p}^{(2)}, \mathbf{p}^{\lambda_{1}}\right)$ and define the conjugate momenta as fiber derivatives of $L_{C_{1}}$. This results in a set of primary constraints

$$
\boldsymbol{\Phi}^{(1)}=\mathbf{p}^{(1)}-\boldsymbol{\lambda}_{1} \approx \mathbf{0}, \quad \boldsymbol{\Phi}^{(2)}=\mathbf{p}^{(2)}-\frac{\partial L}{\partial \dot{\mathbf{q}}_{2}} \approx \mathbf{0}, \quad \boldsymbol{\Phi}^{\left(\lambda_{1}\right)}=\mathbf{p}^{\lambda_{1}} \approx \mathbf{0} .
$$

In this case, the canonical Hamiltonian is computed to be

$$
H_{C_{1}}=\mathbf{p}^{(i)} \cdot \dot{\mathbf{q}}_{(i)}+\dot{\lambda}_{1} \cdot \mathbf{p}^{\lambda_{1}}-L_{C_{1}}=\mathbf{p}^{(i)} \cdot \dot{\mathbf{q}}_{(i)}-L
$$

where $i$ runs from 1 to 2. We further define the total Hamiltonian by adding the constraints in (38) as follows

$$
H_{T_{1}}=H_{C_{1}}+\mathbf{u}_{(\alpha)} \cdot \Phi^{(\alpha)}
$$

where $\alpha$ takes values $\left\{1,2, \boldsymbol{\lambda}_{1}\right\}$ and $\mathbf{u}_{(\alpha)}$ s are the Lagrange multipliers to be defined later. The consistency checks for two sets of constraints under the Hamiltonian dynamics generated by $H_{T_{1}}$ result in

$$
\left\{H_{T_{1}}, \boldsymbol{\Phi}^{(1)}\right\}=-\frac{\partial H_{C_{1}}}{\partial \mathbf{q}_{(1)}}-\mathbf{u}_{\left(\lambda_{1}\right)} \approx 0, \quad\left\{H_{T_{1}}, \boldsymbol{\Phi}^{\left(\lambda_{1}\right)}\right\}=\mathbf{u}_{(1)} \approx 0 .
$$

These equations determine $\mathbf{u}_{\left(\lambda_{1}\right)}$ and $\mathbf{u}_{(1)}$ explicitly. To determine the Lagrange multiplier $\mathbf{u}_{(2)}$, one addresses the functional structure of the Lagrangian $L$ because to single out $\mathbf{u}_{(2)}$ from consistency check of the constraint $\boldsymbol{\Phi}^{(2)}$ requires some non-degeneracy conditions on $L$.

From the Euler-Lagrange equations (37), Lagrange multiplier $\boldsymbol{\lambda}_{1}$ can be solved in terms of the Lagrangian function $L$. Substituting this definition of $\boldsymbol{\lambda}_{1}$ into $L_{C_{1}}$, we obtain an unconstraint Lagrangian function

$$
L_{U_{1}}=L\left(\mathbf{q}_{(1)}, \mathbf{q}_{(2)}, \dot{\mathbf{q}}_{(2)}\right)+\left(\frac{\partial L}{\partial \mathbf{q}_{(2)}}-\frac{d}{d t} \frac{\partial L}{\partial \dot{\mathbf{q}}_{(2)}}\right) \cdot\left(\dot{\mathbf{q}}_{(1)}-\mathbf{q}_{(2)}\right) .
$$

In general, $L_{U_{1}}$ is of the second order, since it involves the total time derivative of $\partial L / \partial \dot{\mathbf{q}}(2)$ which may result with an appearance of the acceleration terms. For a non-degenerate Lagrangian function $L, L_{U_{1}}$ is certainly a second order Lagrangian involving the term $\ddot{\mathbf{q}}_{(2)}$. On the other hand, if Lagrangian function is totally degenerate, that is, if $\partial L / \partial \dot{\mathbf{q}}_{(2)}$ does not depend on $\dot{\mathbf{q}}_{(2)}$, then the term $\partial L / \partial \dot{\mathbf{q}}_{(2)}$ may only depend on $\left(\mathbf{q}_{(1)}, \mathbf{q}_{(2)}\right)$. In this case, the total time derivative will only give the first order terms and $L_{U_{1}}$ turns out to be in the first order form. There still remains some other options on the degree of the unconstraint Lagrangian function from these two extreme cases. All these are related with the degeneracy level of $L_{U_{1}}$. In the forthcoming sections, we shall explicitly present some of these cases on concrete examples. It is needless to say that the dynamics generated by the unconstraint Lagrangian $L_{U_{1}}$ is equivalent to the second order Euler-Lagrange equations (18) independent of its degree. 


\subsubsection{Partial reduction II}

It is interesting to note a tricky point in the definition of $L_{C_{1}}$. In the previous subsection, we have preferred to substitute the velocity component $\dot{\mathbf{q}}$ in $L=L(\mathbf{q} ; \dot{\mathbf{q}} ; \ddot{\mathbf{q}})$ by $\mathbf{q}_{(2)}$ in $(36)$. An alternative way is to take $\dot{\mathbf{q}}$ as $\dot{\mathbf{q}}_{(1)}$. This leads us to another Lagrangian function

$$
L_{C_{2}}=L\left(\mathbf{q}_{(1)}, \dot{\mathbf{q}}_{(1)}, \dot{\mathbf{q}}_{(2)}\right)+\boldsymbol{\lambda}_{2} \cdot\left(\dot{\mathbf{q}}_{(1)}-\mathbf{q}_{(2)}\right),
$$

where $\boldsymbol{\lambda}_{2}$ being a new set of Lagrange multiplier. The Euler-Lagrange equations generated by $L_{C_{2}}$ are computed to be

$$
\frac{\partial L}{\partial \mathbf{q}_{(1)}}-\frac{d}{d t} \frac{\partial L}{\partial \dot{\mathbf{q}}_{(1)}}-\dot{\boldsymbol{\lambda}}_{2}=\mathbf{0}, \quad-\frac{d}{d t} \frac{\partial L}{\partial \dot{\mathbf{q}}_{(2)}}=\boldsymbol{\lambda}_{2}, \quad \dot{\mathbf{q}}_{(1)}-\mathbf{q}_{(2)}=\mathbf{0}
$$

It is immediate to observe that this system is equivalent to the second order Euler-Lagrange equations (18). Even though the Euler-Lagrange equations generated by both $L_{C_{1}}$ and $L_{C_{2}}$ will eventually be the same, the functional structures of the Lagrange multipliers and the unconstraint formalisms will not be the same. The unconstraint Lagrangian for this case is

$$
L_{U_{2}}=L\left(\mathbf{q}_{(1)}, \dot{\mathbf{q}}_{(1)}, \dot{\mathbf{q}}_{(2)}\right)-\frac{d}{d t} \frac{\partial L}{\partial \dot{\mathbf{q}}_{(2)}} \cdot\left(\dot{\mathbf{q}}_{(1)}-\mathbf{q}_{(2)}\right) .
$$

Two different reductions, namely $L_{C_{1}}$ and $L_{C_{2}}$, of the second order Lagrangian $L$ is a manifestation of the double vector bundle structure (6) of the iterated tangent bundle $T T Q$ over the base manifold $T Q$. The first constraint function $L_{C_{1}}$ is the result of the fibration $\tau_{T Q}$ whereas the second one is the result of the fibration $T \tau_{Q}$. Locally, if we choose a local coordinate chart $\left(\mathbf{q}_{(1)}, \mathbf{q}_{(2)} ; \dot{\mathbf{q}}_{(1)}, \dot{\mathbf{q}}_{(2)}\right)$ on $T T Q$, the projections are defined by the two-tuples $\left(\mathbf{q}_{(1)}, \mathbf{q}_{(2)}\right)$ and $\left(\mathbf{q}_{(1)}, \dot{\mathbf{q}}_{(1)}\right)$, respectively. As a result, we may say that recasting a second order Lagrangian as a first order function geometrically corresponds to deciding the base manifold of the iterated bundle $T T Q$. In the following subsection, we shall present another way to define a submanifold of $T T Q$ and the related first order Lagrangian dynamics.

\subsection{Acceleration as a new coordinate}

The main understanding of the previous section is to call the velocity as a new variable. This is not the only way to reduce a second order Lagrangian function to a first order one. In this section, we shall call the acceleration as a new variable.

\subsubsection{Schmidt's method}

Let us start this by the following construction, called as Schmidt's method [67, 68, We refer [2, 3, 29, for discussions on the relationship between the methods of Ostrogradski and Schmidt in pure geometrical terms. Recall acceleration

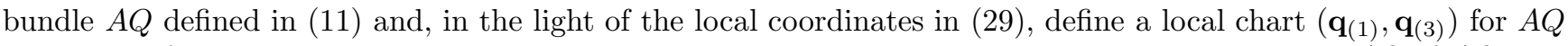
consisting of position $\mathbf{q}_{(1)}$ and acceleration $\mathbf{q}_{(3)}$. The induced coordinates on the tangent bundle $T A Q$ of $A Q$ are

$$
\left(\mathbf{q}_{(1)}, \mathbf{q}_{(3)}, \dot{\mathbf{q}}_{(1)}, \dot{\mathbf{q}}_{(3)}\right) \in T A Q .
$$

Start with a second order Lagrangian function $L$. In order to link the acceleration $\mathbf{q}_{(3)}$ with the derivative of the velocity $\dot{\mathbf{q}}_{(1)}$, introduce a trivial bundle structure $T(A Q \times R)$ over the tangent bundle $T A Q$. Here, $R$ is an $n$-dimensional manifold with local coordinates $(\mathbf{r})$. Define a first order Lagrangian function

$$
L_{3}\left(\mathbf{q}_{(1)}, \mathbf{q}_{(3)}, \dot{\mathbf{q}}_{(1)}, \dot{\mathbf{q}}_{(3)}, \mathbf{r}, \dot{\mathbf{r}}\right)=L\left(\mathbf{q}_{(1)}, \dot{\mathbf{q}}_{(1)}, \mathbf{q}_{(3)}\right)+\frac{\partial F}{\partial \mathbf{q}_{(1)}} \cdot \dot{\mathbf{q}}_{(1)}+\frac{\partial F}{\partial \dot{\mathbf{q}}_{(1)}} \cdot \mathbf{q}_{(3)}+\frac{\partial F}{\partial \mathbf{q}_{(3)}} \cdot \dot{\mathbf{q}}_{(3)}+\frac{\partial F}{\partial \mathbf{r}} \cdot \dot{\mathbf{r}}
$$

on the total space $T(A Q \times R)$. Here, $F$ is an arbitrary function depending on $\left(\mathbf{q}_{(1)}, \mathbf{q}_{(3)}, \dot{\mathbf{q}}_{(1)}, \mathbf{r}\right)$. A direct calculation proves that the first order Euler-Lagrange equations generated by $L_{3}$ on $T(A Q \times M)$ is equivalent to the second order Euler-Lagrange equations (19) only if the matrix $\left[\partial^{2} F / \partial \dot{\mathbf{q}}_{(\mathbf{1})} \partial \mathbf{r}\right]$ is non-degenerate. In order to satisfy this condition, one may simply choose the auxiliary function as $F=\dot{\mathbf{q}}_{(1)} \cdot \mathbf{r}$. Let us proceed with this choice. In this particular case, the Lagrangian function $L_{3}$ in (46) becomes

$$
L_{3}\left(\mathbf{q}_{(1)}, \mathbf{q}_{(3)}, \dot{\mathbf{q}}_{(1)}, \dot{\mathbf{q}}_{(3)}, \mathbf{r}, \dot{\mathbf{r}}\right)=L\left(\mathbf{q}_{(1)}, \dot{\mathbf{q}}_{(1)}, \mathbf{q}_{(3)}\right)+\mathbf{r} \cdot \mathbf{q}_{(3)}+\dot{\mathbf{q}}_{(1)} \cdot \dot{\mathbf{r}}
$$


It is immediate to see that the first order Euler-Lagrange equations generated by $L_{3}$ in (47) is equivalent to the second order Euler-Lagrange equations generated by $L$.

Let us define the conjugate momentum coordinates on the cotangent bundle $T^{*}(A Q \times R)$ by three-tuple $\left(\mathbf{p}_{(1)}, \mathbf{p}_{(2)}, \mathbf{p}_{(r)}\right)$ which can be computed as

$$
\mathbf{p}^{(1)}=\frac{\partial L}{\partial \dot{\mathbf{q}}_{(1)}}\left(\mathbf{q}_{(1)}, \dot{\mathbf{q}}_{(1)}, \mathbf{q}_{(3)}\right)+\dot{\mathbf{r}}, \quad \mathbf{p}^{(3)}=\mathbf{0}, \quad \mathbf{p}^{(r)}=\dot{\mathbf{q}}^{(1)} .
$$

It is evident that, we can solve $\dot{\mathbf{r}}$ in terms of $\left(\dot{\mathbf{q}}_{(1)}, \dot{\mathbf{q}}_{(3)}, \mathbf{p}^{(1)}, \mathbf{p}^{(r)}\right)$. See also that $\varphi_{1}=\mathbf{p}^{(3)} \approx 0$ is a primary constraint. Accordingly, we define the following total Hamiltonian function

$$
H_{T}=\mathbf{p}^{(1)} \cdot \mathbf{p}^{(r)}-L\left(\mathbf{q}_{(1)}, \mathbf{p}^{(r)}, \mathbf{q}_{(3)}\right)-\mathbf{r} \cdot \mathbf{q}_{(3)}+\mathbf{u} \cdot \mathbf{p}^{(3)},
$$

where $\mathbf{u}$ being Lagrange multipliers. Check the consistency of the primary constraint as

$$
\varphi_{2}=\left\{\mathbf{p}^{(3)}, H_{T}\right\}=\frac{\partial L}{\partial \mathbf{q}_{(3)}}+\mathbf{r}=\mathbf{0} .
$$

Consistency of the secondary constraint $\varphi_{2}$ results with a tertiary constraint

$$
\varphi_{3}=\left\{\varphi_{2}, H_{T}\right\}=\left(\mathbf{u} \cdot \frac{\partial}{\partial \mathbf{q}_{(3)}}+\mathbf{q}_{(3)} \cdot \frac{\partial}{\partial \mathbf{p}^{(r)}}+\mathbf{p}^{(r)} \cdot \frac{\partial}{\partial \mathbf{q}_{(1)}}\right)\left(\frac{\partial L}{\partial \mathbf{q}_{(3)}}\right)+\mathbf{p}^{(r)}=0 .
$$

If the Lagrangian is non-degenerate in the sense of Ostrogradski then this step determines the Lagrange multipliers $\mathbf{u}$, and the constraint algorithm is finished up. If the Lagrangian is degenerate further steps may be needed to determine the Lagrange multipliers as well as to close up the Poisson algebra.

\subsubsection{Deriglazov's trick}

Now, we apply a trick due to Deriglazov to reduce the second order Lagrangian function $L=L(\mathbf{q}, \dot{\mathbf{q}}, \ddot{\mathbf{q}})$ to a first order one by following [23. We introduce the action integral

$$
\int\left(L\left(\mathbf{q}_{(1)}, \dot{\mathbf{q}}_{(1)}, \mathbf{q}_{(3)}\right)+\gamma \cdot\left(\ddot{\mathbf{q}}_{(1)}-\mathbf{q}_{(3)}\right)\right) d t,
$$

where $\gamma$ is a set of Lagrange multipliers. Applying the by-parts technique to the second term inside the integral, we arrive at the following reduced Lagrangian function

$$
L_{4}=L\left(\mathbf{q}_{(1)}, \dot{\mathbf{q}}_{(1)}, \mathbf{q}_{(3)}\right)-\dot{\gamma} \cdot \dot{\mathbf{q}}_{(1)}-\gamma \cdot \mathbf{q}_{(3)}
$$

on the extended velocity phase space $T(A Q \times R)$ with coordinates $\left(\mathbf{q}_{(1)}, \mathbf{q}_{(3)}, \boldsymbol{\gamma} ; \dot{\mathbf{q}}_{(1)}, \dot{\mathbf{q}}_{(3)}, \dot{\gamma}\right)$. It is immediate to observe that the first order Euler-Lagrange equations generated by $L_{4}$ is equivalent to the second order EulerLagrange equations (18) generated by $L$.

The Schmidt's method and Deriglazov's trick are the same in the particular cases we are interested in, that is degenerate second order Lagrangian functions. To see this, compare the Lagrangian functions $L_{3}$ and $L_{4}$ presented in (47) and (53). These two Lagrangian functions are the same if a simple identification $\gamma=-\mathbf{r}$ is performed. This results with the identification $\dot{\gamma}=-\dot{\mathbf{r}}$ as well.

\section{Clèment Lagrangian}

Let us recall here degenerate second order Clèment's Lagrangian function

$$
L^{C}[\mathbf{x}]=-\frac{m}{2} \zeta\|\dot{\mathbf{x}}\|^{2}-\frac{2 m \Lambda}{\zeta}+\frac{\zeta^{2}}{2 \mu m} \mathbf{x} \cdot(\dot{\mathbf{x}} \times \ddot{\mathbf{x}})
$$

on the second order tangent bundle $T^{2} Q$ with local coordinates $[\mathbf{x}]=(\mathbf{x}, \dot{\mathbf{x}}, \ddot{\mathbf{x}})$. Here, the inner product $\|\mathbf{x}\|^{2}=$ $T^{2}-x^{2}-y^{2}$ is defined by the Lorentzian metric and the triple product is $\mathbf{x} \cdot(\dot{\mathbf{x}} \times \ddot{\mathbf{x}})=\epsilon_{i j k} x^{i} \dot{x}^{j} \ddot{x}^{k}$ where $\epsilon_{i j k}$ is 
the completely antisymmetric tensor of rank three. Dot denotes the derivative with respect to the variable $t$ and $\zeta=\zeta(t)$ is a function which allows arbitrary reparametrization of the variable $t . \Lambda$ and $1 / 2 m$ are cosmological and Einstein gravitational constants, respectively.

The variation of Clèment Lagrangian (54) with respect to $\zeta$ gives the energy constraint

$$
E^{C}[\mathbf{x}]=-\frac{m}{2}\|\dot{\mathbf{x}}\|^{2}+2 \frac{m \Lambda}{\zeta^{2}}+\frac{\zeta}{m \mu} \mathbf{x} \cdot(\dot{\mathbf{x}} \times \ddot{\mathbf{x}})=0 .
$$

whereas the variation of the Lagrangian (54) with respect to $\mathbf{x}$ results with the Euler-Lagrange equations

$$
2 m^{2} \mu \ddot{\mathbf{x}}+3 \dot{\mathbf{x}} \times \ddot{\mathbf{x}}+2 \mathbf{x} \times \mathbf{x}^{(3)}=\mathbf{0}
$$

which is a third order differential equation. In the Euler-Lagrange equations (56), we set the reparametrization function $\zeta$ equal to one. The Clèment Lagrangian (54) is invariant under translations in $t$ and pseudo-rotations in space. Time translation symmetry gives the conservation of energy.

In the following subsections, we shall apply four reductions methods, namely, the total reduction, partial reduction I, partial reduction II, and Deriglazov's trick / Schmidt's method, presented in the previous section to the case of the Clèment Lagrangian (54). Then we shall obtain their Hamiltonian realizations by employing the Dirac-Bergmann constraint algorithm. For each of the methods, we shall present the Dirac brackets. In addition, we shall exhibit the unconstraint Lagrangian realizations for the case of partial reductions and write the associated Hamiltonian formalisms.

\subsection{Total Reduction}

Recall that, in (3.1), we have presented the total reduction of a second order Lagragian function. For the case of the Clèment Lagrangian in (54), this reads the following first order Lagrangian function

$$
L_{C}=-\frac{m \zeta}{2}\left\|\mathbf{q}_{(2)}\right\|^{2}+\frac{\zeta^{2}}{2 \mu m} \mathbf{q}_{(1)} \cdot \mathbf{q}_{(2)} \times \mathbf{q}_{(3)}+\boldsymbol{\lambda}_{1} \cdot\left(\dot{\mathbf{q}}_{(1)}-\mathbf{q}_{(2)}\right)+\boldsymbol{\lambda}_{2} \cdot\left(\dot{\mathbf{q}}_{(2)}-\mathbf{q}_{(3)}\right)
$$

with coordinates $\mathbf{q}_{(1)}=\mathbf{x}, \mathbf{q}_{(2)}=\dot{\mathbf{x}}$, and $\mathbf{q}_{(3)}=\ddot{\mathbf{x}}$. Here, $\boldsymbol{\lambda}_{1}$ and $\boldsymbol{\lambda}_{2}$ are Lagrange multipliers. Variation of $L_{C}$ with respect to $\mathbf{q}_{(1)}, \mathbf{q}_{(2)}$ and $\mathbf{q}_{(3)}$ results in the Euler-Lagrange equations

$$
\frac{\zeta^{2}}{2 \mu m} \mathbf{q}_{(2)} \times \mathbf{q}_{(3)}-\dot{\boldsymbol{\lambda}}_{1}=\mathbf{0}, \quad-m \zeta \mathbf{q}_{(2)}+\frac{\zeta^{2}}{2 \mu m} \mathbf{q}_{(3)} \times \mathbf{q}_{(1)}-\boldsymbol{\lambda}_{1}-\dot{\boldsymbol{\lambda}}_{2}=\mathbf{0}, \quad \frac{\zeta^{2}}{2 \mu m} \mathbf{q}_{(1)} \times \mathbf{q}_{(2)}-\boldsymbol{\lambda}_{2}=\mathbf{0} .
$$

We introduce the conjugate momenta $\left(\mathbf{p}^{(1)}, \mathbf{p}^{(2)}, \mathbf{p}^{(3)}, \mathbf{p}^{\left(\lambda_{1}\right)}, \mathbf{p}^{\left(\lambda_{2}\right)}\right)$. The Legendre transformation results with the following identities

$$
\mathbf{p}^{(1)}=\frac{\partial L_{C}}{\partial \dot{\mathbf{q}}_{(1)}}=\boldsymbol{\lambda}_{1}, \quad \mathbf{p}^{(2)}=\frac{\partial L_{C}}{\partial \dot{\mathbf{q}}_{(2)}}=\boldsymbol{\lambda}_{2}, \quad \mathbf{p}^{(3)}=\frac{\partial L_{C}}{\partial \dot{\mathbf{q}}_{(3)}}=0, \quad \mathbf{p}^{\left(\lambda_{1}\right)}=\frac{\partial L_{C}}{\partial \dot{\boldsymbol{\lambda}}_{(1)}}=0, \quad \mathbf{p}^{\left(\lambda_{2}\right)}=\frac{\partial L_{C}}{\partial \dot{\boldsymbol{\lambda}}_{(2)}}=\mathbf{0} .
$$

Definition of momenta in (59) imply the set of primary constraints

$$
\boldsymbol{\Phi}^{(1)}=\mathbf{p}^{(1)}-\boldsymbol{\lambda}_{1}, \quad \boldsymbol{\Phi}^{(2)}=\mathbf{p}^{(2)}-\boldsymbol{\lambda}_{2}, \quad \boldsymbol{\Phi}^{(3)}=\mathbf{p}^{(3)}, \quad \boldsymbol{\Phi}^{\left(\lambda_{1}\right)}=\mathbf{p}^{\left(\lambda_{1}\right)}, \quad \boldsymbol{\Phi}^{\left(\lambda_{2}\right)}=\mathbf{p}^{\left(\lambda_{2}\right)} .
$$

Let us now introduce the canonical Hamiltonian function

$$
\begin{aligned}
H & =\mathbf{p}^{(1)} \cdot \dot{\mathbf{q}}_{(1)}+\mathbf{p}^{(2)} \cdot \dot{\mathbf{q}}_{(2)}+\mathbf{p}^{(3)} \cdot \dot{\mathbf{q}}_{(3)}+\mathbf{p}^{\left(\lambda_{1}\right)} \cdot \dot{\boldsymbol{\lambda}}_{(1)}+\mathbf{p}^{\left(\lambda_{2}\right)} \cdot \dot{\boldsymbol{\lambda}}_{(2)}-L_{C} \\
& =\mathbf{p}^{(1)} \cdot \dot{\mathbf{q}}_{(1)}+\mathbf{p}^{(2)} \cdot \dot{\mathbf{q}}_{(2)}+\mathbf{p}^{(3)} \cdot \dot{\mathbf{q}}_{(3)}+\mathbf{p}^{\left(\lambda_{1}\right)} \cdot \dot{\boldsymbol{\lambda}}_{(1)}+\mathbf{p}^{\left(\lambda_{2}\right)} \cdot \dot{\boldsymbol{\lambda}}_{(2)}+\frac{m \zeta}{2}\left\|\mathbf{q}_{(2)}\right\|^{2}-\frac{\zeta^{2}}{2 \mu m} \mathbf{q}_{(1)} \cdot \mathbf{q}_{(2)} \times \mathbf{q}_{(3)} \\
& -\boldsymbol{\lambda}_{1} \cdot\left(\dot{\mathbf{q}}_{(1)}-\mathbf{q}_{(2)}\right)-\boldsymbol{\lambda}_{2} \cdot\left(\dot{\mathbf{q}}_{(2)}-\mathbf{q}_{(3)}\right) .
\end{aligned}
$$

After some algebraic manipulations we arrive at

$$
\begin{aligned}
H & =\left(\mathbf{p}^{(1)}-\boldsymbol{\lambda}_{1}\right) \cdot \dot{\mathbf{q}}_{(1)}+\left(\mathbf{p}^{(2)}-\boldsymbol{\lambda}_{2}\right) \cdot \dot{\mathbf{q}}_{(2)}+\mathbf{p}^{(3)} \cdot \dot{\mathbf{q}}_{(3)}+\mathbf{p}^{\left(\lambda_{1}\right)} \cdot \dot{\boldsymbol{\lambda}}_{(1)}+\mathbf{p}^{\left(\lambda_{2}\right)} \cdot \dot{\boldsymbol{\lambda}}_{(2)} \\
& +\frac{m \zeta}{2}\left\|\mathbf{q}_{(2)}\right\|^{2}-\frac{\zeta^{2}}{2 \mu m} \mathbf{q}_{(1)} \cdot \mathbf{q}_{(2)} \times \mathbf{q}_{(3)}+\boldsymbol{\lambda}_{1} \cdot \mathbf{q}_{(2)}+\boldsymbol{\lambda}_{2} \cdot \mathbf{q}_{(3)} .
\end{aligned}
$$


By substituting the primary constraints, we define the total Hamiltonian function as

$$
H_{T}=H+\boldsymbol{\Phi}^{(1)} \cdot \mathbf{U}_{1}+\boldsymbol{\Phi}^{(2)} \cdot \mathbf{U}_{2}+\boldsymbol{\Phi}^{(3)} \cdot \mathbf{U}_{3}+\boldsymbol{\Phi}^{\left(\lambda_{1}\right)} \cdot \mathbf{U}_{\lambda_{1}}+\boldsymbol{\Phi}^{\left(\lambda_{2}\right)} \cdot \mathbf{U}_{\lambda_{2}}
$$

where

$$
H=\frac{m \zeta}{2}\left\|\mathbf{q}_{(2)}\right\|^{2}-\frac{\zeta^{2}}{2 \mu m} \mathbf{q}_{(1)} \cdot \mathbf{q}_{(\mathbf{2})} \times \mathbf{q}_{(3)}+\boldsymbol{\lambda}_{1} \cdot \mathbf{q}_{(2)}+\boldsymbol{\lambda}_{2} \cdot \mathbf{q}_{(3)}
$$

is the canonical Hamiltonian function, and $\mathbf{U}_{1}, \mathbf{U}_{2}, \mathbf{U}_{3}, \mathbf{U}_{\lambda_{1}}, \mathbf{U}_{\lambda_{2}}$ are the Lagrange multipliers. Here are the steps of the Dirac-Bergmann Constraint algorithm and the computation of the total Hamiltonian function.

Dirac-Bergmann constraint algorithm step 1: Consistency checks of the primary constraints $\boldsymbol{\Phi}^{(1)}, \boldsymbol{\Phi}^{(2)}, \boldsymbol{\Phi}^{\left(\lambda_{1}\right)}$ and $\boldsymbol{\Phi}^{\left(\lambda_{2}\right)}$ result with

$$
\begin{aligned}
\dot{\boldsymbol{\Phi}}^{(1)} & =\left\{\boldsymbol{\Phi}^{(1)}, H_{T}\right\} \approx \frac{\zeta^{2}}{2 \mu m} \mathbf{q}_{(2)} \times \mathbf{q}_{(3)}-\mathbf{U}_{\lambda_{1}} \\
\dot{\boldsymbol{\Phi}}^{(2)} & =\left\{\boldsymbol{\Phi}^{(2)}, H_{T}\right\} \approx-m \zeta \mathbf{q}_{(2)}+\frac{\zeta^{2}}{2 \mu m} \mathbf{q}_{(3)} \times \mathbf{q}_{(1)}-\lambda_{1}-\mathbf{U}_{\lambda_{2}} \\
\dot{\boldsymbol{\Phi}}^{\left(\lambda_{1}\right)} & =\left\{\boldsymbol{\Phi}^{\left(\lambda_{1}\right)}, H_{T}\right\} \approx \mathbf{U}_{1}-\mathbf{q}_{(2)}, \\
\dot{\boldsymbol{\Phi}}^{\left(\lambda_{2}\right)} & =\left\{\boldsymbol{\Phi}^{\left(\lambda_{2}\right)}, H_{T}\right\} \approx \mathbf{U}_{2}-\mathbf{q}_{(3)}
\end{aligned}
$$

which lead to determine $\mathbf{U}_{1}, \mathbf{U}_{2}, \mathbf{U}_{\lambda_{1}}, \mathbf{U}_{\lambda_{2}}$. Consistency checks of the primary constraints $\boldsymbol{\Phi}^{(3)}$ determine a set of secondary constraints

$$
\boldsymbol{\Phi}=\dot{\boldsymbol{\Phi}}^{(3)}=\left\{\boldsymbol{\Phi}^{(3)}, H_{T}\right\} \approx \frac{\zeta^{2}}{2 \mu m} \mathbf{q}_{(1)} \times \mathbf{q}_{(2)}-\boldsymbol{\lambda}_{2} .
$$

Accordingly, we revise the total Hamiltonian $H_{T}$ by adding the secondary constraint $\mathbf{\Phi}$ with a set $\mathbf{U}$ of Lagrange multipliers. This reads $H_{T}^{1}=H_{T}+\mathbf{U} \cdot \boldsymbol{\Phi}$.

Dirac-Bergmann constraint algorithm step 2: Consistency checks of the secondary constraints $\boldsymbol{\Phi}$ determine another set of constraints

$$
\boldsymbol{\varphi}=\dot{\boldsymbol{\Phi}}=\left\{\boldsymbol{\Phi}, H_{T}^{1}\right\} \approx m \zeta \mathbf{q}_{(2)}+\frac{\zeta^{2}}{\mu m} \mathbf{q}_{(1)} \times \mathbf{q}_{(3)}+\boldsymbol{\lambda}_{1}
$$

We revise the total Hamiltonian function as $H_{T}^{2}=H_{T}^{1}+\mathbf{V} \cdot \boldsymbol{\varphi}$ where $\mathbf{V}$ being a set of Lagrange multipliers.

Dirac-Bergmann constraint algorithm step 3: The conservation of $\varphi$ gives

$$
\dot{\boldsymbol{\varphi}}=\left\{\boldsymbol{\varphi}, H_{T}^{2}\right\} \approx m \zeta \mathbf{q}_{(3)}+\frac{3 \zeta^{2}}{2 \mu m} \mathbf{q}_{(2)} \times \mathbf{q}_{(3)}+\frac{\zeta^{2}}{\mu m} \mathbf{q}_{(1)} \times \mathbf{U}_{(3)} .
$$

From this, we can determine two components of $\mathbf{U}_{(3)}$ while we arrive at a scalar constraint by simply taking the dot product of $\dot{\varphi}$ with $\mathbf{q}_{(1)}$, that is

$$
\chi=\mathbf{q}_{(1)} \cdot\left(m \zeta \mathbf{q}_{(3)}+\frac{3 \zeta^{2}}{2 \mu m} \mathbf{q}_{(2)} \times \mathbf{q}_{(3)}\right) .
$$

We arrive at the total Hamiltonian function $H_{T}^{3}=H_{T}^{2}+W \chi$. Here, $W$ is a Lagrange multiplier.

Dirac-Bergmann constraint algorithm step 4: At final, the conservation of $\chi$ gives the third component of $\mathbf{U}_{(3)}$ and the remaining constraints determine the other Lagrange multipliers. A direct calculation determines the Lagrange multiplier $\mathbf{U}, \mathbf{V}, \mathbf{W}$ and $\mathbf{U}_{(3)}$ as

$$
\begin{aligned}
\mathbf{U} & \approx \mathbf{0}, \quad \mathbf{V} \approx \mathbf{0}, \quad \mathbf{W} \approx \mathbf{0} \\
\mathbf{U}_{(3)} & \approx \mathbf{q}_{(1)}\left(\frac{\mathbf{q}_{(2)} \cdot \mathbf{q}_{(3)}}{\left\|\mathbf{q}_{(1)}\right\|^{2}}\right)-\frac{\mu}{\zeta^{3}\left\|\mathbf{q}_{(1)}\right\|^{2}}\left[\frac{3 \zeta^{2}}{2 \mu m} \mathbf{q}_{(2)} \times \mathbf{q}_{(3)}+m \zeta \mathbf{q}_{(3)}\right] \times\left[\frac{3 \zeta^{2}}{2 \mu m} \mathbf{q}_{(1)} \times \mathbf{q}_{(2)}+m \zeta \mathbf{q}_{(1)}\right]
\end{aligned}
$$

under the assumption $\left\|\mathbf{q}_{(1)}\right\|^{2} \neq 0$. The following table summarizes the discussions have been done so far. 


\begin{tabular}{|c|c|}
\hline & Total Reduction \\
\hline Reduction & $\mathbf{x}=\mathbf{q}_{(1)}, \dot{\mathbf{x}}=\mathbf{q}_{(2)}, \ddot{\mathbf{x}}=\mathbf{q}_{(3)}$, \\
\hline Coordinates & $\mathbf{q}_{(1)}, \mathbf{q}_{(2)}, \mathbf{q}_{(3)}, \boldsymbol{\lambda}_{1}, \boldsymbol{\lambda}_{2}, \mathbf{p}^{(1)}, \mathbf{p}^{(2)}, \mathbf{p}^{(3)}, \mathbf{p}^{\left(\lambda_{1}\right)}, \mathbf{p}^{\left(\lambda_{2}\right)}$ \\
\hline \multirow{4}{*}{ Primary Constraints } & $\boldsymbol{\Phi}^{(1)}=\mathbf{p}^{(1)}-\boldsymbol{\lambda}_{1} \mathbf{\Phi}^{\left(\lambda_{1}\right)}=\mathbf{p}^{\left(\lambda_{1}\right)}$ \\
& $\mathbf{\Phi}^{(2)}=\mathbf{p}^{(2)}-\boldsymbol{\lambda}_{2} \quad \mathbf{\Phi}^{\left(\lambda_{2}\right)}=\mathbf{p}^{\left(\lambda_{2}\right)}$ \\
& $\boldsymbol{\Phi}^{(3)}=\mathbf{p}^{(3)}$ \\
\hline Secondary Constraints & $\mathbf{\Phi}=\frac{\zeta^{2}}{2 \mu m} \mathbf{q}_{(1)} \times \mathbf{q}_{(2)}-\boldsymbol{\lambda}_{2}$ \\
\hline Tertiary Constraints & $\boldsymbol{\varphi}=m \zeta \mathbf{q}_{(2)}+\frac{\zeta^{2}}{\mu m} \mathbf{q}_{(1)} \times \mathbf{q}_{(3)}+\boldsymbol{\lambda}_{1}$ \\
\hline Quaternary Constraint & $\chi=\mathbf{q}_{(1)} \cdot\left(m \zeta \mathbf{q}_{(3)}+\frac{3 \zeta^{2}}{2 \mu m} \mathbf{q}_{(2)} \times \mathbf{q}_{(3)}\right)$ \\
\hline
\end{tabular}

The total Hamiltonian function and the equations of motion: Let us substitute the Lagrange multipliers $\mathbf{U}_{1}, \mathbf{U}_{2}, \mathbf{U}_{3}, \mathbf{U}_{\lambda_{1}}, \mathbf{U}_{\lambda_{2}}, \mathbf{U}, \mathbf{V}, \mathbf{W}$ determined in the constraint algorithm into the total Hamiltonian function $H_{T}^{3}$. So that we have the following explicit expression

$$
\begin{aligned}
H_{T}^{3} & =\frac{m \zeta}{2}\left\|\mathbf{q}_{(2)}\right\|^{2}-\frac{\zeta^{2}}{2 \mu m} \mathbf{q}_{(1)} \cdot \mathbf{q}_{(2)} \times \mathbf{q}_{(3)}+\mathbf{q}_{(2)} \cdot \mathbf{p}^{(1)}+\mathbf{q}_{(3)} \cdot \mathbf{p}^{(2)} \\
& +\left[\mathbf{q}_{(1)}\left(\frac{\mathbf{q}_{(2)} \cdot \mathbf{q}_{(3)}}{\left\|\mathbf{q}_{(1)}\right\|^{2}}\right)-\frac{\mu}{\zeta^{3}\left\|\mathbf{q}_{(1)}\right\|^{2}}\left[\frac{3 \zeta^{2}}{2 \mu m} \mathbf{q}_{(2)} \times \mathbf{q}_{(3)}+m \zeta \mathbf{q}_{(3)}\right] \times\left[\frac{3 \zeta^{2}}{2 \mu m} \mathbf{q}_{(1)} \times \mathbf{q}_{(2)}+m \zeta \mathbf{q}_{(1)}\right]\right] \cdot \mathbf{p}^{(3)} \\
& +\frac{\zeta^{2}}{2 \mu m} \mathbf{p}^{\left(\lambda_{1}\right)} \cdot \mathbf{q}_{(2)} \times \mathbf{q}_{(3)}+\left[-m \zeta \mathbf{q}_{(2)}+\frac{\zeta^{2}}{2 \mu m} \mathbf{q}_{(3)} \times \mathbf{q}_{(1)}-\boldsymbol{\lambda}_{1}\right] \cdot \mathbf{p}^{\left(\lambda_{2}\right)} .
\end{aligned}
$$

So that the Hamilton's equations of motion governed by the total Hamiltonian in (71) are computed to be

$$
\begin{aligned}
\dot{\mathbf{q}}_{(1)} & \approx \mathbf{q}_{(2)}, \quad \dot{\mathbf{q}}_{(2)} \approx \mathbf{q}_{(3)}, \\
\dot{\mathbf{q}}_{(3)} & \approx \mathbf{q}_{(1)}\left(\frac{\mathbf{q}_{(2)} \cdot \mathbf{q}_{(3)}}{\left\|\mathbf{q}_{(1)}\right\|^{2}}\right)-\frac{\mu}{\zeta^{3}\left\|\mathbf{q}_{(1)}\right\|^{2}}\left[\frac{3 \zeta^{2}}{2 \mu m} \mathbf{q}_{(2)} \times \mathbf{q}_{(3)}+m \zeta \mathbf{q}_{(3)}\right] \times\left[\frac{3 \zeta^{2}}{2 \mu m} \mathbf{q}_{(1)} \times \mathbf{q}_{(2)}+m \zeta \mathbf{q}_{(1)}\right], \\
\dot{\mathbf{p}}^{(1)} & =\dot{\boldsymbol{\lambda}}_{1} \approx \frac{\zeta^{2}}{2 \mu m} \mathbf{q}_{(2)} \times \mathbf{q}_{(3)}, \\
\dot{\mathbf{p}}^{(2)} & =\dot{\boldsymbol{\lambda}}_{2} \approx-m \zeta \mathbf{q}_{(2)}+\frac{\zeta^{2}}{2 \mu m} \mathbf{q}_{(3)} \times \mathbf{q}_{(1)}-\lambda_{1} \quad \dot{\mathbf{p}}^{(3)} \approx \frac{\zeta^{2}}{2 \mu m} \mathbf{q}_{(1)} \times \mathbf{q}_{(2)}-\boldsymbol{\lambda}_{2}, \\
\dot{\mathbf{p}}^{\left(\lambda_{1}\right)} & \approx 0, \quad \dot{\mathbf{p}}^{\left(\lambda_{2}\right)} \approx 0 .
\end{aligned}
$$

In order to retrieve the Euler-Lagrange equations (56) generated by the Clèment Lagrangian from the Hamilton's equations, we simply substitute the momenta into the equation of motion governing $\mathbf{p}^{(\mathbf{1})}$. The rest of the equations are then trivially satisfied.

\section{The Dirac bracket:}

We can also derive the Hamilton's equations using Dirac bracket. All constraints for the Total reduction case are

$$
\begin{aligned}
& \boldsymbol{\Phi}^{(1)}=\mathbf{p}^{(1)}-\lambda_{1}, \quad \boldsymbol{\Phi}^{\left(\lambda_{1}\right)}=\mathbf{p}^{\left(\lambda_{1}\right)}, \quad \boldsymbol{\Phi}^{(2)}=\mathbf{p}^{(2)}-\boldsymbol{\lambda}_{2}, \quad \boldsymbol{\Phi}^{\left(\lambda_{2}\right)}=\mathbf{p}^{\left(\lambda_{2}\right)}, \quad \boldsymbol{\Phi}^{(3)}=\mathbf{p}^{(3)}, \\
& \boldsymbol{\Phi}=\frac{\zeta^{2}}{2 \mu m} \mathbf{q}_{(1)} \times \mathbf{q}_{(2)}-\boldsymbol{\lambda}_{2}, \quad \boldsymbol{\varphi}=m \zeta \mathbf{q}_{(2)}+\frac{\zeta^{2}}{\mu m} \mathbf{q}_{(1)} \times \mathbf{q}_{(3)}+\boldsymbol{\lambda}_{1}, \quad \chi=\mathbf{q}_{(1)} \cdot\left(m \zeta \mathbf{q}_{(3)}+\frac{3 \zeta^{2}}{2 \mu m} \mathbf{q}_{(2)} \times \mathbf{q}_{(3)}\right)
\end{aligned}
$$


second class. Recalling the definition in equation (27) of the Dirac bracket, we derive some of Dirac brackets

$$
\begin{aligned}
& \left\{q_{(1)}^{i}, q_{(1)}^{j}\right\}_{D B}=\left\{q_{(1)}^{i}, \lambda_{(2)}^{j}\right\}_{D B}=\left\{q_{(1)}^{i}, p_{j}^{(2)}\right\}_{D B}=\left\{q_{(1)}^{i}, p_{j}^{(3)}\right\}_{D B}=\left\{q_{(1)}^{i}, p_{j}^{\left(\lambda_{1}\right)}\right\}_{D B}=\left\{q_{(1)}^{i}, p_{j}^{\left(\lambda_{2}\right)}\right\}_{D B} \\
& =\left\{q_{(2)}^{i}, p_{j}^{\left(\lambda_{1}\right)}\right\}_{D B}=\left\{q_{(2)}^{i}, p_{j}^{\left(\lambda_{2}\right)}\right\}_{D B}=\left\{q_{(2)}^{i}, p_{j}^{(3)}\right\}_{D B}=\left\{\lambda_{(1)}^{i}, p_{j}^{(3)}\right\}_{D B}=\left\{\lambda_{(2)}^{i}, p_{j}^{(3)}\right\}_{D B} \\
& =\left\{\lambda_{(1)}^{i}, p_{j}^{\left(\lambda_{1}\right)}\right\}_{D B}=\left\{\lambda_{(1)}^{i}, p_{j}^{\left(\lambda_{2}\right)}\right\}_{D B}=\left\{\lambda_{(2)}^{i}, p_{j}^{\left(\lambda_{1}\right)}\right\}_{D B}=\left\{\lambda_{(2)}^{i}, p_{j}^{\left(\lambda_{2}\right)}\right\}_{D B}=0 \\
& \left\{q_{(1)}^{i}, q_{(2)}^{j}\right\}_{D B}=-\frac{1}{m \zeta\left\|\mathbf{q}_{(1)}\right\|^{2}} q_{(1)}^{i} q_{(1)}^{j} \\
& \left\{q_{(1)}^{i}, \lambda_{(1)}^{j}\right\}_{D B}=\left\{q_{(1)}^{i}, \delta^{j k} p_{k}^{(1)}\right\}_{D B}=\delta^{i j}+\frac{\zeta}{2 \mu m^{2}\left\|\mathbf{q}_{(1)}\right\|^{2}} q_{(1)}^{i} \delta^{j k} \epsilon_{k l r} q_{(1)}^{l} q_{(2)}^{r} \\
& \left\{q_{(2)}^{i}, q_{(2)}^{j}\right\}_{D B}=-\frac{\mu m}{\zeta^{2}\left\|\mathbf{q}_{(1)}\right\|^{2}} \epsilon_{i j k} q_{(1)}^{k}-\frac{1}{2 m \zeta\left\|\mathbf{q}_{(1)}\right\|^{2}} \epsilon^{i j k} \epsilon_{k l r} q_{(1)}^{l} q_{(2)}^{r} \\
& \left\{q_{(2)}^{i}, \lambda_{(1)}^{j}\right\}_{D B}=\frac{-q_{(1)}^{j} q_{(2)}^{i}+\delta^{i j} \mathbf{q}_{(\mathbf{1})} \cdot \mathbf{q}_{(\mathbf{2})}}{2\left\|\mathbf{q}_{(1)}\right\|^{2}}+\frac{\zeta}{4 \mu m^{2}\left\|\mathbf{q}_{(1)} \mid\right\|^{2}} q_{(2)}^{i} \delta^{j r} \epsilon_{r k l} q_{(2)}^{k} q_{(1)}^{l}+\frac{\zeta}{\mu m^{2}\left\|\mathbf{q}_{(1)}\right\|^{2}} q_{(1)}^{i} \delta^{j r} \epsilon_{r k l} q_{(1)}^{k} q_{(3)}^{l} \\
& \left\{q_{(2)}^{i}, \lambda_{(2)}^{j}\right\}_{D B}=\left\{q_{(2)}^{i}, \delta^{j k} p_{k}^{(2)}\right\}_{D B}=\frac{-q_{(1)}^{j} q_{(1)}^{i}+\delta^{i j}\left\|\mathbf{q}_{(\mathbf{1})}\right\|^{2}}{2\left\|\mathbf{q}_{(1)}\right\|^{2}}+\frac{\zeta}{4 \mu m^{2}\left\|\mathbf{q}_{(1)}\right\|^{2}} q_{(1)}^{i} \delta^{j r} \epsilon_{r k l} q_{(1)}^{k} q_{(2)}^{l} \\
& \left\{\lambda_{(1)}^{i}, \lambda_{(1)}^{j}\right\}_{D B}=\left\{\lambda_{(1)}^{i}, \delta^{j k} p_{k}^{(1)}\right\}_{D B}=-\frac{\zeta^{2}}{4 \mu m\left\|\mathbf{q}_{(1)}\right\|^{2}} \epsilon^{i j k} \delta_{k l} q_{(1)}^{l}\left(\mathbf{q}_{(\mathbf{1})} \cdot \mathbf{q}_{(\mathbf{2})}\right)+\frac{\zeta^{3}}{2 \mu^{2} m^{3}\left\|\mathbf{q}_{(1)}\right\|^{2}} \epsilon^{i j k} \delta_{k l} q_{(1)}^{l}\left(\mathbf{q}_{(1)} \cdot \mathbf{q}_{(3)} \times \mathbf{q}_{(2)}\right) \\
& \left\{\lambda_{(1)}^{i}, \lambda_{(2)}^{j}\right\}_{D B}=\left\{\lambda_{(1)}^{i}, \delta^{j k} p_{k}^{(2)}\right\}_{D B}=\left\{\delta^{i k} p_{k}^{(1)}, \lambda_{(2)}^{j},\right\}_{D B} \\
& =\frac{\zeta^{2}}{4 \mu m\left\|\mathbf{q}_{(1)}\right\|^{2}} \delta^{i r} \epsilon_{r k l} q_{(1)}^{k} q_{(2)}^{l}+\frac{\zeta^{2}}{4 \mu m} \epsilon^{i j k} \delta_{k l} q_{(2)}^{l}+\frac{\zeta^{3}}{8 \mu^{2} m^{3}\left\|\mathbf{q}_{(1)}\right\|^{2}} \delta^{i r} \epsilon_{r k l} q_{(2)}^{k} q_{(1)}^{l} \delta^{j r^{\prime}} \epsilon_{r^{\prime} k^{\prime} l^{\prime}} q_{(1)}^{k^{\prime}} q_{(2)}^{l^{\prime}} \\
& \left\{\lambda_{(2)}^{i}, \lambda_{(2)}^{j}\right\}_{D B}=\left\{\lambda_{(2)}^{i}, \delta^{j k} p_{k}^{(2)}\right\}_{D B}=-\frac{\zeta^{2}}{4 \mu m} \epsilon^{i j k} \delta_{k l} q_{(1)}^{l}
\end{aligned}
$$

which are required to derive Hamilton equations. Equations of motion generated by canonical Hamiltonian function $\mathrm{H}$ given in (64) can be evaluated from

$$
\begin{aligned}
\dot{X}^{i}=\left\{X^{i}, H\right\}_{D B} & =-\frac{\zeta^{2}}{2 \mu m} \epsilon_{j k l} q_{(2)}^{k} q_{(3)}^{l}\left\{X^{i}, q_{(1)}^{j}\right\}_{D B}+\frac{\zeta^{2}}{2 \mu m} \epsilon_{j k l} q_{(3)}^{k} q_{(1)}^{l}\left\{X^{i}, q_{(2)}^{j}\right\}_{D B} \\
& +\delta_{j k} q_{(2)}^{k}\left\{X^{i}, \lambda_{(1)}^{j}\right\}+\delta_{j k} q_{(3)}^{k}\left\{X^{i}, \lambda_{(2)}^{j}\right\}
\end{aligned}
$$

using the Dirac brackets of coordinates.

\subsection{Partial reduction I}

We start with Clèment Lagrangian (54) once more but, in this case, we will apply the partial reduction presented in (3.2.1). Here is the reduced first order Lagrangian derived from the Clèment Lagrangian

$$
L_{C_{1}}^{C}=-\frac{m \zeta}{2}\left\|\mathbf{q}_{(2)}\right\|^{2}+\frac{\zeta^{2}}{2 \mu m} \mathbf{q}_{(1)} \cdot \mathbf{q}_{(2)} \times \dot{\mathbf{q}}_{(2)}+\boldsymbol{\lambda}_{1} \cdot\left(\dot{\mathbf{q}}_{(1)}-\mathbf{q}_{(2)}\right),
$$

where we have employed the coordinate transformations $\mathbf{x}=\mathbf{q}_{(1)}, \dot{\mathbf{x}}=\mathbf{q}_{(2)}, \ddot{\mathbf{x}}=\dot{\mathbf{q}}_{(2)}$. Here, $\boldsymbol{\lambda}_{1}$ is a set of Lagrange multipliers. The Euler-Lagrange equations generated by $L_{C_{1}}^{C}$ is computed to be

$$
\dot{\boldsymbol{\lambda}}_{1}=\frac{\zeta^{2}}{2 \mu m} \mathbf{q}_{(2)} \times \dot{\mathbf{q}}_{(2)}, \quad \boldsymbol{\lambda}_{1}=-m \zeta \mathbf{q}_{(2)}-\frac{\zeta^{2}}{\mu m} \mathbf{q}_{(1)} \times \dot{\mathbf{q}}_{(2)}+\frac{\zeta^{2}}{2 \mu m} \mathbf{q}_{(2)} \times \dot{\mathbf{q}}_{(1)}, \quad \dot{\mathbf{q}}_{(1)}-\mathbf{q}_{(2)}=\mathbf{0} .
$$

We introduce the conjugate momenta $\left(\mathbf{p}^{(1)}, \mathbf{p}^{(2)}, \mathbf{p}^{\lambda_{1}}\right)$ dual to the velocities $\left(\dot{\mathbf{q}}_{(1)}, \dot{\mathbf{q}}_{(2)}, \dot{\boldsymbol{\lambda}}_{1}\right)$. The Legendre transformation leads to the following relationships

$$
\mathbf{p}^{(1)}=\boldsymbol{\lambda}_{1}, \quad \mathbf{p}^{(2)}=\frac{\zeta^{2}}{2 \mu m} \mathbf{q}_{(1)} \times \mathbf{q}_{(2)}, \quad \mathbf{p}^{\lambda_{1}}=0 .
$$


Since neither of the velocities $\dot{\mathbf{q}}_{(1)}, \dot{\mathbf{q}}_{(2)}$ and $\dot{\boldsymbol{\lambda}}_{1}$ can be solved from these relations, we introduce the primary constraints

$$
\boldsymbol{\Phi}^{(1)}=\mathbf{p}^{(1)}-\boldsymbol{\lambda}_{1}, \quad \mathbf{\Phi}^{(2)}=\mathbf{p}^{(2)}-\frac{\zeta^{2}}{2 \mu m} \mathbf{q}_{(1)} \times \mathbf{q}_{(2)}, \quad \mathbf{\Phi}^{\left(\lambda_{1}\right)}=\mathbf{p}^{\lambda_{1}} .
$$

In accordance with this, the total Hamiltonian function is defined to be

$$
H_{T}=H_{C_{1}}+\mathbf{u}_{(1)} \cdot \boldsymbol{\Phi}^{(1)}+\mathbf{u}_{(2)} \cdot \boldsymbol{\Phi}^{(2)}+\mathbf{u}_{\left(\lambda_{1}\right)} \cdot \boldsymbol{\Phi}^{\left(\lambda_{1}\right)},
$$

where $\mathbf{u}_{(1)}, \mathbf{u}_{(2)}$ and $\mathbf{u}_{\left(\lambda_{1}\right)}$ are Lagrange multipliers whereas $H_{C_{1}}$ is the canonical Hamiltonian function computed as

$$
H_{C_{1}}=\frac{m \zeta}{2}\left\|\mathbf{q}_{(2)}\right\|^{2}+\mathbf{p}^{(1)} \cdot \mathbf{q}_{(2)} .
$$

Here are the steps of the Dirac-Bergmann constraint algorithm.

Dirac-Bergmann constraint algorithm step 1: Consistency checks of each of the primary constraints exhibited in the equations (79) read

$$
\begin{aligned}
\dot{\boldsymbol{\Phi}}^{(1)} & =\left\{\boldsymbol{\Phi}^{(1)}, H_{T}\right\} \approx \frac{\zeta^{2}}{2 \mu m} \mathbf{q}_{(2)} \times \mathbf{u}_{(2)}-\mathbf{u}_{\left(\lambda_{1}\right)}, \\
\dot{\boldsymbol{\Phi}}^{(2)} & =\left\{\boldsymbol{\Phi}^{(2)}, H_{T}\right\} \approx m \zeta \mathbf{q}_{(2)}+\mathbf{p}^{(1)}-\frac{\zeta^{2}}{2 \mu m} \mathbf{u}_{(1)} \times \mathbf{q}_{(2)}+\frac{\zeta^{2}}{\mu m} \mathbf{u}_{(2)} \times \mathbf{q}_{(1)}, \\
\dot{\boldsymbol{\Phi}}^{\left(\lambda_{1}\right)} & =\left\{\boldsymbol{\Phi}^{\left(\lambda_{1}\right)}, H_{T}\right\} \approx \mathbf{u}_{(1)} .
\end{aligned}
$$

From these expressions, we determine the Lagrange multipliers $\mathbf{u}_{(1)} \approx 0$ and $\mathbf{u}_{\left(\lambda_{1}\right)} \approx \frac{\zeta^{2}}{2 \mu m} \mathbf{q}_{(2)} \times \mathbf{u}_{(2)}$. From Eq. (82), we can solve only two components of the $\mathbf{u}_{(2)}$. So that there remains a secondary constraint

$$
\Phi=\left(m \zeta \mathbf{q}_{(2)}+\mathbf{p}^{(1)}\right) \cdot \mathbf{q}_{(1)} .
$$

By adding this secondary constraint to the total Hamiltonian function $H_{T}$ in (80), we revise the Hamiltonian as $H_{T}^{1}=H_{T}+u \Phi$, where $u$ being a Lagrange multiplier.

Dirac-Bergmann constraint algorithm step 2: Consistency of the secondary constraint $\Phi$ can be checked by the following calculation

$$
\dot{\Phi}=\left\{\Phi, H_{T}^{1}\right\} \approx\left(m \zeta \mathbf{q}_{(1)}+\mathbf{p}^{(1)}\right) \cdot \mathbf{q}_{(2)}+\mathbf{u}_{(2)}\left(m \zeta \mathbf{q}_{(1)}+\frac{\zeta^{2}}{2 \mu m} \mathbf{q}_{(2)} \times \mathbf{q}_{(1)}\right) .
$$

Notice that, one may determine the third component of $\mathbf{u}_{(2)}$ using Eq.(84) whereas consistency equation of the constraint $\boldsymbol{\Phi}^{(2)}$ leads us to determine $u$. Hence, all Lagrange multipliers are computed to be

$$
\begin{aligned}
\mathbf{u}_{(1)} & \approx \mathbf{0} \\
\mathbf{u}_{(2)} & \approx \frac{\mu m}{\zeta^{2}\left\|\mathbf{q}_{(1)}\right\|^{2}} \mathbf{q}_{1} \times \mathbf{D}-\frac{3}{2 m \zeta \mathbf{q}_{(1)}^{2}}\left(\mathbf{D} \cdot \mathbf{q}_{(2)}\right) \mathbf{q}_{(1)}, \\
\mathbf{u}_{\left(\lambda_{1}\right)} & \approx \frac{\zeta^{2}}{2 \mu m} \mathbf{q}_{(2)} \times\left(\frac{\mu m}{\zeta^{2}\left\|\mathbf{q}_{(1)}\right\|^{2}} \mathbf{q}_{(1)} \times \mathbf{D}-\frac{3}{2 m \zeta\left\|\mathbf{q}_{(1)}\right\|^{2}}\left(\mathbf{D} \cdot \mathbf{q}_{(2)}\right) \mathbf{q}_{(1)}\right), \\
u & \approx-\frac{\Phi}{m \zeta\left\|\mathbf{q}_{(1)}\right\|^{2}}
\end{aligned}
$$

where we have used the abbreviation $\mathbf{D}=m \zeta \mathbf{q}_{(2)}+\mathbf{p}^{(1)}$. 
The total Hamiltonian function and the equations of motion: Substitutions of the Lagrange multipliers $u, \mathbf{u}_{(1)}, \mathbf{u}_{(2)}$ and $\mathbf{u}_{\left(\lambda_{1}\right)}$ into $H_{T}^{1}$ determines the total Hamiltonian function

$$
\begin{aligned}
H_{T}^{1} & =\frac{1}{2} \mathbf{p}^{(1)} \cdot \mathbf{q}_{(2)}+\frac{1}{2\left\|\mathbf{q}_{(1)}\right\|^{2}}\left(\mathbf{q}_{(1)} \cdot \mathbf{q}_{(2)}\right)\left(\mathbf{D} \cdot \mathbf{q}_{(1)}\right)+\frac{\mu m}{\zeta^{2}\left\|\mathbf{q}_{(1)}\right\|^{2}} \mathbf{p}^{(2)} \cdot \mathbf{q}_{(1)} \times \mathbf{D} \\
& -\frac{3}{2 m \zeta\left\|\mathbf{q}_{(1)}\right\|^{2}}\left(\mathbf{D} \cdot \mathbf{q}_{(2)}\right)\left(\mathbf{q}_{(1)} \cdot \mathbf{p}^{(2)}\right)+\frac{1}{2\left\|\mathbf{q}_{(1)}\right\|^{2}} \mathbf{p}^{\lambda_{1}} \cdot \mathbf{q}_{(2)} \times\left(\mathbf{q}_{(1)} \times \mathbf{D}\right) \\
& -\frac{3 \zeta}{4 \mu m^{2}\left\|\mathbf{q}_{(1)}\right\|^{2}} \mathbf{p}^{\lambda_{1}} \cdot \mathbf{q}_{(2)} \times \mathbf{q}_{(1)}\left(\mathbf{D} \cdot \mathbf{q}_{(2)}\right)-\frac{\left(\mathbf{D} \cdot \mathbf{q}_{(1)}\right)^{2}}{m \zeta\left\|\mathbf{q}_{(1)}\right\|^{2}}-\frac{\Phi^{2}}{m \zeta\left\|\mathbf{q}_{(1)}\right\|^{2}} .
\end{aligned}
$$

The Hamilton's equations generated by the total Hamiltonian function (866) are

$$
\begin{aligned}
\dot{\mathbf{q}}_{(1)} & \approx \frac{1}{2} \mathbf{q}_{(2)}+\frac{1}{2\left\|\mathbf{q}_{(1)}\right\|^{2}}\left(\mathbf{q}_{(1)} \cdot \mathbf{q}_{(2)}\right) \mathbf{q}_{(1)}+\frac{\mu m}{\zeta^{2}\left\|\mathbf{q}_{1}\right\|^{2}} \mathbf{p}^{(2)} \times \mathbf{q}_{(1)} \\
\dot{\mathbf{q}}_{(2)} & \approx \frac{\mu m}{\zeta^{2}\left\|\mathbf{q}_{(1)}\right\|^{2}} \mathbf{q}_{(1)} \times \mathbf{D}-\frac{3}{2 m \zeta\left\|\mathbf{q}_{(1)}\right\|^{2}}\left(\mathbf{D} \cdot \mathbf{q}_{(2)}\right) \mathbf{q}_{(1)} \\
\dot{\boldsymbol{\lambda}}_{1} & \approx \frac{1}{2\left\|\mathbf{q}_{(1)}\right\|^{2}} \mathbf{q}_{(2)} \times\left(\mathbf{q}_{(1)} \times \mathbf{D}\right)-\frac{3 \zeta}{4 \mu m^{2}\left\|\mathbf{q}_{(1)}\right\|^{2}} \mathbf{q}_{(2)} \times \mathbf{q}_{(1)}\left(\mathbf{D} \cdot \mathbf{q}_{(2)}\right) \\
\dot{\mathbf{p}}^{(1)} & \approx \frac{-1}{2\left\|\mathbf{q}_{(1)}\right\|^{2}}\left(\mathbf{q}_{(1)} \cdot \mathbf{q}_{(2)}\right) \mathbf{D}-\frac{\mu m}{\zeta^{2} \mathbf{q}_{(1)}^{2}} \mathbf{D} \times \mathbf{p}^{(2)}+\frac{3}{2 m \zeta\left\|\mathbf{q}_{(1)}\right\|^{2}} \mathbf{p}^{(2)}\left(\mathbf{D} \cdot \mathbf{q}_{(2)}\right) \\
& +\frac{2 \mu m}{\zeta^{2}\left\|\mathbf{q}_{(1)}\right\|^{4}}\left(\mathbf{p}^{(2)} \cdot \mathbf{q}_{(1)} \times \mathbf{D}\right) \mathbf{q}_{(1)} \\
\dot{\mathbf{p}}^{(2)} & \approx-\frac{1}{2} \mathbf{p}^{(1)}-\frac{m \zeta}{2\left\|\mathbf{q}_{(1)}\right\|^{2}}\left(\mathbf{q}_{(1)} \cdot \mathbf{q}_{(2)}\right) \mathbf{q}_{(1)}-\frac{\mu m^{2}}{\zeta\left\|\mathbf{q}_{(1)}\right\|^{2}} \mathbf{p}^{(2)} \times \mathbf{q}_{(1)} \\
\dot{\mathbf{p}}^{\lambda_{1}} & \approx 0
\end{aligned}
$$

The equation of motion for $\mathbf{p}^{(1)}$ gives the Euler-Lagrange equations (56) whereas the remaining ones are identically satisfied after the back substitution of the momenta.

The Dirac bracket: We shall derive the Hamilton's equations using the Dirac bracket. To do this, we record here the set of second class constraints

$$
\boldsymbol{\Phi}^{(1)} \equiv \mathbf{p}^{(1)}-\boldsymbol{\lambda}_{1}, \quad \boldsymbol{\Phi}^{(2)} \equiv \mathbf{p}^{(2)}-\frac{\zeta^{2}}{2 \mu m} \mathbf{q}_{(1)} \times \mathbf{q}_{(2)}, \quad \boldsymbol{\Phi}^{\left(\lambda_{1}\right)} \equiv \mathbf{p}^{\lambda_{1}}, \quad \Phi \equiv\left(m \zeta \mathbf{q}_{(2)}+\mathbf{p}^{(1)}\right) \cdot \mathbf{q}_{(1)} .
$$

In the present case, the Dirac bracket (27) turns out to be

$$
\begin{aligned}
\{F, G\}_{D B} & =\{F, G\}-\left\{F, \Phi_{n}^{(1)}\right\} C_{1 \lambda}^{n n^{\prime}}\left\{\delta_{n^{\prime} l} \Phi_{\left(\lambda_{1}\right)}^{l}, G\right\}-\left\{F, \Phi_{n}^{(2)}\right\} C_{11}^{n n^{\prime}}\left\{\Phi_{n^{\prime}}^{(2)}, G\right\} \\
& -\left\{F, \Phi_{n}^{(2)}\right\} C_{1 \lambda}^{n n^{\prime}}\left\{\delta_{n^{\prime} l} \Phi_{\lambda}^{l}, G\right\}-\left\{F, \Phi_{n}^{(2)}\right\} C_{1 \Phi}^{n}\{\Phi, G\} \\
& -\left\{F, \delta_{n l} \Phi_{\lambda}^{l}\right\} C_{\lambda 0}^{n n^{\prime}}\left\{\Phi_{n^{\prime}}^{(0)}, G\right\}-\left\{F, \delta_{n l} \Phi_{\lambda}^{l}\right\} C_{\lambda_{1} 1}^{n n^{\prime}}\left\{\Phi_{n^{\prime}}^{(1)}, G\right\} \\
& -\left\{F, \delta_{n l} \Phi_{\lambda}^{l}\right\} C_{\lambda \lambda}^{n n^{\prime}}\left\{\delta_{n^{\prime} r} \Phi_{n}^{r}, G\right\}-\left\{F, \delta_{n l} \phi_{\lambda}^{l}\right\} C_{\lambda \Phi}^{n}\{\Phi, G\} \\
& -\{F, \Phi\} C_{\Phi 1}^{n^{\prime}}\left\{\Phi_{n^{\prime}}^{(1)}, G\right\}-\{F, \Phi\} M_{\Phi 1}^{n^{\prime}}\left\{\delta_{n^{\prime} l} \Phi_{\lambda}^{l}, G\right\} .
\end{aligned}
$$

Here, the matrix $C$ is computed to be

$$
C=\left(\begin{array}{cccc}
0 & \zeta^{2} \epsilon_{n^{\prime} n k^{\prime}} q_{(2)}^{k^{\prime}} / 2 \mu m & -\delta_{n}^{n^{\prime}} & -D_{n} \\
-\zeta^{2} \epsilon_{n n^{\prime} k^{\prime}} q_{(2)}^{k^{\prime}} / 2 \mu m & \zeta^{2} \epsilon_{n n^{\prime} k} q_{(1)}^{k} / \mu m & 0 & -E_{n} \\
\delta_{n^{\prime}}^{n} & 0 & 0 & 0 \\
D_{n^{\prime}} & E_{n^{\prime}} & 0 & 0
\end{array}\right)
$$


with determinant $\zeta^{6}\left\|q_{(1)}\right\|^{2} / \mu^{2}$. The inverse of $C$ is

$$
\begin{aligned}
C^{-1} & =\left(\begin{array}{cccc}
C_{00}^{n n^{\prime}} & C_{01}^{n n^{\prime}} & C_{0 \lambda}^{n n^{\prime}} & C_{0 \Phi}^{n} \\
C_{10}^{n n^{\prime}} & C_{11}^{n n^{\prime}} & C_{1 \lambda}^{n n^{\prime}} & C_{1 \Phi}^{n} \\
C_{\lambda 0}^{n n^{\prime}} & C_{\lambda 1}^{n n^{\prime}} & C_{\lambda \lambda}^{n n^{\prime}} & C_{\lambda \Phi}^{n} \\
C_{\Phi 0}^{n^{\prime}} & C_{\Phi 1}^{n^{\prime}} & C_{\Phi \lambda}^{n^{\prime}} & C_{\Phi \Phi}
\end{array}\right) \\
& =\left(\begin{array}{cccc}
0 & 0 & \delta^{n n^{\prime}} & 0 \\
0 & -\mu \epsilon^{n n^{\prime} k} E_{k} / \zeta^{3}\left\|\mathbf{q}_{(1)}\right\|^{2} & A^{n n^{\prime}} & q_{(1)}^{n} / m \zeta\left\|\mathbf{q}_{(1)}\right\|^{2} \\
-\delta^{n n^{\prime}} & B^{n n^{\prime}} & C_{k r s}^{n k} q_{(1)}^{r} q_{(2)}^{s} \\
0 & -q_{(1)}^{n^{\prime}} / m \zeta\left\|\mathbf{q}_{(1)}\right\|^{2} & \zeta \epsilon_{k r s} \delta^{n^{\prime} k} q_{(1)}^{r} q_{(1)}^{s} / 2 m^{2} \mu\left\|\mathbf{q}_{(1)}^{2}\right\| & 0 \\
2 m^{2} \mu\left\|\mathbf{q}_{(1)}\right\| \|^{2}
\end{array}\right)
\end{aligned}
$$

where

$$
A_{n^{\prime}}^{n}=\frac{1}{2 m \zeta\left\|\mathbf{q}_{(1)}\right\|^{2}} \epsilon^{n r k} \epsilon_{n^{\prime} r l} q_{(2)}^{l} E_{k}+\frac{1}{m \zeta\left\|\mathbf{q}_{(1)}\right\|^{2}} q_{1}^{n} D_{n^{\prime}}, \quad B^{n n^{\prime}}=-\frac{\epsilon_{r k p} \delta^{n k} q_{(2)}^{p} \epsilon^{r n^{\prime} s} E_{s}}{2 m \zeta\left\|\mathbf{q}_{(1)}\right\|^{2}}+\frac{D^{n} q_{(1)}^{n^{\prime}}}{\left\|\mathbf{q}_{(1)}\right\|^{2}}
$$

The Dirac brackets of the coordinates are

$$
\begin{aligned}
& \left\{q_{(1)}^{i}, q_{(2)}^{j}\right\}_{D B}=-\frac{q_{(1)}^{i} q_{(1)}^{j}}{m \zeta\left\|\mathbf{q}_{(1)}\right\|^{2}}, \\
& \left\{q_{(1)}^{i}, \lambda_{1}^{j}\right\}_{D B}=\delta_{i j}-\frac{q_{(1)}^{i} q_{(1)}^{j}}{m \zeta\left\|\mathbf{q}_{(1)}\right\|^{2}}, \\
& \left\{q_{(1)}^{i}, p_{j}^{(1)}\right\}_{D B}=\delta_{j}^{i}-\frac{\zeta \epsilon_{j k^{\prime} n^{\prime}} q_{(1)}^{n^{\prime}} q_{(2)}^{k^{\prime}}}{2 m^{2} \mu\left\|\mathbf{q}_{(1)}\right\|^{2}} \\
& \left\{q_{(2)}^{i}, q_{(2)}^{j}\right\}_{D B}=-\frac{\mu \epsilon^{i j k} E_{k}}{\zeta^{3}\left\|\mathbf{q}_{(1)}\right\|^{2}}, \\
& \left\{q_{(2)}^{i}, \lambda_{1}^{j}\right\}_{D B}=A^{i j}, \\
& \left\{q_{(2)}^{i}, p_{j}^{(1)}\right\}_{D B}=-\frac{1}{2 m \zeta\left\|\mathbf{q}_{(1)}\right\|^{2}} \epsilon^{i r k} \epsilon_{j r l} q_{(2)}^{l} E_{k}-\frac{q_{(1)}^{i} D_{j}}{m \zeta\left\|\mathbf{q}_{(1)}\right\|^{2}}, \\
& \left\{q_{(2)}^{i}, p_{j}^{(2)}\right\}_{D B}=\delta_{j}^{i}-\frac{1}{2 m \zeta\left\|\mathbf{q}_{(1)}\right\|^{2}} \epsilon^{i n^{\prime} k} \epsilon_{j n^{\prime} k^{\prime}} q_{(1)}^{k^{\prime}} E_{k}-\frac{q_{(1)}^{i} \delta_{j l} q_{(1)}^{l}}{\left\|\mathbf{q}_{(1)}\right\|^{2}}, \\
& \left\{\lambda_{1}^{i}, \lambda_{1}^{j}\right\}_{D B}=C^{i j} \\
& \left\{\lambda_{1}^{i}, p_{j}^{(1)}\right\}_{D B}=\frac{\zeta^{2}}{2 \mu m} B^{i r} \epsilon_{j k r q_{(2)}^{k}}+\frac{\zeta}{2 m^{2} \mu\left\|\mathbf{q}_{(1)}\right\|^{2}} \delta^{i k} \epsilon_{k r s} q_{(1)}^{r} q_{(2)}^{s} D_{j} \\
& \left\{\lambda_{1}^{i}, p_{j}^{(2)}\right\}_{D B}=\frac{\zeta^{2}}{2 \mu m} B^{i r} \epsilon_{j r k} q_{(1)}^{k}+\frac{\zeta^{2}}{2 m \mu\left\|\mathbf{q}_{(1)}\right\|^{2}} \epsilon_{i r s} q_{(1)}^{r} q_{(2)}^{s}\left(\delta_{j l} q_{(1)}^{l}\right) \\
& \left\{p_{i}^{(1)}, p_{j}^{(1)}\right\}_{D B}=\frac{\zeta \epsilon_{i k^{\prime} j} q_{(2)}^{k^{\prime}} E_{k} q_{(2)}^{k}}{4 \mu m^{2}\left\|\mathbf{q}_{(1)}\right\|^{2}}-\frac{\zeta \epsilon_{i k n} q_{(2)}^{k} q_{(1)}^{n} D_{j}}{2 \mu m^{2}\left\|\mathbf{q}_{(1)}\right\|^{2}}+\frac{\zeta \epsilon_{j k^{\prime} n^{\prime}} q_{(2)}^{k^{\prime}} q_{(1)}^{n^{\prime}} D_{i}}{2 \mu m^{2}\left\|\mathbf{q}_{(1)}\right\|^{2}} \\
& \left\{p_{i}^{(1)}, p_{j}^{(2)}\right\}_{D B}=-\frac{\zeta \epsilon_{i k^{\prime} j} q_{(2)}^{k^{\prime}} D_{i} q_{(1)}^{k}}{4 \mu m^{2}\left\|\mathbf{q}_{(1)}\right\|^{2}}+\frac{\zeta \epsilon_{i k^{\prime} n} q_{(2)}^{k^{\prime}} q_{(1)}^{n} E_{j}}{4 \mu m^{2}\left\|\mathbf{q}_{(1)}\right\|^{2}} \\
& \left\{p_{i}^{(2)}, p_{j}^{(2)}\right\}_{D B}=-\frac{\zeta}{4 \mu m^{3}\left\|\mathbf{q}_{(1)}\right\|^{2}} \epsilon_{i n k^{\prime}} q_{(1)}^{k^{\prime}} \epsilon^{n r k} E_{k} \epsilon_{j r l} q_{(1)}^{l}
\end{aligned}
$$

and all the others are zero. Here we used abbreviations

$$
D_{i}=m \zeta \delta_{i j} q_{(2)}^{j}+p_{i}^{(1)}, \quad E_{i}=m \zeta \delta_{i j} q_{(1)}^{j}+p_{i}^{(2)}, \quad \delta_{i j} q_{(1)}^{i} q_{(1)}^{j}=\left\|\mathbf{q}_{(1)}\right\|^{2} .
$$

Calculation of the Hamilton's equations of motion using the Dirac bracket of coordinates is a matter of a direct calculation. 


\subsubsection{Unconstrained Variational Formalism}

Now, we shall present the case of unconstrained Lagrangian in this framework. We point out that, Dirac analysis of this realization is much more simple. Let us start with unconstraint Lagrangian (42) corresponding to the first order Clèment Lagrangian (76) which is

$$
L_{U_{1}}^{C}=\frac{m \zeta}{2} \mathbf{q}_{(2)} \cdot \mathbf{q}_{(2)}-m \zeta \mathbf{q}_{(2)} \cdot \dot{\mathbf{q}}_{(1)}-\frac{\zeta^{2}}{2 \mu m} \mathbf{q}_{(1)} \cdot \mathbf{q}_{(2)} \times \dot{\mathbf{q}}_{(2)}+\frac{\zeta^{2}}{\mu m} \mathbf{q}_{(1)} \cdot \dot{\mathbf{q}}_{(1)} \times \dot{\mathbf{q}}_{(2)}
$$

by substituting $\boldsymbol{\lambda}_{1}$ in (77) into $L_{C_{1}}^{C}$ in (76). The conjugate momenta turn out to be

$$
\mathbf{p}^{(1)}=-m \zeta \mathbf{q}_{(2)}-\frac{\zeta^{2}}{\mu m} \mathbf{q}_{(1)} \times \dot{\mathbf{q}}_{(2)}, \quad \mathbf{p}^{(2)}=-\frac{\zeta^{2}}{2 \mu m} \mathbf{q}_{(1)} \times \mathbf{q}_{(2)}+\frac{\zeta^{2}}{\mu m} \mathbf{q}_{(1)} \times \dot{\mathbf{q}}_{(1)} .
$$

whereas the canonical Hamiltonian function for the first order unconstraint Lagrangian (93) is

$$
\begin{aligned}
H_{U_{1}} & =-\frac{m \zeta}{2}\left\|\mathbf{q}_{(2)}\right\|^{2}+\frac{\mu m}{\zeta^{2} q_{(1)}^{3}}\left(p_{2}^{(2)} D_{1}-p_{1}^{(2)} D_{2}\right)+\frac{1}{2 q_{(1)}^{3}}\left(-D_{2}\left(q_{(1)}^{2} q_{(2)}^{3}-q_{(1)}^{3} q_{(2)}^{2}\right)\right. \\
& \left.+D_{1}\left(q_{(1)}^{3} q_{(2)}^{1}-q_{(1)}^{1} q_{(2)}^{3}\right)\right)
\end{aligned}
$$

Here, we are still using the abbreviation $D_{i}=p_{i}^{(1)}+m \zeta \delta_{i j} q_{(1)}^{j}$. Equations (94) lead to solve two components of $\dot{\mathbf{q}}_{(1)}$ and two components of $\dot{\mathbf{q}}_{(2)}$. The rest determines a set of primary constraints

$$
\Phi=\mathbf{p}^{(2)} \cdot \mathbf{q}_{(1)}, \quad \phi=\left(\mathbf{p}^{(1)}+m \zeta \mathbf{q}_{(2)}\right) \cdot \mathbf{q}_{(1)} .
$$

Then the total Hamiltonian function is defined to be

$$
H_{T}=H_{U_{1}}+U \Phi+V \phi
$$

with Lagrange multipliers $U$ and $V$. Here are the steps of the Dirac-Bergmann constraint algorithm and the computation of the total Hamiltonian function.

Dirac-Bergmann constraint algorithm step 1: Consistency checks of the primary constraints $\Phi$ and $\phi$ give no more constraint, instead we can determine $U$ and $V$ as

$$
U \approx \frac{\frac{\mu m^{2}}{\zeta q_{(1)}^{3}}\left(q_{(1)}^{2} D_{1}+q_{(1)}^{1} D_{2}\right)-\frac{3}{2} D_{i} q_{(2)}^{i}}{m \zeta\left\|\mathbf{q}_{(\mathbf{1})}\right\|^{2}}, \quad V \approx \frac{\delta_{i j} q_{(1)}^{i} q_{(2)}^{j}-\frac{2 \mu m}{\zeta^{2} q_{(1)}^{3}}\left(p_{2}^{(1)} q_{(1)}^{1}+p_{1}^{(1)} q_{(1)}^{2}\right)}{\left\|\mathbf{q}_{(\mathbf{1})}\right\|^{2}} .
$$

Substitutions of $U$ and $V$ into (97) result with the determination of the total Hamiltonian function $H_{T}$ for the first order unconstraint Lagrangian (93). 
The equations of motion: In this case, the Hamilton's equations of motion are

$$
\begin{aligned}
& \dot{q}_{(1)}^{i} \approx \frac{\mu m}{\zeta^{2} q_{(1)}^{3}}\left(p_{2}^{(2)} \delta_{1}^{i}-p_{1}^{(2)} \delta_{2}^{i}\right)+\frac{1}{2 q_{(1)}^{3}}\left(-\delta_{2}^{i}\left(q_{(1)}^{2} q_{(2)}^{3}-q_{(1)}^{3} q_{(2)}^{2}\right)+\delta_{1}^{i}\left(q_{(1)}^{3} q_{(2)}^{1}-q_{(1)}^{1} q_{(2)}^{3}\right)\right) \\
& +\left(\frac{\delta_{k j} q_{(1)}^{k} q_{(2)}^{j}-\frac{2 \mu m}{\zeta^{2} q_{(1)}^{3}}\left(p_{2}^{(1)} q_{(1)}^{1}+p_{1}^{(1)} q_{(1)}^{2}\right)}{\left\|\mathbf{q}_{(\mathbf{1})}\right\|^{2}}\right) q_{(1)}^{i}, \\
& \dot{q}_{(2)}^{i} \approx \frac{\mu m}{\zeta^{2} q_{(1)}^{3}}\left(\delta_{2}^{i} D_{1}-\delta_{1}^{i} D_{2}\right)+\left(\frac{\frac{\mu m^{2}}{\zeta q_{0}^{3}}\left(q_{(1)}^{2} D_{1}+q_{(1)}^{1} D_{2}\right)-\frac{3}{2} D_{i} q_{(2)}^{i}}{m \zeta\left\|\mathbf{q}_{(1)}\right\|^{2}}\right) q_{(1)}^{i}, \\
& \dot{p}_{i}^{(1)} \approx \frac{-1}{2 q_{(1)}^{3}}\left(-D_{2}\left(\delta_{i}^{2} q_{(2)}^{3}-\delta_{i}^{3} q_{(2)}^{2}\right)+D_{1}\left(\delta_{i}^{3} q_{(2)}^{1}-\delta_{i}^{1} q_{(2)}^{3}\right)\right)+\frac{\mu m}{\zeta^{2}\left(q_{(1)}^{3}\right)^{2}} \delta_{i}^{3}\left(p_{2}^{(1)} D_{1}-p_{1}^{(1)} D_{2}\right) \\
& -\left(\frac{\frac{\mu m^{2}}{\zeta q_{(1)}^{3}}\left(q_{(1)}^{2} D_{1}+q_{(1)}^{1} D_{2}\right)-\frac{3}{2} D_{i} q_{(2)}^{i}}{m \zeta\left\|\mathbf{q}_{(1)}\right\|^{2}}\right) p_{i}^{(1)}-\left(\frac{\delta_{i j} q_{(1)}^{i} q_{(2)}^{j}-\frac{2 \mu m}{\zeta^{2} q_{(1)}^{3}}\left(p_{2}^{(1)} q_{(1)}^{1}+p_{1}^{(1)} q_{(1)}^{2}\right)}{\left\|\mathbf{q}_{(\mathbf{1})}\right\|^{2}}\right) D_{i} \\
& +\frac{1}{2\left(q_{(1)}^{3}\right)^{2}} \delta_{i}^{3}\left(-D_{2}\left(q_{(1)}^{2} q_{(2)}^{3}-q_{(1)}^{3} q_{(2)}^{2}\right)+D_{1}\left(q_{(1)}^{3} q_{(2)}^{1}-q_{(1)}^{1} q_{(2)}^{3}\right)\right), \\
& \dot{p}_{i}^{(2)} \approx \frac{\mu m^{2}}{\zeta q_{(1)}^{3}}\left(p_{2}^{(1)} \delta_{i}^{(1)}+p_{1}^{(1)} \delta_{i}^{2}\right)+m \zeta \delta_{i j} q_{(2)}^{j}-\frac{m \zeta}{2 q_{(1)}^{3}}\left(\delta_{i}^{2}\left(q_{(1)}^{2} q_{(2)}^{3}-q_{(1)}^{3} q_{(2)}^{2}\right)+\delta_{i}^{1}\left(q_{(1)}^{3} q_{(2)}^{1}-q_{(1)}^{1} q_{(2)}^{3}\right)\right) \\
& -\frac{1}{2 q_{(1)}^{3}}\left(-D_{2}\left(\delta_{i}^{3} q_{(1)}^{2}-\delta_{i}^{2} q_{(1)}^{3}\right)+D_{1}\left(\delta_{i}^{1} q_{(1)}^{3}-\delta_{i}^{3} q_{(1)}^{1}\right)\right) \\
& -\left(\frac{\delta_{i j} q_{(1)}^{i} q_{(2)}^{j}-\frac{2 \mu m}{\zeta^{2} q_{(1)}^{3}}\left(p_{2}^{(1)} q_{(1)}^{1}+p_{1}^{(1)} q_{(1)}^{2}\right)}{\left\|\mathbf{q}_{(\mathbf{1})}\right\|^{2}}\right) m \zeta q_{(1)}^{i} .
\end{aligned}
$$

Here, the equations governing the momenta $\mathbf{p}^{(1)}$ give the Euler-Lagrange equations (56) , and the remaining equations are identically satisfied.

The Dirac bracket: Let us find the Hamilton's equations for the unconstraint Lagrangian (93) using the Dirac algebra. All constraints are second class since their Poisson bracket

$$
\{\Phi, \phi\}=-m \zeta\left\|\mathbf{q}_{(1)}\right\|^{2}
$$

is nonzero. In this case the matrix $M$ in the definition of the Dirac bracket given in (27) has a relatively simple form given by

$$
M=\left(\begin{array}{cc}
\{\Phi, \Phi\} & \{\Phi, \phi\} \\
\{\phi, \Phi\} & \{\phi, \phi\}
\end{array}\right)=m \zeta\left\|\mathbf{q}_{(1)}\right\|^{2}\left(\begin{array}{cc}
0 & -1 \\
1 & 0
\end{array}\right)
$$

In accordance with this, we compute the Dirac bracket of two functions as follows

$$
\{F, G\}_{D B}=\{F, G\}-\frac{1}{m \zeta\left\|\mathbf{q}_{(1)}\right\|^{2}}(\{F, \Phi\}\{\phi, G\}-\{F, \phi\}\{\Phi, G\}) .
$$


More explicitly, the Dirac brackets of the coordinates are

$$
\begin{aligned}
& \left\{q_{(1)}^{i}, q_{(2)}^{j}\right\}_{D B}=-\frac{1}{m \zeta\left\|\mathbf{q}_{(1)}\right\|^{2}} q_{(1)}^{i} q_{(2)}^{j} \\
& \left\{q_{(1)}^{i}, p_{j}^{(1)}\right\}_{D B}=\frac{1}{m \zeta\left\|\mathbf{q}_{(1)}\right\|^{2}} q_{(1)}^{i} p_{j}^{(2)}+\delta_{j}^{i} \\
& \left\{q_{(2)}^{i}, p_{j}^{(1)}\right\}_{D B}=-\frac{1}{m \zeta\left\|\mathbf{q}_{(1)}\right\|^{2}} q_{(1)}^{i}\left(p_{j}^{(1)}+m \zeta \delta_{j k} q_{(2)}^{k}\right) \\
& \left\{q_{(2)}^{i}, p_{j}^{(2)}\right\}_{D B}=\delta_{j}^{i}-\frac{1}{\left\|\mathbf{q}_{(1)}\right\|^{2}} q_{(1)}^{i} q_{(1)}^{j} \\
& \left.\left\{p_{i}^{(1)}, p_{j}^{(1)}\right\}_{D B}=\frac{1}{m \zeta\left\|\mathbf{q}_{(1)}\right\|^{2}} p_{i}^{(2)}\left(p_{j}^{(1)}+m \zeta \delta_{j k} q_{(2)}^{k}\right)-\left(p_{i}^{(1)}+m \zeta \delta_{i k} q_{(2)}^{k}\right) p_{j}^{(2)}\right] \\
& \left\{p_{i}^{(1)}, p_{j}^{(2)}\right\}_{D B}=\frac{1}{\left\|\mathbf{q}_{(1)}\right\|^{2}} p_{i}^{(2)} \delta_{j k} q_{(2)}^{k}
\end{aligned}
$$

and all the rest are zero. It is now straight forward to compute the Hamilton's equations using the Dirac brackets.

\subsection{Partial reduction II}

Starting with Clèment Lagrangian (54), and following the definition in (43), we introduce the following reduced Lagrangian

$$
L_{C_{2}}^{C}=-\frac{m \zeta}{2}\left\|\dot{\mathbf{q}}_{(1)}\right\|^{2}+\frac{\zeta^{2}}{2 \mu m} \mathbf{q}_{(1)} \cdot \dot{\mathbf{q}}_{(1)} \times \dot{\mathbf{q}}_{(2)}+\boldsymbol{\lambda}_{2} \cdot\left(\dot{\mathbf{q}}_{(1)}-\mathbf{q}_{(2)}\right)
$$

using coordinate transformations $\mathbf{x}=\mathbf{q}_{(1)}, \dot{\mathbf{x}}=\dot{\mathbf{q}}_{(1)}=\mathbf{q}_{(2)}, \ddot{\mathbf{x}}=\dot{\mathbf{q}}_{(2)}$ and a set $\boldsymbol{\lambda}_{2}$ of Lagrange multipliers. EulerLagrange equations are

$$
\dot{\boldsymbol{\lambda}}_{2}=m \zeta \ddot{\mathbf{q}}_{1}+\frac{\zeta^{2}}{\mu m} \dot{\mathbf{q}}_{(1)} \times \dot{\mathbf{q}}_{(2)}-\frac{\zeta^{2}}{2 \mu m} \ddot{\mathbf{q}}_{(2)} \times \mathbf{q}_{(1)}, \quad \boldsymbol{\lambda}_{2}=-\frac{\zeta^{2}}{2 \mu m} \mathbf{q}_{(1)} \times \ddot{\mathbf{q}}_{(1)}, \quad \dot{\mathbf{q}}_{(1)}-\mathbf{q}_{(2)}=\mathbf{0} .
$$

Before to pass Hamiltonian formalism for $L_{C_{2}}^{C}$ given in (104), let us rewrite the Lagrangian in (104) as

$$
L_{C_{2}}^{C}=-\frac{m \zeta}{2}\left\|\dot{\mathbf{q}}_{(1)}\right\|^{2}+\frac{\zeta^{2}}{2 \mu m} \mathbf{q}_{(1)} \cdot \dot{\mathbf{q}}_{(1)} \times \dot{\mathbf{q}}_{(2)}+\left[\boldsymbol{\lambda}_{1}+m \zeta \dot{\mathbf{q}}_{1}-\frac{\zeta^{2}}{2 \mu m} \dot{\mathbf{q}}_{(2)} \times \mathbf{q}_{(1)}\right] \cdot\left(\dot{\mathbf{q}}_{(1)}-\mathbf{q}_{(2)}\right)
$$

using the relation between the Lagrange multipliers

$$
\boldsymbol{\lambda}_{2}=\boldsymbol{\lambda}_{1}+m \zeta \dot{\mathbf{q}}_{(1)}-\frac{\zeta^{2}}{2 \mu m} \dot{\mathbf{q}}_{(2)} \times \mathbf{q}_{(1)} .
$$

After some cancellation, the Lagrangian function (106) turns out to be

$$
L_{C_{2}}^{C}=\frac{m \zeta}{2}\left\|\dot{\mathbf{q}}_{(1)}\right\|^{2}+\frac{\zeta^{2}}{2 \mu m} \mathbf{q}_{(1)} \cdot \mathbf{q}_{(2)} \times \dot{\mathbf{q}}_{(2)}-m \zeta \dot{\mathbf{q}}_{(1)} \cdot \mathbf{q}_{(2)}+\boldsymbol{\lambda}_{1} \cdot\left(\dot{\mathbf{q}}_{(1)}-\mathbf{q}_{(2)}\right) .
$$

In order to pass Hamiltonian formalism for $L_{C_{2}}^{C}$ in (107), the conjugate momenta are defined by

$$
\mathbf{P}^{(1)}=m \zeta \dot{\mathbf{q}}_{(1)}+\boldsymbol{\lambda}_{1}-m \zeta \mathbf{q}_{(2)}, \quad \mathbf{P}^{(2)}=\frac{\zeta^{2}}{2 \mu m} \mathbf{q}_{(1)} \times \mathbf{q}_{(2)}, \quad \mathbf{P}^{\lambda_{2}}=0 .
$$

From these momenta it is possible to solve $\dot{\mathbf{q}}_{(1)}$. The remaining momenta lead to primary constraints

$$
\mathbf{\Phi}=\mathbf{P}^{(2)}-\frac{\zeta^{2}}{2 \mu m} \mathbf{q}_{(1)} \times \mathbf{q}_{(2)}, \quad \mathbf{\Phi}^{\lambda_{2}}=\mathbf{P}^{\lambda_{2}} .
$$


For the Lagrangian $L_{C_{2}}^{C}$, the canonical Hamiltonian function (39) is

$$
H=\frac{1}{2 m \zeta}\left\|\mathbf{P}^{(1)}-\boldsymbol{\lambda}_{1}\right\|^{2}+\mathbf{q}_{(2)} \cdot \mathbf{P}^{(1)}+\frac{m \zeta}{2}\left\|\mathbf{q}_{(2)}\right\|^{2}
$$

thus total Hamiltonian function is

$$
H_{T}=H+\mathbf{U} \cdot \boldsymbol{\Phi}+\mathbf{U}_{\lambda} \boldsymbol{\Phi}^{\lambda_{2}}
$$

with the sets $\mathbf{U}$ and $\mathbf{V}$ of Lagrange multipliers. Here are the steps of the Dirac-Bergmann Constraint algorithm and the computation of the total Hamiltonian function.

Dirac-Bergmann constraint algorithm step 1: Consistency of $\boldsymbol{\Phi}$ and $\boldsymbol{\Phi}^{\lambda_{2}}$ lead to

$$
\begin{aligned}
\dot{\boldsymbol{\Phi}} & =\left\{\boldsymbol{\Phi}, H_{T}\right\}=-\mathbf{P}^{1}-m \zeta \mathbf{q}_{(2)}+\frac{\zeta}{2 \mu m^{2}} \mathbf{q}_{(2)} \times\left(\mathbf{P}^{\mathbf{1}}-\boldsymbol{\lambda}_{1}\right)+\frac{\zeta^{2}}{\mu m} \mathbf{U} \times \mathbf{q}_{(1)} \\
\dot{\boldsymbol{\Phi}}^{\lambda_{2}} & =\left\{\boldsymbol{\Phi}^{\lambda_{2}}, H_{T}\right\}=\frac{1}{m \zeta}\left(\mathbf{P}^{1}-\boldsymbol{\lambda}_{1}\right) .
\end{aligned}
$$

So that we arrive at the secondary constraints $\phi=\left(\mathbf{P}^{1}+m \zeta \mathbf{q}_{(2)}\right) \cdot \mathbf{q}_{(1)}$ and $\beta=\mathbf{P}^{1}-\boldsymbol{\lambda}_{1}$.

Dirac-Bergmann constraint algorithm step 2: From the conservations of these secondary constraints no more constraint arise and the Lagrange multipliers $\mathbf{U}$ and $\mathbf{U}_{\lambda}$ are determined. All constraints derived for $L_{C_{2}}^{C}$ are completely equivalent to $L_{C_{1}}^{C}$. Also Hamiltonian function for both Lagrangians are identical. That means Hamiltonian formalism for two Lagrangian system $L_{C_{1}}^{C}$ and $L_{C_{2}}^{C}$ are equivalent.

\begin{tabular}{|c|c|c|}
\hline & Partial Reduction I & Partial Reduction II \\
\hline Reduction & $\mathbf{x}=\mathbf{q}_{(1)}, \dot{\mathbf{x}}=\mathbf{q}_{(2)}, \quad \ddot{\mathbf{x}}=\dot{\mathbf{q}}_{(2)}$ & $\mathbf{x}=\mathbf{q}_{(1)}, \dot{\mathbf{x}}=\dot{\mathbf{q}}_{(1)}, \quad \ddot{\mathbf{x}}=\dot{\mathbf{q}}_{(2)}$ \\
\hline Coordinates & $\mathbf{q}_{(1)}, \mathbf{q}_{(2)}, \boldsymbol{\lambda}_{1}, \mathbf{p}^{(1)}, \mathbf{p}^{(2)}, \mathbf{p}^{\left(\lambda_{1}\right)}$ & $\mathbf{q}_{(1)}, \dot{\mathbf{q}}_{(1)}, \boldsymbol{\lambda}_{2}, \mathbf{P}^{(1)}, \mathbf{P}^{(2)}, \mathbf{p}^{\left(\lambda_{2}\right)}$ \\
\hline Primary Constraints & $\begin{aligned} \boldsymbol{\Phi}^{(1)} & =\mathbf{p}^{(1)}-\boldsymbol{\lambda}_{1} \\
\boldsymbol{\Phi}^{(2)} & =\mathbf{p}^{(2)}-\frac{\zeta^{2}}{2 \mu m} \mathbf{q}_{(1)} \times \mathbf{q}_{(2)} \\
\boldsymbol{\Phi}^{\left(\lambda_{1}\right)} & =\mathbf{p}^{\lambda_{1}}\end{aligned}$ & $\begin{aligned} \boldsymbol{\Phi}^{(2)} & =\mathbf{P}^{(2)}-\frac{\zeta^{2}}{2 \mu m} \mathbf{q}_{(1)} \times \mathbf{q}_{(2)} \\
\boldsymbol{\Phi}^{\left(\lambda_{2}\right)} & =\mathbf{P}^{\lambda_{2}}\end{aligned}$ \\
\hline Secondary Constraints & $\mathbf{\Phi}=\left(\mathbf{p}^{(1)}+m \zeta \mathbf{q}_{(2)}\right) \cdot \mathbf{q}_{(1)}$ & $\begin{aligned} \boldsymbol{\Phi} & =\left(\mathbf{P}^{(1)}+m \zeta \mathbf{q}_{(2)}\right) \cdot \mathbf{q}_{(1)} \\
\boldsymbol{\Phi}^{(1)} & =\mathbf{P}^{(1)}-\boldsymbol{\lambda}_{1}\end{aligned}$ \\
\hline
\end{tabular}

Remark 1 All constraints derived for the Lagrangian $L_{C_{2}}^{C}$ in Eq. 104) are completely identical with the constraints in (88) which are derived for the Lagrangian $L_{C_{1}}^{C}$ in Eq. (76). Notice the following table comparing the constraints derived for the partial reductions I and II:

So the constraints are almost the same for two methods. This gives that the Dirac bracket for the method of Partial Reduction II will be the same with the Dirac bracket for the method of Partial Reduction I.

Remark 2 Unconstraint variational formalism is useful if the Lagrange multipliers does not contain second order derivatives. Notice that, the Lagrange multipliers presented in (105) contain second order derivatives. So that, it is not feasible to study the unconstraint formalism in this present case.

\subsection{Deriglazov's trick / Schmidt's method}

This is the last method for the reduction of the Clèment Lagrangian (54) into the first order formalism. In this subsection, we shall employ Deriglazov's trick / Schmidt's method presented in subsection (3.3.2). By referring to the reduced Lagrangian function in Eq.(53), we introduce the following first order Lagrangian function

$$
L_{A}^{C}=-\frac{m \zeta}{2}\|\dot{\mathbf{x}}\|^{2}+\frac{\zeta^{2}}{2 \mu m} \mathbf{x} \cdot \dot{\mathbf{x}} \times \mathbf{s}-\dot{\gamma} \cdot \dot{\mathbf{x}}-\gamma \cdot \mathbf{s}
$$


using the coordinate transformations

$$
\ddot{\mathbf{x}}=\mathbf{s}, \quad \gamma=\frac{\partial L_{A}^{C}}{\partial \mathbf{s}}=\frac{\zeta^{2}}{2 \mu m} \mathbf{x} \times \dot{\mathbf{x}} .
$$

Here, the Lagrangian $L_{A}^{C}$ depends on the velocity components $(\dot{\mathbf{x}}, \dot{\mathbf{s}}, \dot{\gamma})$. This Lagrangian is degenerate. In the dual picture, the conjugate momenta are $\left(\mathbf{p}^{(x)}, \mathbf{p}^{(s)}, \mathbf{p}^{(\gamma)}\right)$. The Legendre transformation reads the following definitions for the momenta

$$
\mathbf{p}^{(x)}=-m \zeta \dot{\mathbf{x}}+\frac{\zeta^{2}}{2 \mu m} \mathbf{s} \times \mathbf{x}-\dot{\gamma}, \quad \mathbf{p}^{(\gamma)}=-\dot{\mathbf{x}}, \quad \mathbf{p}^{(s)}=0 .
$$

From these relationships, it is possible to solve velocities $\dot{\mathrm{x}}$ and $\dot{\gamma}$ in terms of the momenta as follows

$$
\dot{\mathbf{x}}=-\mathbf{p}^{(\gamma)}, \quad \dot{\gamma}=m \zeta \mathbf{p}^{(\gamma)}+\frac{\zeta^{2}}{2 \mu m} \mathbf{s} \times \mathbf{x}-\mathbf{p}^{(x)} .
$$

We cannot solve the velocity $\dot{\mathbf{s}}$ in terms of the momenta, this induces a primary constraint $\varphi^{s}=\mathbf{p}^{(s)}=0$ in the cotangent bundle.

Let us write the canonical Hamiltonian function for the first order Lagrangian (113) as

$$
\begin{aligned}
H & =\mathbf{p}^{(x)} \cdot \dot{\mathbf{x}}+\mathbf{p}^{(\gamma)} \cdot \dot{\gamma}+\mathbf{p}^{(s)} \cdot \dot{\mathbf{s}}-L_{A}^{C} \\
& =-\mathbf{p}^{(x)} \cdot \mathbf{p}^{(\gamma)}+\frac{m \zeta}{2}\left\|\mathbf{p}^{(\gamma)}\right\|^{2}+\frac{\zeta^{2}}{2 \mu m} \mathbf{p}^{(\gamma)} \cdot \mathbf{s} \times \mathbf{x}+\gamma \cdot \mathbf{s} .
\end{aligned}
$$

We introduce the total Hamiltonian function by adding the primary constraints into the canonical Hamiltonian function. So that we have

$$
H_{T}=H+\mathbf{v}_{s} \cdot \varphi^{s},
$$

Here, $\mathbf{v}_{s}$ denoted a set of Lagrange multipliers. Here are the steps of the Dirac-Bergmann constraint algorithm and the explicit computation of the total Hamiltonian function.

Dirac-Bergmann constraint algorithm step 1: Consistencies of the primary constraints $\varphi^{s}$

$$
\dot{\varphi}^{s}=\left\{\boldsymbol{\varphi}^{s}, H_{T}\right\} \approx-\frac{\zeta^{2}}{2 \mu m} \mathbf{x} \times \mathbf{p}^{(\gamma)}-\gamma
$$

lead to a set of secondary constraints $\varphi=\frac{\zeta^{2}}{2 \mu m} \mathbf{x} \times \mathbf{p}^{(\gamma)}+\gamma$. The total Hamiltonian should be revised as $H_{T}^{1}=$ $H_{T}+\mathbf{v} \cdot \varphi$.

Dirac-Bergmann constraint algorithm step 2: Consistency of these secondary constraints

$$
\dot{\varphi}=\left\{\boldsymbol{\varphi}, H_{T}^{1}\right\} \approx \frac{\zeta^{2}}{2 \mu m} \mathbf{s} \times \mathbf{x}-\mathbf{p}^{(x)}+m \zeta \mathbf{p}^{(\gamma)}+\frac{\zeta^{2}}{\mu m} \mathbf{v} \times \mathbf{x}
$$

result with the determination of the Lagrange multiplier $\mathbf{v}$ but one more constraint $\psi=\mathbf{x} \cdot\left(\mathbf{p}^{(\mathbf{x})}-\mathbf{m} \zeta \mathbf{p}^{(\gamma)}\right)$ arise by taking the dot product of the Eq. (119) by $\mathbf{x}$. From the conservation of $\psi$, the Lagrange multipliers $\mathbf{v}$ are determined. Thus the Eq.(119) gives a set of tertiary constraints

$$
\mathbf{\Phi}=\frac{\zeta^{2}}{2 \mu m} \mathbf{s} \times \mathbf{x}-\mathbf{p}^{(x)}+m \zeta \mathbf{p}^{(\gamma)}
$$

Revision of the total Hamiltonian function result with $H_{T}^{2}=H_{T}^{1}+\mathbf{w} \cdot \mathbf{\Phi}$

Dirac-Bergmann constraint algorithm step 3: For the tertiary constraints, we compute

$$
\dot{\boldsymbol{\Phi}}=\left\{\mathbf{\Phi}, H_{T}^{2}\right\} \approx \frac{3 \zeta^{2}}{2 \mu m} \mathbf{p}^{(\gamma)} \times \mathbf{s}-m \zeta \mathbf{s}+\frac{\zeta^{2}}{2 \mu m} \mathbf{v}_{s} \times \mathbf{x}+\frac{2 \zeta^{2}}{\mu m} \mathbf{w} \times \mathbf{s}
$$


and for the secondary and primary constraints, we get

$$
\dot{\varphi}=\left\{\boldsymbol{\varphi}, H_{T}^{2}\right\} \approx \boldsymbol{\varphi}+m \zeta \mathbf{w}-\frac{\zeta^{2}}{\mu \mathbf{m}} \mathbf{w} \times \mathbf{p}^{(\gamma)}, \quad \dot{\varphi} \mathbf{s}=\left\{\varphi^{\mathbf{s}}, \mathbf{H}_{\mathbf{T}}^{\mathbf{2}}\right\} \approx \boldsymbol{\varphi}+\frac{\zeta^{\mathbf{2}}}{\mu \mathbf{m}} \mathbf{w} \times \mathbf{x} .
$$

These equations determine $\mathbf{w} \approx \mathbf{0}$ and substitution of $\mathbf{w}$ into (120) leads to the determination of two components of $\mathbf{v}_{s}$, and a new constraint

$$
\chi=\left(\frac{3 \zeta^{2}}{2 \mu m} \mathbf{p}^{(\gamma)} \times \mathbf{s}-m \zeta \mathbf{s}\right) \cdot \mathbf{x}
$$

which can also be computed by taking dot product of (120) with $\mathbf{x}$. Now, total Hamiltonian function becomes $H_{T}^{3}=H_{T}^{2}+z \chi$.

Dirac-Bergmann constraint algorithm step 4: Finally from the conservation of $\chi$ we can determine third component of $\mathbf{v}_{s}$ and no more constraint arise. Thus $\mathbf{v}_{s}$ is determined as

$$
\mathbf{v}_{s} \approx \frac{\frac{\mu m^{2}}{\zeta}\left(\mathbf{s} \cdot \mathbf{p}^{(\gamma)}\right) \mathbf{x}+\frac{\mu m}{\zeta^{2}}\left(\frac{3 \zeta^{2}}{2 \mu m} \mathbf{x} \times \mathbf{p}^{(\gamma)}-m \zeta \mathbf{x}\right) \times\left(\frac{3 \zeta^{2}}{2 \mu m} \mathbf{p}^{(\gamma)} \times \mathbf{s}-m \zeta \mathbf{s}\right)}{m \zeta\|\mathbf{x}\|^{2}} .
$$

We summarize the results of computations with acceleration bundle in the following table.

\begin{tabular}{|c|c|}
\hline & Deriglazov/Schmidt's Method \\
\hline Reduction & $\ddot{\mathbf{x}}=\mathbf{s}, \gamma=\frac{\zeta^{2}}{2 \mu m} \mathbf{x} \times \dot{\mathbf{x}}$, \\
\hline Coordinates & $\mathbf{x}, \gamma, \boldsymbol{s}, \mathbf{p}^{(x)}, \mathbf{p}^{(\gamma)}, \mathbf{p}^{(s)}$ \\
\hline Primary Constraints & $\boldsymbol{\varphi}^{s}=\mathbf{p}^{(s)}$ \\
\hline Secondary Constraints & $\boldsymbol{\varphi}=\frac{\zeta^{2}}{2 \mu m} \mathbf{x} \times \mathbf{p}^{(\gamma)}+\gamma$ \\
\hline Tertiary Constraints & $\mathbf{\Phi}=m \zeta \mathbf{p}^{(\gamma)}+\frac{\zeta^{2}}{2 \mu m} \mathbf{s} \times \mathbf{x}-\boldsymbol{p}^{(x)}$ \\
\hline Quaternary Constraints & $\chi=\mathbf{x} \cdot\left(m \zeta \mathbf{s}+\frac{3 \zeta^{2}}{2 \mu m} \mathbf{s} \times \mathbf{p}^{(\gamma)}\right)$ \\
\hline
\end{tabular}

The total Hamiltonian function and the equations of motion: Substitution of $\mathbf{v}_{s}$ into (117) results with the explicit expression of the total Hamiltonian function

$$
\begin{aligned}
H_{T} & =-\mathbf{p}^{(x)} \cdot \mathbf{p}^{(\gamma)}+\frac{m \zeta}{2}\left(\mathbf{p}^{(\gamma)}\right)^{2}+\frac{\zeta^{2}}{2 \mu m} \mathbf{p}^{(\gamma)} \cdot \mathbf{s} \times \mathbf{x}+\gamma \cdot \mathbf{x} \\
& +\left[\frac{\frac{\mu m^{2}}{\zeta}\left(\mathbf{s} \cdot \mathbf{p}^{(\gamma)}\right) \mathbf{x}+\frac{\mu m}{\zeta^{2}}\left(\frac{3 \zeta^{2}}{2 \mu m} \mathbf{x} \times \mathbf{p}^{(\gamma)}-m \zeta \mathbf{x}\right) \times\left(\frac{3 \zeta^{2}}{2 \mu m} \mathbf{p}^{(\gamma)} \times \mathbf{s}-m \zeta \mathbf{s}\right)}{m \zeta\|\mathbf{x}\|^{2}}\right] \cdot \boldsymbol{p}^{s} .
\end{aligned}
$$

In accordance with this, we compute the Hamilton's equations of motion as follows

$$
\begin{aligned}
\dot{\mathbf{x}} & \approx-\mathbf{p}^{(\gamma)} \\
\dot{\gamma} & \approx-\mathbf{p}^{(x)}+m \zeta \mathbf{p}^{(\gamma)}+\frac{\zeta^{2}}{2 \mu m} \mathbf{s} \times \mathbf{x} \\
\dot{\boldsymbol{s}} & \approx \frac{\frac{\mu m^{2}}{\zeta}\left(\mathbf{s} \cdot \mathbf{p}^{(\gamma)}\right) \mathbf{x}+\frac{\mu m}{\zeta^{2}}\left(\frac{3 \zeta^{2}}{2 \mu m} \mathbf{x} \times \mathbf{p}^{(\gamma)}-m \zeta \mathbf{x}\right) \times\left(\frac{3 \zeta^{2}}{2 \mu m} \mathbf{p}^{(\gamma)} \times \mathbf{s}-m \zeta \mathbf{s}\right)}{m \zeta|| \mathbf{x} \|^{2}} \\
\dot{\mathbf{p}}^{(x)} & \approx-\frac{\zeta^{2}}{2 \mu m} \mathbf{p}^{(\gamma)} \times \mathbf{s}, \\
\dot{\mathbf{p}}^{(\gamma)} & \approx-\boldsymbol{s}, \\
\dot{\mathbf{p}}^{(s)} & \approx-\frac{\zeta^{2}}{2 \mu m} \mathbf{x} \times \mathbf{p}^{(\gamma)}-\gamma .
\end{aligned}
$$

The Hamilton's equations governing the motion of $\mathbf{p}^{(x)}$ gives Euler-Lagrange equations in (56), and the remaining equations identically satisfied after a back substitution of the momenta in terms of the velocities. 
Remark 3 An alternative reduction of the the second order Clèment Lagrangian (54) can be achieved in the following way. Define the first order Lagrangian

$$
L_{T}^{C}=-\frac{m \zeta}{2}\left\|\dot{\mathbf{q}}_{(1)}\right\|^{2}-\frac{\zeta^{2}}{2 \mu m} \dot{\mathbf{q}}_{(1)} \cdot \dot{\mathbf{q}}_{(2)}+\boldsymbol{\tau} \cdot\left(\mathbf{q}_{(1)} \times \dot{\mathbf{q}}_{(1)}-\mathbf{q}_{(2)}\right)
$$

using action angle coordinates $\mathbf{x}=\mathbf{q}_{(1)}$ and $\mathbf{x} \times \dot{\mathbf{x}}=\mathbf{q}_{(2)}$. Here, $\boldsymbol{\tau}$ is a set of Lagrange multipliers. See that the Lagrangian (125) is the same with the one in (113) after employing the identifications $\zeta^{2} \mathbf{s} / 2 \mu m=\tau$ and $\zeta^{2} \mathbf{q}_{(2)} / 2 \mu m=\gamma$.

\section{$5 \quad$ Sarığlu-Tekin Lagrangian}

We start with a 6 -dimensinal manifold $Q$ with local coordinates $(\mathbf{x}, \mathbf{y})$ consisting of two 3 -dimensional vectors. The higher order tangent bundles are equipped with the following induced sets of coordinates

$$
\begin{aligned}
(\mathbf{x}, \mathbf{y}, \dot{\mathbf{x}}, \dot{\mathbf{y}}) & \in T Q \\
(\mathbf{x}, \mathbf{y}, \dot{\mathbf{x}}, \dot{\mathbf{y}}, \ddot{\mathbf{x}}, \ddot{\mathbf{y}}) & \in T^{2} Q \\
(\mathbf{x}, \mathbf{y}, \dot{\mathbf{x}}, \dot{\mathbf{y}}, \ddot{\mathbf{x}}, \ddot{\mathbf{y}}, \dddot{\mathbf{x}}, \dddot{\mathbf{y}}) & \in T^{3} Q .
\end{aligned}
$$

In [64], Sarıoğlu and Tekin proposed a degenerate second order Lagrangian on $T^{2} Q$ given by

$$
L^{S T}[\mathbf{x}, \mathbf{y}]=\frac{1}{2}\left[a\left(\dot{\mathbf{x}}^{2}+\dot{\mathbf{y}}^{2}\right)+\frac{2}{\mu} \dot{\mathbf{y}} \cdot \ddot{\mathbf{x}}-m^{2}\left(\mathbf{y}^{2}+\mathbf{x}^{2}\right)\right] .
$$

In this case, the Euler-Lagrange equations (18) take the particular form

$$
m^{2} \mathbf{x}+a \ddot{\mathbf{x}}=\frac{1}{\mu} \mathbf{y}^{(3)}, \quad m^{2} \mathbf{y}+a \ddot{\mathbf{y}}=-\frac{1}{\mu} \mathbf{x}^{(3)} .
$$

\subsection{Total Reduction}

At first, we are applying the total reduction method exhibited in the subsection (3.1) to the Sarığlu and Tekin Lagrangian (127) in order to arrive at a first order formalism. Accordingly, writing the Lagrangian function defined in (31) for the the Sarığlu and Tekin Lagrangian, we compute the following particular form

$$
L_{C}^{S T}=\frac{1}{2}\left[a\left(\mathbf{q}_{(1)}^{2}+\mathbf{q}_{(3)}^{2}\right)+\frac{2}{\mu} \mathbf{q}_{(3)} \cdot \mathbf{q}_{(2)}-m^{2}\left(\mathbf{y}^{2}+\mathbf{x}^{2}\right)\right]+\boldsymbol{\lambda}_{1} \cdot\left(\dot{\mathbf{x}}-\mathbf{q}_{(1)}\right)+\boldsymbol{\lambda}_{2} \cdot\left(\dot{\mathbf{q}}_{(1)}-\mathbf{q}_{(2)}\right)+\boldsymbol{\lambda}_{3} \cdot\left(\dot{\mathbf{y}}-\mathbf{q}_{(3)}\right)
$$

using coordinate transformations $\dot{\mathbf{x}}=\mathbf{q}_{(1)}, \dot{\mathbf{q}}_{(1)}=\mathbf{q}_{(2)}, \dot{\mathbf{y}}=\mathbf{q}_{(3)}$ and the Lagrange multipliers $\boldsymbol{\lambda}_{1}, \boldsymbol{\lambda}_{2}, \boldsymbol{\lambda}_{3}$. The conjugate momenta for (129) are computed as

$$
\mathbf{p}^{(1)}=\boldsymbol{\lambda}_{2}, \quad \mathbf{p}^{(2)}=0, \quad \mathbf{p}^{(3)}=0, \quad \mathbf{p}^{\left(\lambda_{1}\right)}=0, \quad \mathbf{p}^{\left(\lambda_{\mathbf{2}}\right)}=\mathbf{0}, \quad \mathbf{p}^{\left(\lambda_{\mathbf{3}}\right)}=\mathbf{0}, \quad \mathbf{p}^{(\mathbf{x})}=\boldsymbol{\lambda}_{\mathbf{1}}, \quad \mathbf{p}^{(\mathbf{y})}=\boldsymbol{\lambda}_{\mathbf{3}} .
$$

It is not possible to solve the velocities in terms of the momenta instead we have the following set of primary constraints

$$
\begin{gathered}
\boldsymbol{\Phi}^{(1)}=\mathbf{p}^{(1)}-\lambda_{2}, \quad \Phi^{(2)}=\mathbf{p}^{(2)}, \quad \boldsymbol{\Phi}^{(3)}=\mathbf{p}^{(3)}, \quad \boldsymbol{\Phi}^{(x)}=\mathbf{p}^{(x)}-\boldsymbol{\lambda}_{1}, \quad \boldsymbol{\Phi}^{(y)}=\mathbf{p}^{(y)}-\boldsymbol{\lambda}_{3} \\
\boldsymbol{\Phi}^{\left(\lambda_{1}\right)}=\mathbf{p}^{\left(\lambda_{1}\right)}, \quad \boldsymbol{\Phi}^{\left(\lambda_{2}\right)}=\mathbf{p}^{\left(\lambda_{2}\right)}, \quad \boldsymbol{\Phi}^{\left(\lambda_{3}\right)}=\mathbf{p}^{\left(\lambda_{3}\right)} .
\end{gathered}
$$

The canonical Hamiltonian function is

$$
H=-\frac{1}{2}\left[a\left(\mathbf{q}_{(1)}^{2}+\mathbf{q}_{(3)}^{2}\right)+\frac{2}{\mu} \mathbf{q}_{(3)} \cdot \mathbf{q}_{(2)}-m^{2}\left(\mathbf{y}^{2}+\mathbf{x}^{2}\right)\right]+\boldsymbol{\lambda}_{1} \cdot \mathbf{q}_{1}+\boldsymbol{\lambda}_{2} \cdot \mathbf{q}_{2}+\boldsymbol{\lambda}_{3} \cdot \mathbf{q}_{3}
$$

and the total Hamiltonian function is

$$
H_{T}=H+\mathbf{U}_{1} \cdot \Phi^{(1)}+\mathbf{U}_{2} \cdot \Phi^{(2)}+\mathbf{U}_{3} \cdot \Phi^{(3)}+\mathbf{U}_{\lambda_{1}} \Phi^{\left(\lambda_{1}\right)}+\mathbf{U}_{\lambda_{2}} \cdot \Phi^{\left(\lambda_{2}\right)}+\mathbf{U}_{\lambda_{3}} \cdot \Phi^{\left(\lambda_{3}\right)}+\mathbf{U}_{x} \cdot \boldsymbol{\Phi}^{(x)}+\mathbf{U}_{y} \cdot \boldsymbol{\Phi}^{(y)} .
$$


Here are the steps of the Dirac-Bergmann constraint algorithm and the computation of the total Hamiltonian function.

Dirac-Bergmann constraint algorithm step 1: Conservations of primary constraints

$$
\begin{aligned}
\dot{\boldsymbol{\Phi}}^{(1)} & =\left\{\boldsymbol{\Phi}^{(1)}, H_{T}\right\} \approx a \mathbf{q}_{(1)}-\boldsymbol{\lambda}_{1}-\mathbf{U}_{\lambda_{2}}, \quad \dot{\boldsymbol{\Phi}}^{(2)}=\left\{\boldsymbol{\Phi}^{(2)}, H_{T}\right\} \approx \frac{1}{\mu} \mathbf{q}_{(3)}-\boldsymbol{\lambda}_{2}, \\
\dot{\boldsymbol{\Phi}}^{(3)} & =\left\{\boldsymbol{\Phi}^{(3)}, H_{T}\right\} \approx a \mathbf{q}_{(3)}+\frac{1}{\mu} \mathbf{q}_{(2)}-\boldsymbol{\lambda}_{3}, \quad \dot{\boldsymbol{\Phi}}^{\left(\lambda_{1}\right)}=\left\{\boldsymbol{\Phi}^{\left(\lambda_{1}\right)}, H_{T}\right\} \approx-\mathbf{q}_{(1)}+\mathbf{U}_{x}, \\
\dot{\boldsymbol{\Phi}}^{\left(\lambda_{2}\right)} & =\left\{\boldsymbol{\Phi}^{\left(\lambda_{2}\right)}, H_{T}\right\} \approx \mathbf{U}_{1}-\mathbf{q}_{(2)}, \quad \dot{\boldsymbol{\Phi}}^{\left(\lambda_{3}\right)}=\left\{\boldsymbol{\Phi}^{\left(\lambda_{3}\right)}, H_{T}\right\} \approx \mathbf{U}_{y}-\mathbf{q}_{(3)} \\
\dot{\boldsymbol{\Phi}}^{(x)} & =\left\{\boldsymbol{\Phi}^{(x)}, H_{T}\right\} \approx-m^{2} \mathbf{x}-\mathbf{U}_{\lambda_{1}}, \quad \dot{\boldsymbol{\Phi}}^{(y)}=\left\{\boldsymbol{\Phi}^{(y)}, H_{T}\right\} \approx-m^{2} \mathbf{y}-\mathbf{U}_{\lambda_{3}}
\end{aligned}
$$

lead to the determinations of $\mathbf{U}_{1}, \mathbf{U}_{x}, \mathbf{U}_{y}, \mathbf{U}_{\lambda_{1}}, \mathbf{U}_{\lambda_{2}}, \mathbf{U}_{\lambda_{3}}$ and there arise the following secondary constraints

$$
\mathbf{\Phi}=\frac{1}{\mu} \mathbf{q}_{(3)}-\boldsymbol{\lambda}_{2}, \quad \phi=a \mathbf{q}_{(3)}+\frac{1}{\mu} \mathbf{q}_{(2)}-\boldsymbol{\lambda}_{3} .
$$

In accordance with this, by substitutions of the Lagrange multipliers and addition of secondary constraints we revise the total Hamiltonian function as

$$
\begin{aligned}
H_{T}^{1} & =H_{T}+\mathbf{U} \cdot \mathbf{\Phi}+\mathbf{V} \cdot \boldsymbol{\phi} \\
& =-\frac{1}{2}\left[a\left(\mathbf{q}_{(1)}^{2}+\mathbf{q}_{(3)}^{2}\right)+\frac{2}{\mu} \mathbf{q}_{(3)} \cdot \mathbf{q}_{(2)}-m^{2}\left(\mathbf{y}^{2}+\mathbf{x}^{2}\right)\right]+\boldsymbol{p}^{1} \cdot \mathbf{q}_{2}-m^{2}\left(\mathbf{p}^{\left(\lambda_{1}\right)} \cdot \mathbf{x}+\mathbf{p}^{\left(\lambda_{3}\right)} \cdot \mathbf{y}\right) \\
& +\mathbf{p}^{\left(\lambda_{2}\right)} \cdot\left[a \mathbf{q}_{(1)}-\boldsymbol{\lambda}_{1}\right]+\mathbf{p}^{y} \cdot \mathbf{q}_{(3)}+\mathbf{U}_{2} \cdot \boldsymbol{\Phi}^{2}+\mathbf{U}_{3} \cdot \boldsymbol{\Phi}^{3}+\mathbf{U} \cdot \boldsymbol{\Phi}+\mathbf{V} \cdot \boldsymbol{\phi} .
\end{aligned}
$$

\begin{tabular}{|c|c|}
\hline & Total Reduction \\
\hline Reduction & $\dot{\mathbf{x}}=\mathbf{q}_{(1)}, \ddot{\mathbf{x}}=\mathbf{q}_{(2)}, \dot{\mathbf{y}}=\mathbf{q}_{(3)}$, \\
\hline Coordinates & $\mathbf{x}, \mathbf{y}, \mathbf{q}_{(1)}, \mathbf{q}_{(2)}, \mathbf{q}_{(3)}, \boldsymbol{\lambda}_{1}, \boldsymbol{\lambda}_{2}, \boldsymbol{\lambda}_{3}, \mathbf{p}^{x}, \mathbf{p}^{y}, \mathbf{p}^{(1)}, \mathbf{p}^{(2)}, \mathbf{p}^{(3)}, \mathbf{p}^{\left(\lambda_{1}\right)}, \mathbf{p}^{\left(\lambda_{2}\right)}, \mathbf{p}^{\left(\lambda_{3}\right)}$ \\
\hline Primary Constraints & 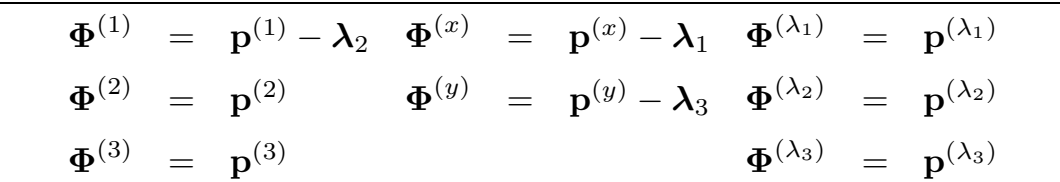 \\
\hline Secondary constraints & $\boldsymbol{\Phi}=\frac{1}{\mu} \mathbf{q}_{(3)}-\boldsymbol{\lambda}_{2}, \quad \phi=a \mathbf{q}_{(\mathbf{3})}+\frac{1}{\mu} \mathbf{q}_{(\mathbf{2})}-\boldsymbol{\lambda}_{\mathbf{3}}$ \\
\hline
\end{tabular}

Dirac-Bergmann constraint algorithm step 2: Consistency checks of the secondary constraints $\boldsymbol{\Phi}$ and $\phi$ are

$$
\dot{\boldsymbol{\Phi}}=\left\{\boldsymbol{\Phi}, H_{T}^{1}\right\} \approx \frac{1}{\mu} \mathbf{U}_{3}-a \mathbf{q}_{(1)}+\boldsymbol{\lambda}_{1}, \quad \dot{\boldsymbol{\phi}}=\left\{\boldsymbol{\phi}, H_{T}^{1}\right\} \approx a \mathbf{U}_{3}+\frac{1}{\mu} \mathbf{U}_{2}+m^{2} \mathbf{y}
$$

These calculations determine the Lagrange multipliers $\mathbf{U}_{2}$ and $\mathbf{U}_{3}$. Conservation of $\boldsymbol{\Phi}^{\left(\lambda_{2}\right)}$ and $\boldsymbol{\Phi}^{\left(\lambda_{3}\right)}$ give the Lagrange multipliers $\mathbf{U} \approx \mu\left(\boldsymbol{\phi}-\mathbf{\Phi}^{\mathbf{y}}\right) \approx \mathbf{0}$ and $\mathbf{V} \approx \mu\left(\boldsymbol{\Phi}^{\mathbf{1}}-\mathbf{\Phi}\right) \approx \mathbf{0}$. Here is the table of constraints:

The total Hamiltonian function and the equations of motion: Substitutions of the Lagrange multipliers into (136) give the total Hamiltonian function

$$
\begin{aligned}
H_{T}^{1}= & -\frac{1}{2}\left[a\left(\mathbf{q}_{(1)}^{2}+\mathbf{q}_{(3)}^{2}\right)+\frac{2}{\mu} \mathbf{q}_{(3)} \cdot \mathbf{q}_{(2)}-m^{2}\left(\mathbf{y}^{2}+\mathbf{x}^{2}\right)\right]+\mathbf{p}^{(1)} \cdot \mathbf{q}_{2} \\
& -\mu m^{2} \mathbf{p}^{(2)} \cdot \mathbf{y}(a \mu-1)+\mu \mathbf{p}^{(3)} \cdot\left(-\boldsymbol{\lambda}_{1}+a \mathbf{q}_{(1)}\right)-m^{2} \mathbf{p}^{\left(\lambda_{1}\right)} \cdot \mathbf{x}+\mathbf{p}^{\left(\lambda_{2}\right)} \cdot\left(a \mathbf{q}_{(1)}-\boldsymbol{\lambda}_{1}\right) \\
& -m^{2} \mathbf{p}^{\left(\lambda_{3}\right)} \cdot \mathbf{y}+\mathbf{p}^{x} \cdot \mathbf{q}_{(1)}+\mathbf{p}^{y} \cdot \mathbf{q}_{(3)}+\mu \boldsymbol{\Phi} \cdot\left(\boldsymbol{\phi}-\boldsymbol{\Phi}^{y}\right)+\mu \boldsymbol{\phi} \cdot\left(\boldsymbol{\Phi}^{1}-\boldsymbol{\Phi}\right) .
\end{aligned}
$$


Accordingly, the Hamilton's equations are computed to be

$$
\begin{aligned}
\dot{\mathbf{q}}_{(1)} & \approx \mathbf{q}_{(2)}, \quad \dot{\mathbf{q}}_{(2)} \approx-a \mu^{2} m^{2} \mathbf{y}+\mu m^{2} \mathbf{y}, \quad \dot{\mathbf{q}}_{(3)} \approx-\mu\left(\boldsymbol{\lambda}_{1}-a \mathbf{q}_{(1)}\right), \quad \dot{\mathbf{x}} \approx \mathbf{q}_{(1)}, \quad \dot{\mathbf{y}} \approx \mathbf{q}_{(3)}, \\
\dot{\boldsymbol{\lambda}_{1}} & \approx-m^{2} \mathbf{x}, \quad \dot{\boldsymbol{\lambda}_{2}} \approx a \mathbf{q}_{(1)}-\boldsymbol{\lambda}_{1}, \quad \dot{\boldsymbol{\lambda}_{3}} \approx-m^{2} \mathbf{y}, \\
\dot{\mathbf{p}}^{(1)} & \approx a \mathbf{q}_{(1)}-\mathbf{p}^{x}-a \mathbf{p}^{\lambda_{2}}, \quad \dot{\mathbf{p}}^{(2)} \approx \frac{\mathbf{1}}{\mu} \mathbf{q}_{(\mathbf{3})}-\mathbf{p}^{(\mathbf{1})}, \quad \dot{\mathbf{p}}^{(\mathbf{3})} \approx \mathbf{a} \mathbf{q}_{(\mathbf{3})}+\frac{\mathbf{1}}{\mu} \mathbf{q}_{(\mathbf{2})}-\mathbf{p}^{\mathbf{y}}, \\
\dot{\mathbf{p}}^{\left(\lambda_{1}\right)} & \approx 0, \quad \dot{\mathbf{p}}^{\left(\lambda_{2}\right)} \approx 0, \quad \dot{\mathbf{p}}^{\left(\lambda_{3}\right)} \approx 0, \quad \dot{\mathbf{p}}^{x} \approx-m^{2} \mathbf{x}, \quad \dot{\mathbf{p}}^{y} \approx-m^{2} \mathbf{y},
\end{aligned}
$$

with the constraints $\mathbf{p}^{(2)}=\mathbf{p}^{(3)}=\mathbf{p}^{\left(\lambda_{2}\right)}=\mathbf{p}^{\left(\lambda_{3}\right)}=0$.

The Dirac bracket: All constraints for the first order Lagrangian (129)

$$
\begin{gathered}
\boldsymbol{\Phi}^{(1)}=\mathbf{p}^{(1)}-\boldsymbol{\lambda}_{2}, \quad \boldsymbol{\Phi}^{(x)}=\mathbf{p}^{(x)}-\boldsymbol{\lambda}_{1}, \quad \boldsymbol{\Phi}^{\left(\lambda_{1}\right)}=\mathbf{p}^{\left(\lambda_{1}\right)}, \quad \boldsymbol{\Phi}^{(2)}=\mathbf{p}^{(2)}, \quad \boldsymbol{\Phi}^{(y)}=\mathbf{p}^{(y)}-\boldsymbol{\lambda}_{3}, \\
\boldsymbol{\Phi}^{\left(\lambda_{2}\right)}=\mathbf{p}^{\left(\lambda_{2}\right)}, \quad \boldsymbol{\Phi}^{(3)}=\mathbf{p}^{(3)}, \quad \boldsymbol{\Phi}^{\left(\lambda_{3}\right)}=\mathbf{p}^{\left(\lambda_{3}\right)}, \quad \boldsymbol{\Phi}=\frac{1}{\mu} \mathbf{q}_{(3)}-\boldsymbol{\lambda}_{2}, \quad \boldsymbol{\phi}=a \mathbf{q}_{(\mathbf{3})}+\frac{\mathbf{1}}{\mu} \mathbf{q}_{(\mathbf{2})}-\boldsymbol{\lambda}_{\mathbf{3}}
\end{gathered}
$$

are second class since their bracket is nonzero. Poisson brackets of constraint defined in (26) becomes

$$
\mathbf{C}=\left(\begin{array}{cccccccccc}
\mathbf{0} & \mathbf{0} & \mathbf{0} & \mathbf{0} & -\delta_{i j} & \mathbf{0} & \mathbf{0} & \mathbf{0} & \mathbf{0} & \mathbf{0} \\
\mathbf{0} & \mathbf{0} & \mathbf{0} & \mathbf{0} & \mathbf{0} & \mathbf{0} & \mathbf{0} & \mathbf{0} & \mathbf{0} & -\frac{1}{\mu} \delta_{i j} \\
\mathbf{0} & \mathbf{0} & \mathbf{0} & \mathbf{0} & \mathbf{0} & \mathbf{0} & \mathbf{0} & \mathbf{0} & -\frac{1}{\mu} \delta_{i j} & -a \delta_{i j} \\
\mathbf{0} & \mathbf{0} & \mathbf{0} & \mathbf{0} & \mathbf{0} & \mathbf{0} & \delta_{i j} & \mathbf{0} & \mathbf{0} & \mathbf{0} \\
\delta_{i j} & \mathbf{0} & \mathbf{0} & \mathbf{0} & \mathbf{0} & \mathbf{0} & \mathbf{0} & \mathbf{0} & \delta_{i j} & \mathbf{0} \\
\mathbf{0} & \mathbf{0} & \mathbf{0} & \mathbf{0} & \mathbf{0} & \mathbf{0} & \mathbf{0} & \delta_{i j} & \mathbf{0} & \delta_{i j} \\
\mathbf{0} & \mathbf{0} & \mathbf{0} & -\delta_{i j} & \mathbf{0} & \mathbf{0} & \mathbf{0} & \mathbf{0} & \mathbf{0} & \mathbf{0} \\
\mathbf{0} & \mathbf{0} & \mathbf{0} & \mathbf{0} & \mathbf{0} & -\delta_{i j} & \mathbf{0} & \mathbf{0} & \mathbf{0} & \mathbf{0} \\
\mathbf{0} & \mathbf{0} & \frac{1}{\mu} \delta_{i j} & \mathbf{0} & -\delta_{i j} & \mathbf{0} & \mathbf{0} & \mathbf{0} & \mathbf{0} & \mathbf{0} \\
\mathbf{0} & \frac{1}{\mu} \delta_{i j} & a \delta_{i j} & \mathbf{0} & \mathbf{0} & -\delta_{i j} & \mathbf{0} & \mathbf{0} & \mathbf{0} & \mathbf{0}
\end{array}\right)
$$

and substitution of the inverse of $\mathrm{C}$ into Dirac bracket (27) leads to

$$
\begin{aligned}
\{F, G\}_{D B} & =\{F, G\}+a \mu^{2}\left\{F, \Phi_{k}^{(1)}\right\} \delta^{k l}\left\{\Phi_{l}^{(2)}, G\right\}-\mu\left\{F, \Phi_{k}^{(1)}\right\} \delta^{k l}\left\{\Phi_{l}^{(3)}, G\right\}-\left\{F, \Phi_{k}^{(1)}\right\} \delta^{k l}\left\{\Phi_{l}^{\left(\lambda_{2}\right)}, G\right\} \\
& -a \mu^{2}\left\{F, \Phi_{k}^{(2)}, G\right\} \delta^{k l}\left\{\Phi_{l}^{(1)}, G\right\}+\mu\left\{F, \Phi_{k}^{(2)}\right\} \delta^{k l}\left\{\Phi_{l}^{(y)}, G\right\}+a \mu^{2}\left\{F, \Phi_{k}\right\} \delta^{k l}\left\{\Phi_{l}^{(y)}, G\right\}-\left\{F, \Phi_{k}^{(2)}\right\} \delta^{k l}\left\{\phi_{l}, G\right\} \\
& +\mu\left\{F, \Phi_{k}^{(3)}\right\} \delta^{k l}\left\{\Phi_{l}^{(1)}, G\right\}-\mu\left\{F, \Phi_{k}^{(3)}\right\} \delta^{k l}\left\{\Phi_{l}, G\right\}+\left\{F, \Phi_{k}^{\left(\lambda_{1}\right)}\right\} \delta^{k l}\left\{\Phi_{l}^{(x)}, G\right\}+\left\{F, \Phi_{k}^{\left(\lambda_{2}\right)}\right\} \delta^{k l}\left\{\Phi_{l}^{(1)}, G\right\} \\
& +\left\{F, \Phi_{k}^{\left(\lambda_{3}\right)}\right\} \delta^{k l}\left\{\Phi_{l}^{(y)}, G\right\}-\left\{F, \Phi_{k}^{(x)}\right\} \delta^{k l}\left\{\Phi_{l}^{\left(\lambda_{1}\right.}, G\right\}-\mu\left\{F, \Phi_{k}^{(y)}\right\} \delta^{k l}\left\{\Phi_{l}^{(2)}, G\right\}-\left\{F, \Phi_{k}^{(y)}\right\} \delta^{k l}\left\{\Phi_{l}^{\left(\lambda_{3}\right)}, G\right\} \\
& -a \mu^{2}\left\{F, \Phi_{k}\right\} \delta^{k l}\left\{\Phi_{l}^{(2)}, G\right\}+\mu\left\{F, \Phi_{k}\right\} \delta^{k l}\left\{\Phi_{l}^{(3)}, G\right\}+\mu\left\{F, \phi_{k}\right\} \delta^{k l}\left\{\Phi_{l}^{(2)}, G\right\} .
\end{aligned}
$$

Dirac brackets of coordinates are

$$
\begin{aligned}
& \left\{q_{(1)}^{i}, q_{(2)}^{j}\right\}_{D B}=-a \mu^{2} \delta^{i j}, \quad\left\{q_{(1)}^{i}, q_{(3)}^{j}\right\}_{D B}=\mu \delta^{i j}, \quad\left\{q_{(1)}^{i}, \lambda_{2}^{j}\right\}_{D B}=\delta^{i j}, \quad\left\{q_{(1)}^{i}, p_{j}^{(1)}\right\}_{D B}=\delta_{j}^{i} \\
& \left\{q_{(2)}^{i}, y^{j}\right\}_{D B}=-\mu \delta^{i j} \quad\left\{x^{i}, \lambda_{1}^{j}\right\}_{D B}=\delta^{i j}, \quad\left\{y^{i}, \lambda_{3}^{j}\right\}_{D B}=\delta^{i j}, \quad\left\{x^{i}, p_{j}^{(x)}\right\}_{D B}=\delta_{j}^{i}, \quad\left\{y^{i}, p_{j}^{(y)}\right\}_{D B}=\delta_{j}^{i}
\end{aligned}
$$

and all the remaining Dirac brackets of coordinates are zero. Using these Dirac brackets of coordinates and Hamilton function (132) one can easily determine Hamilton equations of motion.

\subsection{Partial reduction I}

We now apply the partial reduction I (c.f. 3.2.1) to the Sarığlu and Tekin Lagrangian (127). So that we rewrite the first order Lagrangian function given in (36) for the case of the Sarığlu and Tekin Lagrangian and arrive at

$$
L_{C_{1}}^{S T}=\frac{1}{2}\left[a\left(\dot{\mathbf{q}}_{(1)}^{2}+\dot{\mathbf{y}}^{2}\right)+\frac{2}{\mu} \dot{\mathbf{y}} \cdot \dot{\mathbf{q}}_{(2)}-m^{2}\left(\mathbf{y}^{2}+\mathbf{q}_{(1)}^{2}\right)\right]+\boldsymbol{\lambda}_{1} \cdot\left(\dot{\mathbf{q}}_{(1)}-\mathbf{q}_{(2)}\right)
$$


using coordinate transformations $\mathbf{x}=\mathbf{q}_{(1)}, \dot{\mathbf{x}}=\dot{\mathbf{q}}_{(1)}=\mathbf{q}_{(2)}, \ddot{\mathbf{x}}=\dot{\mathbf{q}}_{(2)}$ and Lagrange multipliers $\boldsymbol{\lambda}_{1}$. The dual coordinates are $\left(\mathbf{q}_{(1)}, \mathbf{q}_{(2)}, \boldsymbol{\lambda}_{1}, \mathbf{y}\right)$. Using this first order Lagrangian, we compute the conjugate momenta as

$$
\mathbf{p}^{(1)}=a \dot{\mathbf{q}}_{(1)}+\boldsymbol{\lambda}_{1}, \quad \mathbf{p}^{(2)}=\frac{1}{\mu} \dot{\mathbf{y}}, \quad \mathbf{p}^{\lambda_{1}}=\mathbf{0}, \quad \mathbf{p}^{(y)}=a \dot{\mathbf{y}}+\frac{1}{\mu} \dot{\mathbf{q}}_{(2)} .
$$

From these equations we compute the velocities $\dot{\mathbf{y}}, \dot{\mathbf{q}}_{(1)}$ and $\dot{\mathbf{q}}_{(2)}$ in terms of the momenta as follows

$$
\dot{\mathbf{y}}=\mu \mathbf{p}^{(2)}, \quad \dot{\mathbf{q}}_{(1)}=\frac{1}{a}\left(\mathbf{p}^{(1)}-\boldsymbol{\lambda}_{1}\right), \quad \dot{\mathbf{q}}_{(2)}=\mu\left(\mathbf{p}^{(y)}-a \mu \mathbf{p}^{(1)}\right)
$$

whereas from the equation involving the momenta $\mathbf{p}^{\lambda_{1}}$, we have a primary constraint $\boldsymbol{\Phi}^{\left(\lambda_{1}\right)}=\mathbf{p}^{\lambda_{1}}=0$. In this case, the canonical Hamiltonian function is

$$
H_{C_{1}}^{S T}=\mu \mathbf{p}^{(2)} \cdot \mathbf{p}^{(y)}-\frac{a \mu^{2}}{2}\left(\mathbf{p}^{(2)}\right)^{2}+\frac{m^{2}}{2}\left(\mathbf{y}^{2}+\mathbf{q}_{(1)}^{2}\right)+\boldsymbol{\lambda}_{1} \cdot \mathbf{q}_{(2)}+\frac{1}{2 a}\left(\mathbf{p}^{(1)}-\boldsymbol{\lambda}_{1}\right)^{2}
$$

whereas the total Hamiltonian (40) defined to be

$$
H_{T_{1}}^{S T}=H_{C_{1}}^{S T}+\mathbf{u}_{\left(\lambda_{1}\right)} \cdot \boldsymbol{\Phi}^{\left(\lambda_{1}\right)}
$$

by adding the primary constraint with a Lagrange multiplier $\mathbf{u}_{\left(\lambda_{1}\right)}$. Here are the steps of the Dirac-Bergmann constraint algorithm and the computation of the total Hamiltonian function.

Dirac-Bergmann constraint algorithm step 1: Consistency of the primary constraint $\boldsymbol{\Phi}^{\left(\lambda_{1}\right)}$

$$
\dot{\boldsymbol{\Phi}}^{\left(\lambda_{1}\right)}=\frac{1}{a}\left(\mathbf{p}^{(1)}-\boldsymbol{\lambda}_{1}\right)-\mathbf{q}_{(2)}
$$

leads us to a secondary constraint $\boldsymbol{\Phi}=\frac{1}{a}\left(\mathbf{p}^{(1)}-\boldsymbol{\lambda}_{1}\right)-\mathbf{q}_{(2)}$. Note that Eq. (146) will vanish weakly when we use $\dot{\mathbf{q}}_{(1)}=\mathbf{q}_{(2)}$. In this case, it is not possible to determine the Lagrange multiplier $\mathbf{u}_{\left(\lambda_{1}\right)}$, thus equation of motion for $\boldsymbol{\lambda}_{1}$ remains arbitrary. To solve this, we consider $\boldsymbol{\Phi}$ as a secondary constraint. Revised the total Hamiltonian as

$$
H_{T_{2}}^{S T}=H_{C_{1}}^{S T}+\mathbf{u}_{\left(\lambda_{1}\right)} \cdot \boldsymbol{\Phi}^{\left(\lambda_{1}\right)}+\mathbf{u} \cdot \boldsymbol{\Phi}
$$

by adding secondary constraint with Lagrange multiplier $\mathbf{u}$.

Dirac-Bergmann constraint algorithm step 2: The consistency of the secondary constraint $\Phi$ can be checked through

$$
\dot{\mathbf{\Phi}}=\left\{\boldsymbol{\Phi}, H_{T_{2}}^{S T}\right\} \approx-\frac{m^{2}}{a} \mathbf{q}_{(1)}+a \mu^{2} \mathbf{p}^{(2)}-\mu \mathbf{p}^{(y)}-\frac{1}{a} \mathbf{u}_{\left(\lambda_{1}\right)}
$$

which leads us to determine the Lagrange multiplier $\mathbf{u}_{\left(\lambda_{1}\right)}$ as $\mathbf{u}_{\left(\lambda_{1}\right)} \approx-m^{2} \mathbf{q}_{(1)}+a^{2} \mu^{2} \mathbf{p}^{(2)}-a \mu \mathbf{p}^{(y)}$. On the other hand, the consistency of $\boldsymbol{\Phi}^{\left(\lambda_{1}\right)}$

$$
\dot{\boldsymbol{\Phi}}^{\left(\lambda_{1}\right)}=\left\{\boldsymbol{\Phi}^{\left(\lambda_{1}\right)}, H_{T_{2}}^{S T}\right\} \approx \boldsymbol{\Phi}+\frac{1}{a} \mathbf{u}
$$

leads to us to determine $\mathbf{u} \approx-a \boldsymbol{\Phi}$.

The total Hamiltonian function and the equations of motion: Substitutions of the Lagrange multipliers $\mathbf{u}_{\left(\lambda_{1}\right)}$ and $\mathbf{u}$ into (147) give the total Hamiltonian function

$$
\begin{aligned}
H_{T_{2}}^{S T} & =\mu \mathbf{p}^{(2)} \cdot\left(\mathbf{p}^{(y)}-\frac{a \mu}{2} \mathbf{p}^{(2)}\right)+\frac{m^{2}}{2}\left(\mathbf{y}^{2}+\mathbf{q}_{(1)}^{2}\right)+\boldsymbol{\lambda}_{1} \cdot \mathbf{q}_{(2)}+\frac{1}{2 a}\left(\boldsymbol{p}^{(1)}-\boldsymbol{\lambda}_{1}\right)^{2} \\
& -\left(m^{2} \mathbf{q}_{(1)}-a^{2} \mu^{2} \mathbf{p}^{(2)}+a \mu \mathbf{p}^{(y)}\right) \cdot \mathbf{p}^{\lambda_{1}}-\frac{1}{a}\left(\mathbf{p}^{(1)}-\boldsymbol{\lambda}_{1}-\mathbf{q}_{(2)}\right)^{2} .
\end{aligned}
$$


The Hamilton's equations of motion using are

$$
\begin{aligned}
\dot{\mathbf{q}}_{(1)} & \approx \frac{1}{a}\left(\mathbf{p}^{(1)}-\boldsymbol{\lambda}_{1}\right), \quad \dot{\mathbf{q}}_{(2)} \approx \mu\left(\mathbf{p}^{(y)}-a \mu^{2} \mathbf{p}^{(2)}\right), \quad \dot{\mathbf{y}} \approx \mu \mathbf{p}^{(2)} \\
\dot{\boldsymbol{\lambda}}_{1} & \approx-m^{2} \mathbf{q}_{(1)}+a^{2} \mu^{2} \mathbf{p}^{(2)}-a \mu \mathbf{p}^{(y)}, \quad \dot{\boldsymbol{p}}^{(1)} \approx-m^{2} \mathbf{q}_{(1)} \\
\dot{\mathbf{p}}^{(2)} & \approx-\boldsymbol{\lambda}_{1}, \quad \dot{\mathbf{p}}^{\lambda_{1}} \approx \frac{1}{a}\left(\mathbf{p}^{(1)}-\boldsymbol{\lambda}_{1}\right)-\mathbf{q}_{(2)}, \quad \dot{\mathbf{p}}^{(y)} \approx-m^{2} \mathbf{y} .
\end{aligned}
$$

The Dirac bracket: The constraints

$$
\boldsymbol{\Phi}=\frac{1}{a}\left(\mathbf{p}^{(1)}-\boldsymbol{\lambda}_{1}\right)-\mathbf{q}_{(2)}, \quad \boldsymbol{\Phi}^{\left(\lambda_{1}\right)}=\mathbf{p}^{\lambda_{1}}
$$

for the first order Lagrangian $L_{C_{(1)}}^{S T}$ are of the second class since their Poisson bracket $\left\{\Phi^{i}, \Phi_{j}^{\left(\lambda_{1}\right)}\right\}=\frac{1}{a} \delta_{j}^{i}$ is nonzero. Recall the definition of the Dirac bracket presented in (27). In particular, for the constraints (152), we arrive at

$$
\{F, G\}_{D B}=\{F, G\}+a\left\{F, \Phi^{k}\right\} \delta_{k}^{n}\left\{\Phi_{n}^{\left(\lambda_{1}\right)}, G\right\}-a\left\{F, \Phi_{k}^{\left(\lambda_{1}\right)}\right\} \delta_{n}^{k}\left\{\Phi^{n}, G\right\}
$$

after the substitution of the inverse matrix of

$$
C=\left[\begin{array}{cc}
\left\{\Phi^{k}, \Phi^{n}\right\} & \left\{\Phi^{k}, \Phi_{n}^{\left(\lambda_{1}\right)}\right\} \\
\left\{\Phi_{n}^{\left(\lambda_{1}\right)}, \Phi^{k}\right\} & \left\{\Phi_{n}^{\left(\lambda_{1}\right)}, \Phi_{k}^{\left(\lambda_{1}\right)}\right\}
\end{array}\right]=\frac{1}{a}\left[\begin{array}{cc}
0_{3 \times 3} & \delta_{n}^{k} \\
-\delta_{n}^{k} & 0_{3 \times 3}
\end{array}\right] .
$$

We compute the Dirac brackets of the coordinates as follows

$$
\begin{aligned}
& \left\{q_{(1)}^{i}, \lambda_{1}^{j}\right\}_{D B}=\delta^{i j}, \quad\left\{q_{(1)}^{i}, p_{j}^{(1)}\right\}_{D B}=\delta_{j}^{i}, \quad\left\{q_{(2)}^{i}, p_{j}^{(2)}\right\}_{D B}=\delta_{j}^{i} \\
& \left\{\lambda_{1}^{i}, p_{j}^{(2)}\right\}_{D B}=-a \delta_{j}^{i}, \quad\left\{y^{i}, p_{j}^{(y)}\right\}_{D B}=\delta_{j}^{i}
\end{aligned}
$$

and all rest is zero. Using these Dirac brackets of coordinates and Hamiltonian function (144) we can recover the equations of motion (151) after a direct computation.

\subsection{Partial reduction II}

In this subsection, we are applying the method of partial reduction II, which is presented in the subsection (3.2.2), to the Sarığlu-Tekin Lagrangian (127). Accordingly, we compute

$$
L_{C_{2}}^{S T}=\frac{1}{2}\left[a\left(\mathbf{q}_{(2)}^{2}+\dot{\mathbf{y}}^{2}\right)+\frac{2}{\mu} \dot{\mathbf{y}} \cdot \dot{\mathbf{q}}_{(2)}-m^{2}\left(\mathbf{y}^{2}+\mathbf{q}_{(1)}^{2}\right)\right]+\boldsymbol{\lambda}_{2} \cdot\left(\dot{\mathbf{q}}_{(1)}-\mathbf{q}_{(2)}\right)
$$

using the coordinate transformations $\mathbf{x}=\mathbf{q}_{(1)}, \dot{\mathbf{x}}=\dot{\mathbf{q}}_{(1)}=\mathbf{q}_{(2)}$, and $\ddot{\mathbf{x}}=\dot{\mathbf{q}}_{(2)}$. Here, $\boldsymbol{\lambda}_{2}$ stands for a set of Lagrange multipliers. The fiber derivatives of $L_{C_{2}}^{S T}$ establish the relationship between the velocities and the momenta as follows

$$
\mathbf{p}^{(1)}=\boldsymbol{\lambda}_{2}, \quad \mathbf{p}^{(2)}=\frac{1}{\mu} \dot{\mathbf{y}}, \quad \mathbf{p}^{\lambda_{2}}=0, \quad \mathbf{p}^{(y)}=a \dot{\mathbf{y}}+\frac{1}{\mu} \dot{\mathbf{q}}_{(2)} .
$$

From the conjugate momenta (157), it is possible to solve $\dot{\mathbf{y}}$ and $\dot{\mathbf{q}}_{(2)}$ as functions of coordinates and momenta

$$
\dot{\mathbf{y}}=\mu \mathbf{p}^{(2)}, \quad \dot{\mathbf{q}}_{(2)}=\mu\left(\mathbf{p}^{(y)}-a \mu \mathbf{p}^{(2)}\right),
$$

but, the others lead to primary constraints

$$
\boldsymbol{\Phi}^{(1)}=\mathbf{p}^{(1)}-\boldsymbol{\lambda}_{2}, \quad \boldsymbol{\Phi}^{\left(\lambda_{2}\right)}=\mathbf{p}^{\lambda_{2}} .
$$

In this case, the canonical Hamiltonian function becomes

$$
H_{C_{2}}^{S T}=\left(\mu \mathbf{p}^{(2)} \cdot \mathbf{p}^{(y)}-\frac{a \mu^{2}}{2}\left(\mathbf{p}^{(2)}\right)^{2}\right)-\frac{a}{2} \mathbf{q}_{(2)}+\boldsymbol{p}^{(1)} \cdot \mathbf{q}_{(2)}+\frac{m^{2}}{2}\left(\mathbf{y}^{2}+\mathbf{q}_{(1)}^{2}\right)
$$


whereas the total Hamiltonian function is defined to be

$$
H_{T}^{S T}=H_{C_{2}}^{S T}+\mathbf{u}_{(1)} \cdot \boldsymbol{\Phi}^{(1)}+\mathbf{u}_{\left(\lambda_{2}\right)} \cdot \boldsymbol{\Phi}^{\left(\lambda_{2}\right)} .
$$

Here, $\mathbf{u}_{(1)}$ and $\mathbf{u}_{\left(\lambda_{2}\right)}$ Lagrange multipliers. Here are the steps of the Dirac-Bergmann constraint algorithm.

Dirac-Bergmann constraint algorithm step 1: The consistency checks of the primary constraints

$$
\dot{\boldsymbol{\Phi}}^{(1)}=\left\{\boldsymbol{\Phi}^{(1)}, H_{T}^{S T}\right\} \approx-m^{2} \mathbf{q}_{(1)}-\mathbf{u}_{\left(\lambda_{2}\right)}, \quad \dot{\boldsymbol{\Phi}}^{\left(\lambda_{2}\right)}=\left\{\boldsymbol{\Phi}^{\left(\lambda_{2}\right)}, H_{T}^{S T}\right\} \approx \mathbf{u}_{(1)}
$$

allow us to determine the multipliers as $\mathbf{u}_{(1)} \approx 0$ and $\mathbf{u}_{\left(\lambda_{2}\right)} \approx-m^{2} \mathbf{q}_{(0)}$, respectively.

The total Hamiltonian function and the equations of motion: Substitutions of the Lagrange multipliers $\mathbf{u}_{(1)}$ and $\mathbf{u}_{\left(\lambda_{2}\right)}$ into (161) determine the total Hamiltonian $H_{T}^{S T}$ in the following explicit form

$$
H_{T}^{S T}=\mu \mathbf{p}^{(2)} \cdot\left(\mathbf{p}^{(y)}-\frac{a \mu}{2} \mathbf{p}^{(2)}\right)-\frac{a}{2} \mathbf{q}_{(2)}^{2}+\mathbf{p}^{(1)} \cdot \mathbf{q}_{(2)}+\frac{m^{2}}{2}\left(\mathbf{y}^{2}+\mathbf{q}_{(1)}^{2}\right)-m^{2} \mathbf{q}_{(1)} \cdot \mathbf{p}^{\lambda_{2}} .
$$

The Hamilton's equations are

$$
\begin{aligned}
& \dot{\mathbf{q}}_{(1)} \approx \mathbf{q}_{(2)}, \quad \dot{\mathbf{q}}_{(2)} \approx \mu \mathbf{p}^{(y)}-a \mu^{2} \mathbf{p}^{(2)}, \quad \dot{\mathbf{y}} \approx \mu \mathbf{p}^{(2)}, \quad \dot{\lambda}_{2} \approx-m^{2} \mathbf{q}_{(1)} \\
& \dot{\mathbf{p}}^{(2)} \approx a \mathbf{q}_{(2)}-\mathbf{p}^{(1)}, \quad \dot{\mathbf{p}}^{\lambda_{2}} \approx 0, \quad \dot{\mathbf{p}}^{(1)} \approx-m^{2} \mathbf{q}_{(1)}, \quad \dot{\mathbf{p}}^{(y)} \approx-m^{2} \mathbf{y} .
\end{aligned}
$$

The Dirac bracket: Now we are going to arrive at the Hamilton's equations by defining the Dirac bracket

$$
\{F, G\}_{D B}=\{F, G\}-\left\{F, \Phi_{k}^{(1)}\right\} \delta^{k n}\left\{\Phi_{n}^{\left(\lambda_{2}\right)}, G\right\}+\left\{F, \Phi_{k}^{\left(\lambda_{2}\right)}\right\} \delta^{k n}\left\{\Phi_{n}^{(1)}, G\right\},
$$

where we substitute the inverse of matrix

$$
C=\left(\begin{array}{cc}
\left\{\Phi_{k}^{(1)}, \Phi_{n}^{(1)}\right\} & \left\{\Phi_{k}^{(1)}, \Phi_{n}^{\left(\lambda_{2}\right)}\right\} \\
\left\{\Phi_{k}^{\left(\lambda_{2}\right)}, \Phi_{n}^{(1)}\right\} & \left\{\Phi_{k}^{\left(\lambda_{2}\right)}, \Phi_{\lambda}^{n}\right\}
\end{array}\right)=\left(\begin{array}{cc}
0_{3 \times 3} & -\delta_{k n} \\
\delta_{k n} & 0_{3 \times 3}
\end{array}\right) .
$$

For the constraint space defined by (159), the Dirac brackets of the coordinates are

$$
\left\{q_{(1)}^{i}, \lambda_{2}^{j}\right\}_{D B}=\delta^{i j}, \quad\left\{q_{(1)}^{i}, p_{j}^{(1)}\right\}_{D B}=\delta_{j}^{i}, \quad\left\{q_{(2)}^{i}, p_{j}^{(2)}\right\}_{D B}=\delta_{j}^{i}, \quad\left\{y^{i}, p_{j}^{(y)}\right\}_{D B}=\delta_{j}^{i}
$$

and the rest is zero.

Remark 4 (Constraints for partial reductions I and II) In the following table we list the constraints derived from the Lagrangian functions $L_{C_{(1)}}^{S T}$ in Eq. 141) and $L_{C_{(2)}}^{S T}$ in Eq.(156). Observe that, to arrive at an identification between these two constraint systems, it is enough to take $\boldsymbol{\lambda}_{1}=\boldsymbol{\lambda}_{2}-a \mathbf{q}_{2}$.

\begin{tabular}{|c|c|c|}
\hline & Partial Reduction I & Partial Reduction II \\
\hline Reduction & $\mathbf{x}=\mathbf{q}_{(1)}, \dot{\mathbf{x}}=\mathbf{q}_{(2)}, \ddot{\mathbf{x}}=\dot{\mathbf{q}}_{(2)}$ & $\mathbf{x}=\mathbf{q}_{(1)}, \dot{\mathbf{x}}=\dot{\mathbf{q}}_{(1)}, \ddot{\mathbf{x}}=\dot{\mathbf{q}}_{(2)}$ \\
\hline Coordinates & $\mathbf{q}_{(1)}, \mathbf{q}_{(2)}, \mathbf{y}, \boldsymbol{\lambda}_{1}, \mathbf{p}^{(1)}, \mathbf{p}^{(2)}, \mathbf{p}^{(y)}, \mathbf{p}^{\left(\lambda_{1}\right)}$ & $\mathbf{q}_{(1)}, \mathbf{q}_{(2)}, \mathbf{y}, \boldsymbol{\lambda}_{2}, \mathbf{p}^{(1)}, \mathbf{p}^{(2)}, \mathbf{p}^{(y)}, \mathbf{p}^{\left(\lambda_{2}\right)}$ \\
\hline \multirow{2}{*}{ Primary Constraints } & $\mathbf{\Phi}^{\left(\lambda_{1}\right)}=\mathbf{p}^{\left(\lambda_{1}\right)}$ & $\boldsymbol{\Phi}^{(1)}=\mathbf{p}^{(1)}-\lambda_{2}$ \\
& & $\mathbf{\Phi}^{\left(\lambda_{2}\right)}=\mathbf{p}^{\lambda_{2}}$ \\
\hline Secondary Constraints & $\mathbf{\Phi}=\frac{1}{a}\left(\mathbf{p}^{(1)}-\lambda_{1}\right)-\mathbf{q}_{(2)}$ & \\
\hline
\end{tabular}




\subsection{Deriglazov's trick / Schmidt's method}

Finally, we are now applying the method in the subsection (3.3.2) to the Sarığlu-Tekin Lagrangian (127). Accordingly, following the definition in (53), we introduce the first order Lagrangian

$$
L_{A}^{S T}=\frac{1}{2}\left[a\left(\dot{\mathbf{x}}^{2}+\dot{\mathbf{y}}^{2}\right)+\frac{2}{\mu} \dot{\mathbf{y}} \cdot \mathbf{q}_{(3)}-m^{2}\left(\mathbf{x}^{2}+\mathbf{y}^{2}\right)\right]-\dot{\gamma} \cdot \dot{\mathbf{x}}-\gamma \cdot \mathbf{q}_{(3)}
$$

depending on the base variables $\left(\mathbf{x}, \mathbf{y}, \mathbf{q}_{(3)}, \gamma\right)$ and the velocity variables $\left(\dot{\mathbf{x}}, \dot{\mathbf{y}}, \dot{\mathbf{q}}_{(3)}, \dot{\gamma}\right)$. Introduce the dual coordinates as $\left(\mathbf{p}^{(x)}, \mathbf{p}^{(y)}, \mathbf{p}^{(3)}, \mathbf{p}^{(\gamma)}\right)$. The Legendre transformation reads the following equations

$$
\mathbf{p}^{(x)}=a \dot{\mathbf{x}}-\dot{\gamma}, \mathbf{p}^{(y)}=a \dot{\mathbf{y}}+\frac{1}{\mu} \mathbf{q}_{(3)}, \mathbf{p}^{(3)}=\mathbf{0}, \mathbf{p}^{(\gamma)}=-\dot{\mathbf{x}} .
$$

We can solve the velocities $\dot{\mathbf{x}}, \dot{\mathbf{y}}, \dot{\gamma}$ in terms of the momenta and there remains a set of primary constraint $\varphi=$ $\mathbf{p}^{(3)} \approx 0$. The total Hamiltonian function is defined to be

$$
H_{T}=-\mathbf{p}^{(x)} \cdot \mathbf{p}^{(\gamma)}+\frac{1}{2}\left[-a\left(\mathbf{p}^{(\gamma)}\right)^{2}+\frac{1}{a}\left(\mathbf{p}^{(y)}-\frac{1}{\mu} \mathbf{q}_{(3)}\right)^{2}\right]+\frac{m^{2}}{2}\left(\mathbf{x}^{2}+\mathbf{y}^{2}\right)+\gamma \cdot \mathbf{q}_{(3)}+\boldsymbol{w} \cdot \mathbf{p}^{(3)}
$$

where $\boldsymbol{w}$ is a set of Lagrange multipliers. Here are the steps of the Dirac-Bergmann constraint algorithm and the computation of the total Hamiltonian function.

Dirac-Bergmann constraint algorithm step 1: Consistency condition of primary constraint $\varphi$

$$
\dot{\varphi}=\left\{\boldsymbol{\varphi}, H_{T}\right\} \approx \frac{1}{a}\left(\mathbf{p}^{(y)}-\frac{1}{\mu} \mathbf{q}_{(3)}\right)-\gamma
$$

gives secondary constraint $\boldsymbol{\Phi}=\frac{1}{a}\left(\mathbf{p}^{(y)}-\frac{1}{\mu} \mathbf{q}_{(3)}\right)-\gamma$. We are revising the total Hamiltonian as $H_{T 1}=H_{T}+\overline{\boldsymbol{w}} \cdot \boldsymbol{\Phi}$.

Dirac-Bergmann constraint algorithm step 2: Conservation of the secondary constraint $\boldsymbol{\Phi}$

$$
\dot{\mathbf{\Phi}}=\left\{\boldsymbol{\Phi}, H_{T 1}\right\} \approx-\frac{1}{a} m^{2} \mathbf{y}-\frac{1}{a \mu} \boldsymbol{w}+\mathbf{p}^{(x)}-a \mathbf{p}^{(\gamma)}
$$

determine $\boldsymbol{w}$ and conservation of $\boldsymbol{\varphi}$

$$
\dot{\boldsymbol{\varphi}}=\left\{\boldsymbol{\varphi}, H_{T 1}\right\} \approx \mathbf{\Phi}+\frac{1}{a \mu} \overline{\boldsymbol{w}}
$$

gives $\overline{\boldsymbol{w}} \approx 0$.

The total Hamiltonian function and the equations of motion: Thus substitutions of $\boldsymbol{w}$ and $\overline{\boldsymbol{w}}$ into total Hamilton $H_{T 1}$ give

$$
\begin{aligned}
H_{T 1} & =-\mathbf{p}^{(x)} \cdot \mathbf{p}^{(\gamma)}+\frac{1}{2}\left(-a\left(\mathbf{p}^{(\gamma)}\right)^{2}+\frac{1}{a}\left(\mathbf{p}^{(y)}-\frac{1}{\mu} \mathbf{q}_{(3)}\right)^{2}\right)+\frac{m^{2}}{2}\left(\mathbf{x}^{2}+\mathbf{y}^{2}\right)+\gamma \cdot \mathbf{q}_{(3)} \\
& +\mathbf{p}^{(3)} \cdot\left(-m^{2} \mu \mathbf{y}+a \mu^{2}\left(\mathbf{p}^{(x)}+a \mathbf{p}^{(\gamma)}\right)\right)-a \mu\left(\frac{1}{a}\left(\mathbf{p}^{(y)}-\frac{1}{\mu} \mathbf{q}_{(3)}\right)-\gamma\right)^{2} .
\end{aligned}
$$

The Hamilton's equations of motion generated by this total Hamiltonian function are

$$
\begin{aligned}
\dot{\mathbf{x}} & \approx-\mathbf{p}^{(\gamma)}, \dot{\mathbf{y}} \approx \frac{1}{a}\left(\mathbf{p}^{(y)}-\frac{1}{\mu} \mathbf{q}_{(3)}\right), \dot{\gamma} \approx-\mathbf{p}^{x}-a \mathbf{p}^{(\gamma)} \\
\dot{\mathbf{q}}_{(3)} & \approx-m^{2} \mu \mathbf{y}+a \mu^{2}\left(\mathbf{p}^{(x)}+a \mathbf{p}^{(\gamma)}\right), \dot{\mathbf{p}}^{(\gamma)} \approx-\mathbf{q}_{(3)}, \dot{\mathbf{p}}^{(3)} \approx 0, \\
\dot{\mathbf{p}}^{(y)} & \approx-m^{2} \mathbf{y}, \dot{\mathbf{p}}^{(x)} \approx-m^{2} \mathbf{x}
\end{aligned}
$$

From these equations, first two lines of equations satisfied identically and the last one give Euler-Lagrange equations (128) using the definitions of momenta. Here is the list of constraints. 


\begin{tabular}{|c|c|}
\hline & Deriglazov's trick / Schmidt's method \\
\hline Reduction & $\ddot{\mathbf{x}}=\mathbf{q}_{(3)}, \gamma=\frac{\partial L}{\partial \mathbf{q}_{(3)}}=\frac{1}{\mu} \dot{\mathbf{y}}-\gamma$, \\
\hline Coordinates & $\mathbf{x}, \mathbf{y}, \mathbf{q}_{(3)}, \gamma, \mathbf{p}^{(x)}, \mathbf{p}^{(y)}, \mathbf{p}^{(3)}, \mathbf{p}^{(\gamma)}$ \\
\hline Primary Constraints & $\varphi=\mathbf{p}^{(3)}$ \\
\hline Secondary constraints & $\mathbf{\Phi}=\frac{1}{a}\left(\mathbf{p}^{(y)}-\frac{1}{\mu} \mathbf{q}_{(3)}\right)-\gamma$ \\
\hline
\end{tabular}

The Dirac bracket: All the constraints

$$
\boldsymbol{\varphi}=\mathbf{p}^{(3)}, \quad \boldsymbol{\Phi}=\frac{1}{a \mu}\left(\mathbf{p}^{(y)}-\frac{1}{\mu} \mathbf{q}_{(3)}\right)-\gamma
$$

are second class since the Poisson brackets $\left\{\boldsymbol{\varphi}_{i}, \mathbf{\Phi}_{j}\right\}=\frac{1}{a \mu^{2}} \delta_{i j}$ are nonzero. In this case Dirac bracket (27) becomes

$$
\{F, G\}_{D B}=\{F, G\}+\{F, \boldsymbol{\varphi}\} a \mu^{2}\{\boldsymbol{\Phi}, G\}-\{F, \boldsymbol{\Phi}\} a \mu^{2}\{\boldsymbol{\varphi}, G\} .
$$

The Dirac brackets of coordinates are

$$
\begin{aligned}
& \left\{x^{i}, p_{j}^{(x)}\right\}_{D B}=\left\{y^{i}, p_{j}^{(y)}\right\}_{D B}=\left\{\gamma^{i}, p_{j}^{(\gamma)}\right\}_{D B}=\delta_{j}^{i} \\
& \left\{y^{i}, q_{(3)}^{j}\right\}_{D B}=\mu \delta^{i j}, \quad\left\{q_{(3)}^{i}, p_{j}^{(\gamma)}\right\}_{D B}=-a \mu^{2} \delta_{j}^{i}
\end{aligned}
$$

and the rest are identically zero. From these Dirac brackets of coordinates and Hamiltonian function (170) one obtains the Hamilton's equations (175) after a straight forward calculation.

\section{Conclusions}

We have presented several different ways enabling to reduce a second order Lagrangian function to a first order one in Section 3. The geometric construction presented in sections 2 and 3 clarifies the relationships between these methods. We have particularly interested in two second order degenerate Lagrangian theories available in the theory of topologically massive gravity, namely the Clèment Lagrangian (1), and the Sarığlu-Tekin Lagrangian (2). For each of them, using the methods presented in Section 3 we have introduced different first order Lagrangian functions so that different Hamiltonian realizations in Sections (4) and (5), respectively. As a result, we have completed the Hamiltonian analysis of the Clèment, and the Sarığlu-Tekin Lagrangians.

Both the Clément and the Sarığlu-Tekin Lagrangians have rotational symmetry. In [30], the higher dimensional version of Lagrangian reduction theory [11, 50] has been presented. Motivated by this, we are planning to exhibit formal reductions of these theories under rotational symmetry in a future study.

\section{Acknowledgment}

We are grateful Prof. Alexei Deriglazov for his comments attracting our attention to [23].

\section{References}

[1] Abraham, R. and Marsden, J. E. (1978). Foundations of mechanics. Reading, Massachusetts: Benjamin/Cummings Publishing Company.

[2] Andrzejewski, K., Gonera, J., Machalski, P., \& Maślanka, P. (2010). Modified Hamiltonian formalism for higher-derivative theories. Physical Review D, 82(4), 045008.

[3] Andrzejewski, K., Gonera, J., and Maslanka, P. (2007). A note on the Hamiltonian formalism for higherderivative theories. arxiv preprint arXiv:0710.2976. 
[4] Barbero-Liñán, M., Echeverría-Enríquez, A., De Diego, D. M., Munoz-Lecanda, M. C., \& Román-Roy, N. (2007). Skinner-Rusk unified formalism for optimal control systems and applications. Journal of Physics A: Mathematical and Theoretical, 40(40), 12071.

[5] C. Batlee, J. Gomis, J.M. Pons and N. Roman-Roy, Lagrangian and Hamiltonian constraints for second-order singular Lagrangians, J. Phys. A: Math. Gen. 21 (1988) 2693-2703.

[6] P. G. Bergmann, Helv. Phys. Acta, Suppl. 4, 79 (1956).

[7] K. Bolonek and P. Kosiński, Hamiltonian structures for Pais-Uhlenbeck oscillator, Acta Phys. Polonica 36 (2005) 2115-2131.

[8] Çă̆atay Uçgun F., Esen O. and Gümral H., Reductions of topologically massive gravity I: Hamiltonian analysis of second order degenerate Lagrangians, Journal of Mathematical Physics 59, 013510 (2018),https://doi.org/10.1063/1.5021948.

[9] Campos, C. M., de León, M., De Diego, D. M., and Vankerschaver, J. (2009). Unambiguous formalism for higher order Lagrangian field theories. Journal of Physics A: Mathematical and Theoretical, 42(47), 475207.

[10] Cartan, La géométrie de l'intégrale $\int F(x, y, y, y, y) d x$, Quevres Complétes Part III. Vol 1. Gauthiers-Villant, Paris (1955) 1341-1368.

[11] Cendra, H., Marsden, J. E., and Ratiu, T. S. (2001). Lagrangian reduction by stages (No. 722). American Mathematical Soc.

[12] Chen, T. J., Fasiello, M., Lim, E. A., and Tolley, A. J. (2013). Higher derivative theories with constraints: Exorcising Ostrogradski's Ghost. Journal of Cosmology and Astroparticle Physics, 2013(02), 042.

[13] G. Clément, Classical Quantum Gravity 9, 2615 (1992).

[14] G. Clément, Phys. Rev. D 49, 5131 (1994).

[15] G. Clément, Classical and Quantum Gravity 11, 115 (1994)

[16] Crampin, M., Sarlet, W., and Cantrijn, F. (1986, May). Higher-order differential equations and higher-order Lagrangian mechanics. In Mathematical Proceedings of the Cambridge Philosophical Society (Vol. 99, No. 03, pp. 565-587). Cambridge University Press.

[17] Colombo, L., and de Diego D.M., (2011). On the geometry of higher-order variational problems on Lie groups. Journal Geometric Mechanics, Vol. 6 (4), 451-478, 2014.

[18] Colombo, L., de León, M., Prieto-Martínez, P. D., \& Román-Roy, N. (2014). Unified formalism for the generalized kth-order Hamilton-Jacobi problem. International Journal of Geometric Methods in Modern Physics, 11(09), 1460037.

[19] Colombo, L., \& Prieto-Martínez, P. D. (2016). Regularity properties of fiber derivatives associated with higherorder mechanical systems. arxiv preprint arXiv:1602.02717.

[20] Cruz, M., Molgado, A., \& Rojas, E. (2013). Hamiltonian dynamics of linear affine in acceleration theories. arxiv preprint arXiv:1310.5750

[21] Cruz, M., Gómez-Cortés, R., Molgado, A., \& Rojas, E. (2016). Hamiltonian analysis for linearly accelerationdependent Lagrangians. Journal of Mathematical Physics, 57(6), 062903.

[22] Dunin-Barkowski, P. I., \& Sleptsov, A. V. (2009). Geometric Hamiltonian formalism for reparameterizationinvariant theories with higher derivatives. Theoretical and Mathematical Physics, 158(1), 61-81.

[23] A. Deriglazov, Classical Mechanics: Hamiltonian and Lagrangian Formalism, (Springer, 2010)(DOI 10.1007/978-3-642-14037-2)

[24] G. H. Derrick, Classical mechanics with respect to an observer's past light cone, J. Math. Phys. 28 (1987) 64-70. 
[25] Deser, S., Jackiw, R., \& Templeton, S. (1982). Topologically massive gauge theories, Annals of Physics, 140(2), $372-411$.

[26] Deser, S., Jackiw, R., \& Templeton, S. (1982). Three-dimensional massive gauge theories. Physical Review Letters, 48(15), 975-978.

[27] Dirac, P.A.M. (1964). Lectures on Quantum Mechanics, Belfer Graduate School of Science, Monograph Series, yeshiva University, New york.

[28] Dirac, P. A. (1958, August). Generalized hamiltonian dynamics. In Proceedings of the Royal Society of London A: Mathematical, Physical and Engineering Sciences (Vol. 246, No. 1246, pp. 326-332). The Royal Society.

[29] Esen, O., Guha, P. (2018) On the geometry of the Schmidt-Legendre transformation. Journal of Geometric Mechanics, 2018, 10 (3) : 251-291

[30] Gay-Balmaz, F., Holm, D. D., \& Ratiu, T. S. (2011). Higher order Lagrange-Poincaré and Hamilton-Poincaré reductions. Bulletin of the Brazilian Mathematical Society, 42(4), 579-606.

[31] V. Gerdt, A. Khvedelidze, Yu Palii, in Proceedings of GIFT (Cockcroft Institute, Daresbury, UK, 2006), p. 135.

[32] M. J. Gotay and J. M. Nester, In Annales de l'IHP Phys. Théo. 30, 129 (1979)

[33] Gotay, M. J., and Nester, J. M. (1980). Generalized constraint algorithm and special presymplectic manifolds. In Geometric Methods in Mathematical Physics (pp. 78-104). Springer Berlin Heidelberg.

[34] Gotay, M. J., and Nester, J. M. (1984). Apartheid in the Dirac theory of constraints. Journal of Physics A: Mathematical and General, 17(15), 3063.

[35] Gotay, M. J., Nester, J. M., and Hinds, G. (1978). Presymplectic manifolds and the Dirac Bergmann theory of constraints. Journal of Mathematical Physics, 19(11), 2388-2399.

[36] J. Govaerts, and M. S. Rashid, e-print arXiv:hep-th/9403009 (1994).

[37] Grabowski, J., \& Rotkiewicz, M. (2009). Higher vector bundles and multi-graded symplectic manifolds. Journal of Geometry and Physics, 59(9), 1285-1305.

[38] X. Gracia, J. M. Pons, and N. Román-Roy, (1991). J. Math. Phys. 32, 2744 (1991).

[39] M. Horák and I. KolářHorsk, Czeck. Math. J. 33, 467-475, (1983).

[40] Kasper, U. (1997). Finding the Hamiltonian for Cosmological Models in Fourth-Order Gravity Theories Without Resorting to the Ostrogradski or Dirac Formalism. General Relativity and Gravitation, 29(2), 221-233.

[41] J. Keller and S. Rodriguez-Romo, Multivectorial generalization of the Cartan map, J. Math. Phys. 32 (1991) 1591-1598.

[42] S. S. Khalil and M. S. Rashid, Hamiltonian description of higher order Lagrangians, Int. J. of Mod. Phys. 11 (1996) 4551

[43] Kondo, K. (1991). Epistemological foundations of quasi-microscopic phenomena from the standpoint of Finsler's and Kawaguchi's higher order geometry, Post RAAG Reports Nos. 241,242, 243,

[44] Krupková, O. (1994). A geometric setting for higher-order Dirac-Bergmann theory of constraints. Journal of Mathematical Physics, 35(12), 6557-6576.

[45] de León, M., Marín-Solano, J., Marrero, J. C., Muñoz-Lecanda, M. C., and Román-Roy, N. (2002). Singular Lagrangian Systems on Jet Bundles. Fortschritte der Physik, 50, 105-169.

[46] de León, M., Pitanga, P., and Rodrigues, P. R. (1994). Symplectic reduction of higher order Lagrangian systems with symmetry. Journal of Mathematical Physics, 35(12), 6546-6556. 
[47] de León, M., and Rodrigues, P. R. (2011). Generalized Classical Mechanics and Field Theory: a geometrical approach of Lagrangian and Hamiltonian formalisms involving higher order derivatives (Vol. 112). Elsevier.

[48] Lukierski J., Stichel P.C., Zakrzewski W.J., Galilean-invariant (2+1)-dimensional models with a Chern-Simonslike term and $\mathrm{D}=2$ noncommutative geometry, Ann. Physics 260 (1997), 224-249,

[49] Mannheim, P. D., and Davidson, A. (2005). Dirac quantization of the Pais-Uhlenbeck fourth order oscillator. Physical Review A, 71(4), 042110.

[50] J. E. Marsden and T. Ratiu, Introduction to Mechanics and Symmetry: a Basic Exposition of Classical Mechanical Systems, (Springer Science and Business Media, 1998).

[51] Masterov, I. (2016). An alternative Hamiltonian formulation for the Pais-Uhlenbeck oscillator, Nuclear Physics B, 902, 95-114.

[52] Masterov, I. (2016). The odd-order Pais-Uhlenbeck oscillator. Nuclear Physics B, 907, 495-508.

[53] Mostafazadeh, A. (2010). A Hamiltonian formulation of the Pais-Uhlenbeck oscillator that yields a stable and unitary quantum system, Physics Letters A, 375(2), 93-98.

[54] Nakamura, T., and Hamamoto, S. (1996). Higher derivatives and canonical formalisms. Progress of Theoretical Physics, 95(3), 469-484.

[55] Nesterenko, V. V. (1989). Singular Lagrangians with higher derivatives. Journal of Physics A: Mathematical and General, 22(10), 1673.

[56] M. Ostrogradsky, Mem. Ac. St. Petersbourg VI 4, 385 (1850).

[57] Pais, A., and Uhlenbeck, G. E. (1950). On field theories with non-localized action. Physical Review, 79(1), 145.

[58] Pons J. M. Ostrogradski's Theorem for Higher-Order Singular Lagrangians, Lett. Math. Phys. 17 (1989) 181189.

[59] Prieto-Martínez, P. D., and Román-Roy, N. (2011). Lagrangian-Hamiltonian unified formalism for autonomous higher order dynamical systems. Journal of Physics A: Mathematical and Theoretical, 44(38), 385203.

[60] Prieto-Martínez, P. D., and Román-Roy, N. (2012). Unified formalism for higher order non-autonomous dynamical systems. Journal of Mathematical Physics, 53(3), 032901.

[61] H. Rund, Canonical formalism for parameter-invariant integrals in the calculus of variations whose Lagrange functions involve second-order derivatives, Ann. Mat. Pura Appl. 63 (1964) 99-107.

[62] Rashid, M. S., and Khalil, S. S. (1996). Hamiltonian description of higher order lagrangians. International Journal of Modern Physics A, 11(25), 4551-4559.

[63] Shadwick, Lett. Math. Phys. 6 (1982) 409-416.

[64] Sarığlu, Ö., and Tekin, B. (2006). Topologically massive gravity as a Pais Uhlenbeck oscillator. Classical and Quantum Gravity, 23(24), 7541.

[65] Saunders, J. Phys. A 20 (1987) 339-349.

[66] Saito, y., Sugano, R., Ohta, T., and Kimura, T. (1989). A dynamical formalism of singular Lagrangian system with higher derivatives. Journal of mathematical physics, 30(5), 1122-1132.

[67] Schmidt, H. J. (1994). Stability and Hamiltonian formulation of higher derivative theories. Physical Review D, 49(12), 6354.

[68] Schmidt, H. J. (1995). An alternate Hamiltonian formulation of fourth-order theories and its application to cosmology. arxiv preprint gr-qc/9501019. 
[69] Skinner, R. (1983). First order equations of motion for classical mechanics. Journal of mathematical physics, 24(11), 2581-2588.

[70] Skinner, R., and Rusk, R. (1983). Generalized Hamiltonian dynamics. I. Formulation on $T^{*} Q \oplus T Q$. Journal of mathematical physics, 24(11), 2589-2594..

[71] Skinner, R., and Rusk, R. (1983). Generalized Hamiltonian dynamics. II. Gauge transformations. Journal of mathematical physics, 24(11), 2595-2601.

[72] E. C. G. Sudarshan and N. Mukunda, Classical Dynamics: A Modern Perspective (Wiley, New york, 1974);

[73] Suri, A. (2017). Higher Order Tangent Bundles. Mediterr. J. Math. 14 (2017), 14:5.

[74] Urban, Z., and Krupka, D. (2013). The Zermelo conditions and higher order homogeneous functions. Publicationes Mathematicae-Debrecen, 82(1), 59-76.

[75] Vitagliano, L. (2010). The Lagrangian-Hamiltonian formalism for higher order field theories. Journal of Geometry and Physics, 60(6), 857-873.

[76] E. Zermelo, Untersuchungen zur Variationsrechnung, Dissertation, Friedrich-Wilhelms-Universit“at, Berlin, 1894.

[77] Tulczyjew, W. M. (1977). The legendre transformation. In Annales de l'IHP Physique thÃ (corique (Vol. 27, No. 1, pp. 101-114). 